\title{
WestVirginiaUniversity
}

THE RESEARCH REPOSITORY @ WVU

Graduate Theses, Dissertations, and Problem Reports

1998

\section{The Prevalence of Posttraumatic Stress Disorder among Federal Prison Inmates}

Robert K. Guthrie

West Virginia University

Follow this and additional works at: https://researchrepository.wvu.edu/etd

Part of the Counseling Psychology Commons

\section{Recommended Citation}

Guthrie, Robert K., "The Prevalence of Posttraumatic Stress Disorder among Federal Prison Inmates" (1998). Graduate Theses, Dissertations, and Problem Reports. 3951.

https://researchrepository.wvu.edu/etd/3951

This Dissertation is protected by copyright and/or related rights. It has been brought to you by the The Research Repository @ WVU with permission from the rights-holder(s). You are free to use this Dissertation in any way that is permitted by the copyright and related rights legislation that applies to your use. For other uses you must obtain permission from the rights-holder(s) directly, unless additional rights are indicated by a Creative Commons license in the record and/ or on the work itself. This Dissertation has been accepted for inclusion in WVU Graduate Theses, Dissertations, and Problem Reports collection by an authorized administrator of The Research Repository @ WVU.

For more information, please contact researchrepository@mail.wvu.edu. 


\section{INFORMATION TO USERS}

This manuscript has been reproduced from the microfilm master. UMI films the text directly from the original or copy submitted. Thus, some thesis and dissertation copies are in typewriter face, while others may be from any type of computer printer.

The quality of this reproduction is dependent upon the quality of the copy submitted. Broken or indistinct print, colored or poor quality illustrations and photographs, print bleedthrough, substandard margins, and improper alignment can adversely affect reproduction.

In the unlikely event that the author did not send UMI a complete manuscript and there are missing pages, these will be noted. Also, if unauthorized copyright material had to be removed, a note will indicate the deletion.

Oversize materials (e.g., maps, drawings, charts) are reproduced by sectioning the original, beginning at the upper left-hand comer and continuing from left to right in equal sections with small overlaps. Each original is also photographed in one exposure and is included in reduced form at the back of the book.

Photographs included in the original manuscript have been reproduced xerographically in this copy. Higher quality 6" $\times 9$ " black and white photographic prints are available for any photographs or illustrations appearing in this copy for an additional charge. Contact UMI directly to order.

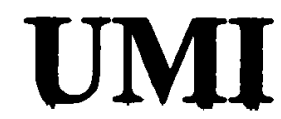

A Bell \& Howell Information Company 300 North Zeeb Road, Ann Astor MI 48106-1346 USA

$313 / 761-4700 \quad 800 / 521-0600$ 
Reproduced with permission of the copyright owner. Further reproduction prohibited without permission. 
THE PREVALENCE OF

POSTTRAUMATIC STRESS DISORDER

AMONG FEDERAL PRISON INMATES

By

Robert K. Guthrie

\author{
A Dissertation \\ submitted to \\ The College of Human Resources and Education \\ at \\ West Virginia University \\ in partial fulfillment of the requirements \\ for the degree of \\ Doctor of Philosophy \\ in \\ Counseling Psychology
}

Department of Counseling, Rehabilitation Counseling, and Counseling Psychology

David J. Srebalus, Chair

Morgantown, West Virginia

1998 
UXI Number: 9926661

\section{Copyright 1998 by \\ Guthrie, Robert Rarl}

All rights reserved.

UMI Microform 9926661

Copyright 1999, by UMI Company. All rights reserved.

This microform edition is protected against unauthorized copying under Title 17, United States Code. 
(C) 1998

\section{Robert Karl Guthrie}




\section{Dedication}

This dissertation is dedicated to Helen English Guthrie, my editor and the love of my life. 


\section{Acknowledgments}

The author expresses his gratitude to West Virginia University for its generous support of his doctoral studies via the Arlen and Louise Stone Swiger Doctoral Fellowsinip; to the United States Department of Justice, Federal Bureau of Prisons, for permitting the research to take place; to the staffs of the Federal Correctional Institution and Federal Prison Camp, Cumberland, Maryland, for their assistance in carrying out the research; to the Chair of his Dissertation Committee, Dr. David J. Srebalus, Professor, Department of Counseling, Rehabilitation Counseling, and Counseling Psychology, West Virginia University; to the members of that committee, Dr. Cynthia R. Kalodner, Assistant Professor, Department of Counseling, Rehabilitation Counseling, and Counseling Psychology, West Virginia University, Dr. Lisa L. Morshead, Chief Psychologist, Federal Correctional Institution, Cumberland, MD, Dr. Joseph R. Scotti, Assistant Professor, Department of Clinical Psychology, West Virginia University, and Dr. Richard T. Walls, Professor, Research and Training Center, West Virginia University. Finally, sincere thanks is due the 100 men who agreed to participate in this study for the benefit of others. 


\section{Contents}

Chapter 1: An Overview of the Problem

Introduction

The Problem

Incarceration statistics

Mental disorder among inmates

PTSD Prevalence and Assessment

Estimates of PTSD in the general population

PTSD prevalence among at-risk groups

Standardized assessment and PTSD prevalence 3

The Impact of PTSD $\quad 4$

Sequelae and comorbid disorders 4

Treatment of Mental Ilness Among Prison Inmates 5

Treatment rights and realities 5

Additional reasons to treat $\quad 6$

Mental illness, crime, and violence $\quad 6$

$\begin{array}{ll}\text { Purposes of the Study } & 7\end{array}$

Primary Purposes 7

Secondary Purposes $\quad 7$

$\begin{array}{ll}\text { Hypotheses } & 8\end{array}$

Assessment Instruments $\quad 8$

Definition of Key Terms

Chapter 2: Literature Review

$\begin{array}{ll}\text { Introduction } & 13\end{array}$

$\begin{array}{ll}\text { Baseline Data } & 14\end{array}$

$\begin{array}{ll}\text { Prevalence } & 14\end{array}$

$\begin{array}{ll}\text { Using DSM-III criteria } & 14\end{array}$

Using DSM-III-R criteria $\quad 14$

Disorders Reported as Comorbid with PTSD 18

$\begin{array}{ll}\text { Summary } & 20\end{array}$ 
$\begin{array}{ll}\text { Exposure Data } & 20\end{array}$

Vulnerability $\quad 20$

The Nature of Traumatic Stressors 21

General information $\quad 21$

Motor vehicle accidents $\quad 22$

Injuries and assaults 23

Occupations and PTSD 23

Homelessness $\quad 23$

Substance abuse and PTSD $\quad 24$

PTSD and physical illness $\quad 24$

PTSD among veterans $\quad 24$

PTSD and natural disasters $\quad 25$

Conclusions $\quad 25$

Mental Disorder and Prison Inmates $\quad 26$

Mental Disorder and Criminality 26

The general relation $\quad 26$

Longitudinal and birth cohort studies $\quad 27$

The Prevalence of Mental Disorders Among Inmates 27

Co-occurring Mental Disorders Among Inmates 31

The Prevalence of PTSD Among Inmates 32

Mental Disorder, Prison Inmates, and Society 33

Why Provide Prison Mental Health Services? 33

Danger to self and others 33

Benefit from prison activities $\quad 35$

Need to be transferred $\quad 35$

Capacity for treatment 36

Conclusion $\quad 36$

Identification of Mental Disorders 36

Among Individuals $\quad 36$

Among Groups 37 
Summary

Chapter 3: Method

Introduction

Participants

Power Analysis

Materials

Clinician-Administered PTSD Scale (CAPS-DX) 45

Development 45

Administration and scoring $\quad 46$

$\begin{array}{ll}\text { Psychometric characteristics } & 47\end{array}$

Keane PTSD Scale of the MMPI-2 (PKS) 47

$\begin{array}{ll}\text { Development } & 47\end{array}$

$\begin{array}{ll}\text { Administration and scoring } & 48\end{array}$

$\begin{array}{ll}\text { Psychometric characteristics } & 49\end{array}$

Posttraumatic Stress Diagnostic Scale ${ }^{\text {TM }}$ (PDSTM) 50

Development $\quad 50$

Administration and scoring $\quad 51$

Psychometric characteristics $\quad 53$

Hollingshead Four-Factor Index of Social Status (HFFI) 53

Development 53

Administration and scoring $\quad 53$

Psychometric characteristics $\quad 55$

Wechsler Adult Intelligence Scale - Revised (WAIS-R) 55

Development 55

Administration and scoring $\quad 56$

$\begin{array}{ll}\text { Psychometric characteristics } & 56\end{array}$

Chapter 4: Results

Introduction

The Research Questions $\quad 59$

$\begin{array}{lr}\text { Chapter Layout } & 59\end{array}$ 
The Research Setting $\quad 60$

$\begin{array}{ll}\text { Data collection sites } & 60\end{array}$

$\begin{array}{ll}\text { The Research Sample } & 60\end{array}$

Derivation and Characteristics $\quad 60$

$\begin{array}{ll}\text { Inmate populations } & 60\end{array}$

How the sample was determined 63

Characteristics of the sample $\quad 63$

Results 66

Potentially Traumatic Stressors Experienced by the Sample 66

Potentially traumatic stressors at the research sites 66

Number of potentially traumatic stressors experienced by

participants 66

Type of traumatic stressors identified to satisfy Criterion A $\quad 66$

Criterion A stressors identified by inmates with lifetime PTSD $\quad 70$

Criterion A stressors identified by inmates with current PTSD $\quad 70$

Percentage of stressors associated with lifetime and current PTSD 70

Frequency of lifetime and current stressors by race $\quad 70$

$\begin{array}{ll}\text { Prevalence } & 75\end{array}$

CAPS-DX diagnoses using standard scoring $\quad 75$

CAPS-DX diagnoses using the Rule of Four 75

Distribution of PTSD-positive cases throughout the sample $\quad 75$

Comparison of CAPS-DX results with those of other diagnostic instruments 75

$\begin{array}{ll}\text { PTSD Symptoms } & 78\end{array}$

Mean number of symptoms experienced 78

CAPS-DX symptom scores $\quad 79$

Multiple Linear Regression Analysis $\quad 79$

The choice of a target variable 79

Selection of independent variables $\quad 80$

Regression results $\quad 80$ 
Analysis of the Study's Hypotheses $\quad 84$

Hypothesis $1 \quad 84$

Hypothesis $2 \quad 85$

Hypothesis $3 \quad 85$

Hypothesis $4 \quad 86$

Hypothesis $5 \quad 86$

Hypothesis $6 \quad 86$

$\begin{array}{ll}\text { Hypothesis } 7 & 87\end{array}$

Summary of Hypothesis Support $\quad 87$

Chapter 5: Discussion

$\begin{array}{ll}\text { Introduction } & 88\end{array}$

$\begin{array}{ll}\text { Study Findings } & 88\end{array}$

Primary Hypotheses $\quad 88$

Lifetime and current prevalence $\quad 88$

Prevalence by security level $\quad 89$

Awareness of corrections officials of the presence of PTSD 92

Secondary Hypotheses 93

IQ and SES differences for inmates with and without PTSD 93

Racial differences for inmates with and without PTSD 94

Incarceration and other events as PTSD stressors 97

Type I and Type II trauma $\quad 100$

Effectiveness of Diagnostic Instruments 101

Keane Scale (PKS) 101

Posttraumatic Stress Diagnostic Scale ${ }^{\mathrm{TM}}$ (PDS ${ }^{\mathrm{TM}}$ ) 102

Recommendations for correctional use 102

Group treatment 103

Study Contributions, Limitations, and Future Directions 103

$\begin{array}{ll}\text { Study Contributions } & 103\end{array}$ 
$\begin{array}{ll}\text { Study Limitations } & 104\end{array}$

$\begin{array}{ll}\text { The site } & 104\end{array}$

$\begin{array}{ll}\text { Overall participation } & 104\end{array}$

$\begin{array}{ll}\text { The sample } & 104\end{array}$

Use of a single researcher 105

How limitations could be improved 106

$\begin{array}{ll}\text { Future Directions } & 106\end{array}$

$\begin{array}{ll}\text { A larger sample size } & 106\end{array}$

Varied racial composition of sample sites $\quad 106$

$\begin{array}{ll}\text { A varied research team } & 106\end{array}$

$\begin{array}{ll}\text { The importance of multiple stressors } & 107\end{array}$

The presence of complex PTSD symptomatology 107

$\begin{array}{ll}\text { Summary } & 107\end{array}$

$\begin{array}{ll}\text { References } & 109\end{array}$

$\begin{array}{ll}\text { Appendix A: Diagnostic Criteria } & 122\end{array}$

Appendix B: Research Questionnaire 125

$\begin{array}{ll}\text { Abstract } & 127\end{array}$

$\begin{array}{lr}\text { Curriculum Vitae } & 129\end{array}$ 
Tables

Table 1: General Population Estimates of the Prevalence of PTSD 16

Table 2: PTSD Prevalence Ranges for Males, Females, and General Population 17

Table 3: Odds Ratios for Disorders Comorbid with PTSD 19

Table 4: Prevalence Rates of Mental Disorders for U. S. and Canadian Inmates 29

Table 5: PDSTM Severity Levels 52

Table 6: PDS ${ }^{\mathrm{TM}}$ Estimated Level of Impairment 52

Table 7: Hollingshead's (1975) Educational Scores 54

Table 8: Hollingshead's Social Stratum and Score Range on the HFFI 54

Table 9: Interpretation of WAIS-R IQ Levels 57

Table 10: Racial Composition: Federal Prison System, Sample, and FPC and FCI Cumberland 61

Table 11: Chi-Square Comparisons of Black and White Populations 61

Table 12: Sample Recruitment: Distribution of Participants and Non-participants 62

Table 13: Age, IQ, and SES Means by Security Level 65

Table 14: Age, IQ, and SES Means for Blacks and Whites 65

Table 15: Age, IQ, and SES Means for Inmates with and without PTSD 65

Table 16: Mean Number of Potential Stressors Identified by $\begin{array}{ll}\text { CAPS-DX and PDSTM } & 67\end{array}$

Table 17: Stressors Identified to Satisfy Criterion A: Totals by Sample, Security Level, and Race 68

Table 18: Stressors Associated with Lifetime PTSD: Totals by Sample, Security Level, and Race

Table 19: Stressors Associated with Current PTSD: Totals by Sample, Security Level, and Race $\quad 71$

Table 20: Percentage of Stressor Category Resulting in Lifetime PTSD 72

Table 21: Percentage of Stressor Category Resulting in Current PTSD 73

Table 22: Racial Distribution of Stressors for Lifetime and Current PTSD Diagnoses $\quad 74$

Table 23: Lifetime and Current PTSD Diagnoses by Security Level 76 
Table 24: Lifetime and Current PTSD Diagnoses by Race

Table 25: Mean Instrument Scores for Lifetime PTSD Diagnosis

Table 26: Means and Standard Deviations for Regression Variables

Table 27: Bivariate Correlations

82

Table 28: Multiple Linear Regression Results

83

Table 29: Lifetime Prevalence Rates by Group

90 


\section{Chapter 1: AN OVERVIEW OF THE PROBLEM}

Introduction

The Problem

Incarceration statistics. Incarceration in the United States has increased dramatically in recent years. The total number of inmates under the jurisdiction of the Federal Bureau of Prisons (BOP) on September 30, 1995, was 100,958, an increase of over 11,000 (12\%) from January 1, 1994 (U. S. Department of Justice, 1995a) and an increase of over 60,000 (152\%) from 1981 (U. S. Department of Justice, 1994a). Approximately $92 \%$ of this population is male. Rubin and McCampbell (1995) point out that conservative estimates indicate that $6-8 \%(6,000-8,000)$ of inmates currently under BOP control exhibit a major mental disorder, such as bipolar affective disorder, major depression, or schizophrenia.

Mental disorders among inmates. Efforts have been made to provide specific estimates of the prevalence of Diagnostic and Statistical Manual (DSM) Axis-I disorders among inmates (Daniel, Robins, Reid, \& Wilfrey, 1988; Teplin, 1990b). What these studies have shown is that anxiety (e.g., generalized anxiety, phobias, posttraumatic stress) and mood (e.g., major depression, dysthymia) disorders are the most prevalent among inmates, followed by substance abuse disorders (Hodgins \& Cote. 1990). For postrraumatic stress disorder (PTSD), in particular, lifetime prevalence rates have ranged from 2.3-6\% of male inmates in three U. S. studies to $13 \%$ in a Canadian study (Collins \& Bailey, 1990; Hodgins \& Cote). The Canadian study (Hodgins \& Cote) was the only one to report a current prevalence rate of PTSD among inmates. The percentage of individuals who were PTSD-positive within six months of the clinical interview was 3.9\%. As will be seen, when these results are compared with methodologically similar studies that estimate mental disorders in the general population (Breslau, Davis, Andreski, \& Peterson, 1991; Davidson, Hughes, Blazer, \& George, 1991; Helzer, Robins, \& McEvoy, 1987; Kessler, Sonnega, Bromet, Hughes, \& Nelson, 1995) it is clear that mental disorders, including PTSD, are much more prevalent among prison inmates than among the general citizenry. 


\section{PTSD Prevalence and Assessment}

Estimates of PTSD in the general population. At first, researchers believed that PTSD was a rather rare occurrence. The earliest diagnostic and statistical manual to include PTSD, DSM-III, required the identification of a stressor that "would evoke significant symptoms of distress in almost everyone" (American Psychiatric Association, 1980 , p. 238). This rather vague and highly exclusionary definition was used in National Institute of Mental Health (NIMH) Epidemiologic Catchment Area (ECA) studies, which identified the lifetime rate of PTSD in the general population as ranging between 0.91.3\% (Davidson et al., 1991; Helzer et al., 1987). Only the Davidson et al. study identified a six-month prevalence rate, which was $0.44 \%$.

These studies used an instrument called the Diagnostic Interview Schedule (DIS) for the DSM-III to identify mental disorders. The DIS has been shown to lack diagnostic sensitivity (Kulka et al., 1991), and, therefore, studies that rely on it for diagnoses are believed to underreport prevalence rates. This same instrument was used in most studies of PTSD prevalence among prison inmates (Hodgins \& Cote, 1990). Thus, it is possible that these studies have also underreported prevalence rates.

DSM-III-R brought more precision to the definition of PTSD, and the precipitating stressor became an event "outside the range of usual human experience...that would be markedly distressing to almost anyone" (American Psychiatric Association, 1987, p. 146). The definition then went on to identify a number of stressors that could precipitate PTSD, such as "serious threat to one's life or physical integrity; serious threat or harm to one's children, spouse or other close relatives and friends; sudden destruction of one's home or community;" etc. (American Psychiatric Association, 1987, p. 146). This change in the diagnostic criteria, which was reflected in the new DIS for the DSMIII-R, may have resulted in different estimates of the prevalence of PTSD within the general population. Whereas lifetime prevalence using the old DIS for the DSM-III (1980) had been estimated at approximately $1 \%$, use of the new DIS for the DSM-III-R resulted in a rate of approximately $8 \%$. In these new studies, males evidenced a rate of 5 $6 \%$ and females one of 10-11\% (Breslau et al., 1991; Kessler et al., 1995). Kessler et al. 
further noted that for approximately one-third of these individuals the PTSD had become chronic.

PTSD prevalence among at risk groups. A large number of studies have also been conducted indicating the prevalence rate for PTSD is even higher among groups of individuals who are "at risk" due to their exposure to a variety of stressors. McFarlane and de Girolamo (1996) surveyed a wide range of studies that assessed many of these groups, including fire fighters; survivors of natural disasters such as floods, hurricanes, and volcanic eruptions; survivors of sexual and physical abuse; victims of man-made accidents (motor vehicles, trains); individuals exposed to concentration camps; political prisoners; victims of torture; and military personnel, especially those exposed to combat. McFarlane and de Girolamo found that PTSD prevalence rates for at-risk groups can range from $20-80 \%$. Therefore, a substantial body of information indicates that individuals exposed to a variety of stressors are at risk to develop PTSD. As methodologically comparable epidemiological studies have consistently indicated that inmates have prevalence rates of PTSD greater than those of the general public (Hodgins \& Cote, 1990) it seems reasonable to hypothesize that inmates are more "at risk" of having been exposed to potentially traumatic events than members of the general population.

Standardized assessment and PTSD prevalence. To identify a PTSD prevalence rate for inmates in which prison administrators and psychologists can have confidence, researchers need to use assessment instruments of demonstrated sensitivity (Hodgins, 1995; Roesch, Ogloff, \& Eaves, 1995). As we have already seen, changes in the DSM PTSD criteria and the DIS seem to have had an impact on prevalence rates obtained in different studies. Kulka et al. (1991) helped bring this problem into sharper focus by comparing two major studies involving PTSD prevalence rates among Vietnam veterans: The Centers for Disease Control (CDC) Vietnam Experience Study (VES) and the National Vietnam Veterans Readjustment Survey (NVVRS). These two studies arrived at very different PTSD prevalence rates for Vietnam vets. The CDC VES (which used the DIS as it applied to DSM-III (1980) identified a prevalence rate among veterans closer to that of the ECA studies previously discussed--about $2.2 \%$. However, the NVVRS, which 
used multiple diagnostic measures, including the Structured Clinical Interview for DSMIII-R (SCID), identified a PTSD prevalence rate among Vietnam veterans of approximately $15 \%$. When the studies were compared by Kulka et al., the difference in the prevalence rates was shown to have been a direct result of the different measures used.

Subsequent evaluations of the SCID have indicated that it is four to five times more sensitive than the DIS, leading researchers to believe that studies which used the DIS substantially underestimated the prevalence of PTSD (Newman, Kaloupek, \& Keane, 1996). But Newman et al. (1996) also note that the SCID may overestimate lifetime prevalence due to the fact that symptoms are identified on a "worst ever" basis.

Therefore, endorsed symptoms may not have occurred at the same time during a person's life.

The study of male prison inmates in Canada cited earlier (Hodgins \& Cote, 1990) used the DIS for the DSM-III-R and identified a PTSD prevalence rate of $13 \%$. Although this may seem contradictory, one of the reasons given for this much higher rate (Hodgins, 1995) was the fact that the researchers were able to guarantee their participants absolute confidentiality. It also seems possible the change from DSM-III (1980) to DSM-III-R (1987), and the concomitant change in the DIS, contributed to these different findings.

All of this appears to indicate how likely it is that epidemiological studies of prison inmates have also underestimated the prevalence of the disorder among this population and that the issue needs to be revisited.

\section{The Impact of PTSD}

Sequelae and comorbid disorders. Since its first formal recognition in DSM-III (1980) as a psychiatric disorder, PTSD has enjoyed increased attention and this has led to a number of conclusions about the effects of trauma on humans. In general, the disorder now appears to be linked to somatization, affective dysregulation, and dissociation (van der Kolk et al., 1996). In addition, the type of trauma that leads to PTSD has been demonstrated to bring about physical changes in the brain and disruption of neurotransmitter homeostasis (van der Kolk, 1996a). PTSD is seldom found in its pure form, and tends to co-occur with a variety of other psychiatric disorders, which at least 
one researcher (van der Kolk, 1995) views as mechanisms for coping with the original trauma. These comorbid disorders can include general anxiety disorder, panic disorder, obsessive-compulsive disorder, dissociative disorders (including dissociative identity disorder), major depression, dysthymia, substance abuse and dependence, and antisocial personality disorder (APD) (Kessler et al., 1995; Kulka et al., 1991).

Therefore, the presence of PTSD is not solely a problem in and of itself, but may bring with it a variety of other psychiatric disorders that may have an impact on the inmate's current and future functioning and on his ability to participate in and benefit from programs designed to assist him. For example, a high percentage of BOP inmates have been incarcerated for offenses involving drugs and/or alcohol (U. S. Department of Justice, 1995a). In minimum and low security facilities, drugs and/or alcohol have been a problem for approximately $67 \%$ of all inmates, while at the medium security level this number is approximately $57 \%$ of all inmates (U. S. Department of Justice, 1995a). According to van der Kolk (1995), it appears as though drugs and alcohol may be used by individuals with PTSD as a way of regulating their affect. If this is the case, then it raises interesting questions about one of the major rehabilitation programs conducted by the BOP: A biopsychosocial program aimed at reducing inmate drug and alcohol dependence and abuse. If that dependence and/or abuse is the result of or comorbid with untreated PTSD, then the program may be less effective than hoped, unless the PTSD is identified and also treated concurrent with the drug and/or alcohol problem.

\section{Treatment of Mental Dlness among Prison Inmates}

Treatment rights and realities. By law (Dvoskin \& Steadman, 1989; Rubin \& McCampbell, 1995) inmates are entitled to treatment for their mental disorders, but it stands to reason that such treatment is only possible after an appropriate diagnosis has been made. Unfortunately, however, inmate populations are not always adequately screened for mental disorders. Teplin (1990a) has shown that in a county jail the inmates most likely to receive treatment for psychiatric disorders are those with previous mental health system contact which has become part of their prison record. Even inmates with major mental disorders, such as schizophrenia, are often ignored if the disorder has not been identified in their prison record. Therefore, it appears as though improved 
identification of inmates with mental disorders might assist in the effective allocation of limited resources.

Additional reasons to treat. In addition to the mandated need to treat, Ogloff, Roesch and Hart (1993) cite four reasons why it is important for prison administrators to be aware of an inmate's mental health status: (a) to determine if the inmate poses a risk to self and/or others, (b) to determine an inmate's ability to participate in institutional activities, (c) to assess the need for transfer to a more appropriate facility, and (d) to determine if an inmate can benefit from treatment. It has been demonstrated that PTSD may be an important factor in each of these instances (Collins \& Bailey, 1990; Hodgins \& Cote, 1993; Kessler et al., 1995; Kulka et al., 1991; van der Kolk \& McFarlane, 1996; van der Kolk, McFarlane, \& van der Hart, 1996). Therefore, given that the identification of PTSD can be an important decision-making element for prison administrators and psychologists, it seems reasonable that an accurate determination of its prevalence among various segments of the inmate population would be helpful.

Mental illness, crime, and violence. Hodgins (1993) cites a number of studies indicating there is not only a general link between mental illness and crime, but between mental illness and violence, as well. Studies conducted by Kulka et al. (1991) and Collins and Bailey (1990) have demonstrated that positive correlations between PTSD and crime and between PTSD and violence also exist, and that the PTSD often predates the problematic behavior. Hodge (1992) has even proposed that PTSD may result in an "addiction to violence" (p. 212). All of this work provides a strong indication that addressing mental disorders, in general, and PTSD, in particular, may ultimately have an impact on both crime and violence. As Federal inmates in medium security facilities have committed more violent crimes than those in lower security facilities (U. S. Department of Justice, 1995a), it would be interesting to determine whether or not either of these groups manifests a higher prevalence of PTSD than the other. Based on the identified relation between PTSD and violence, it seems possible that medium security inmates will evidence higher rates of PTSD than minimum or low security inmates. 


\section{Purposes of The Study}

\section{Primary Purposes}

The primary purposes of this study include examining (a) whether or not existing estimates of the lifetime and current prevalence of PTSD among male inmates are reliable, (b) whether or not there is a correlation between prison security levels and the prevalence of PTSD, and (c) how aware prison authorities are of the existence of PTSD among the inmates under their control. To achieve these purposes, a total of 100 inmates, 50 each at a BOP minimum security facility and a BOP medium security facility, will be assessed for PTSD.

\section{Secondary Purposes}

The secondary purposes of this study involve an attempt to identify inmate groups that may evidence higher rates of PTSD due to personal or cultural characteristics. The identification of such groups might permit limited resources to be used most efficiently to identify individual inmates with PTSD. For example, Federal prison inmates tend to be over-representative of minorities (U. S. Department of Justice, 1995b) and, by implication, of lower socioeconomic groups. Kulka et al. (1991) have demonstrated that African- American and Hispanic male Vietnam veterans manifest a higher rate of PTSD than Caucasian Vietnam veterans. In addition, Shalev (1996) has shown that the lower an individual's socioeconomic status and education level, the more likely they are to experience subsequent exposure to stressors that can precipitate PTSD. The median educational level for state inmates (who make up over $90 \%$ of all prison inmates) is the 11 th grade (U. S. Department of Justice, 1994b). Given this, it would be interesting to see if African-American and Hispanic inmates, inmates who grew up in families of lower socioeconomic status (SES), or inmates with less formal education or lower IQs evidence higher rates of PTSD than Caucasian inmates, inmates from higher SES families, or inmates with more education or higher IQs. Finally, in a sample of 248 non-incarcerated subjects, Foa (1995) found that $14 \%$ listed imprisonment as a stressful event they had experienced. However, no one identified it as a direct precipitant of PTSD symptoms. It would be of interest to investigate whether or not incarceration is identified as a precipitant of PTSD by individuals currently in prison. 


\section{Hypotheses}

This study will be guided by the following primary $(\mathrm{Hl}-\mathrm{H} 3)$ and secondary $(\mathrm{H} 4$ H7) hypotheses:

H1. That the current and lifetime rates of PTSD among male inmates will be higher than those exhibited in the general population and higher than those found in previous estimates involving American inmates.

H2. That inmates incarcerated in a medium security facility will manifest a higher prevalence of PTSD than those in a minimum security facility.

H3. That the majority of inmates who are identified as PTSD-positive will not have been previously identified as such by corrections officials.

H4. That PTSD-positive inmates will have lower full scale intelligence quotient (IQ) estimates than inmates without PTSD.

H5. That the childhood socioeconomic status of PTSD-positive inmates will be lower than that of inmates without PTSD.

H6. That African-American and Hispanic inmates will have higher PTSD prevalence rates than Caucasian inmates.

H7. That, for some inmates, incarceration itself will have been a traumatic stressor.

\section{Assessment Instruments}

A debate has been conducted in the literature concerning the use of single vs. multiple assessment instruments, as well as the trade-off between diagnostic precision and the cost of assessment (Hodgins, 1995; Kulka et al., 1991). This study will use multiple assessment instruments with the intent of providing corrections officials with a variety of diagnostic options. The instruments to be used for the assessment of PTSD are fully described in Chapter 3 of this document and will include: the ClinicianAdministered PTSD Scale-DX (CAPS-DX) developed by Blake et al. (1990); the Minnesota Multiphasic Personality Inventory-2 (MMPI-2) P-K scale for the assessment of PTSD (PKS) developed by Keane, Malloy, and Fairbank (1984); and the Posttraumatic Stress Diagnostic Scale ${ }^{\mathrm{TM}}$ (PDS ${ }^{\mathrm{TM}}$ ) (Foa, 1995). In addition, a questionnaire will be used to gather demographic information, including data related to socioeconomic status and 
substance abuse/dependence. Selected subtests from the Wechsler Adult Intelligence Scale, Revised (WAIS-R) (Wechsler, 1981) will be administered to determine global IQ. Information concerning identification of PTSD by corrections psychologists will be obtained through review of inmate psychology files.

\section{Definition of Key Terms}

At risk: In most studies the term "at risk" refers to individuals who carry a genetic predisposition to develop a particular disease. Within the context of this study "at risk" refers to individuals who have been exposed to the traumatic stressors that are thought to precipitate PTSD.

Clinician-Administered PTSD Scale-DX (CAPS-DX): A structured interview designed to assess the existence of PTSD symptoms according to DSM-IV (1994) diagnostic criteria and to address many of the deficiencies of other PTSD interviews (e.g., the DIS and SCID). Its major drawback is its administration time, which can be up to one hour. However, the CAPS-DX allows the clinician to assess for both lifetime and current PTSD, as well as the severity of the disorder. The CAPS-DX has good specificity (i.e., it generates few false positives) and good sensitivity (i.e., it does a good job of identifying all those who actually qualify for the PTSD diagnosis). The CAPS-DX includes a Life Events Checklist that permits the identification of multiple stressors.

Comorbidity: The co-existence of psychiatric disorders, the likelihood of which is determined by an odds ratio. Disorders such as general anxiety disorder, panic disorder, dissociative disorder, obsessive-compulsive disorder, major depression, dysthymia, antisocial personality disorder and alcohol and/or drug abuse or dependence are often comorbid with PTSD.

Current prevalence: The percentage of individuals who were identified as PTSD-positive at the time of assessment. Although current prevalence is subsumed under lifetime prevalence, not all lifetime cases of PTSD are currently active.

Diagnostic Interview Schedule (DIS): The DIS is a semistructured interview that has been used in a number of community and disaster studies to estimate the prevalence of a variety of psychiatric disorders, including PTSD. However, research has indicated that while the DIS has good specificity, it has poor sensitivity. Advantages of using the PTSD 
module of the DIS include its administration time (approximately 15 minutes); the fact that it can be administered by a layperson; and the fact that it has been used for a number of epidemiological studies, thus ensuring a measure of consistency.

Epidemiology: Stein and Susser (1981) describe epidemiology as "the science concerned with the health of populations or communities. The compass of this science is to describe states of health and their variation, to discover the determinants of the variations observed, and to use what is discovered to devise and test ways of preventing and controlling ill health" (p. 444).

Incidence: Stein and Susser (1981) state that incidence is the frequency of a disorder's occurrence through time. Incidence can "refer to all episodes whenever they occur and not just to inceptions (first episodes)" (p. 445).

Lifetime prevalence: Refers to the existence of PTSD at any given point during an individual's life. A participant who has had PTSD during his or her lifetime may not currently be PTSD-positive.

Partial Posttraumatic Stress Disorder: Various definitions of partial PTSD, which is also known as "subsyndromal" or "subclinical" PTSD, exist. Blanchard, Hickling, Taylor, Loos, and Gerardi (1994) used this term to refer to individuals who met the DSM-III-R (1987) criteria A and B fully, plus either the avoidance symptom criterion (C) or the overarousal symptom criterion. Green, Epstein, Krupnick, and Rowland (1997) tightened this somewhat by requiring the individual to meet DSM-IV (American Psychiatric Association, 1994) criteria A, B, and C fully, while experiencing only one of two required criterion D symptoms. As Foa, Riggs, and Gershuny (1995) have identified numbing as a symptom whose presence or absence can distinguish between individuals with and without PTSD, this study will utilize the Green et al. (1997) criteria, which require that the necessary three avoidance and/or numbing symptoms of cluster $C$ be endorsed. Posttraumatic Stress Disorder: The DSM-IV (1994) states that to be diagnosed with posttraumatic stress disorder (PTSD), an individual "must have been exposed to a traumatic event in which both of the following were present: (1) the person experienced, witnessed, or was confronted with an event or events that involved actual or threatened death or serious injury, or a threat to the physical integrity of self or others, and (2) the 
person's response involved intense fear, helplessness, or horror" (pp. 427-428). In addition, the event must be "persistently reexperienced," the individual must experience "persistent avoidance of stimuli associated with the experience and numbing of general responsiveness," and "persistent symptoms of increased arousal" must be present (p. 428).

Prevalence: Stein and Susser (1981) state that prevalence "refers to cases of the health disorder present (prevalent) in a population at a given time of survey" (p. 446). This means that researchers are identifying the number of cases of a disorder that exist at a specific moment in time (i.e., today, past three months, past 12 months).

Sensitivity: The ability of an assessment instrument to identify actual cases or positives. Sensitivity ranges from 0 to $l$, with numbers closer to $l$ indicating greater sensitivity. Specificity: The ability of an assessment instrument to identify and exclude false positives. Specificity also ranges from 0 to 1 , with numbers closer to 1 indicating greater specificity. It is often the case that an increase in sensitivity will result in a decrease in specificity and vice versa.

Stressor: A stressor is any event that fulfills the necessary requirements cited in criterion A of the DSM definition of PTSD. For example, seeing someone shot, being shot, being in an automobile accident, being caught in a mine cave-in, being threatened by a hurricane, becoming a political prisoner, having a myocardial infarction, experiencing the death of a spouse or child, being raped, participating in combat, witnessing or participating in an assault or robbery, or being severely beaten might all serve as criterion A stressors. The development of symptomatology depends on a number of factors, including premorbid

functioning, dissociation at the time of the event (peritraumatic dissociation), the amount of stress that was experienced, the manner in which it was interpreted (i.e., as lifethreatening or not), and the number of potential stressors that preceded the precipitating event.

Structured Clinical Interview for the DSM-IV - PTSD Module (SCID): This is a semistructured clinical interview that has been used in a number of PTSD studies across diverse populations. It has good reliability and specificity, much better sensitivity than the 
DIS, and its results correlate well with other measures of PTSD, such as the PKS (Newman, Kaploupek, \& Keane, 1996). The SCID measures lifetime incidence as well as current and chronic prevalence. It takes somewhat longer than the DIS to administer (approximately 25 minutes), must be administered by a clinician rather than a trained technician, and may have a tendency to inflate lifetime rates due to its focus on "worst ever" symptomatology (Newman et al.). 


\section{Chapter 2: LITERATURE REVIEW}

\section{Introduction}

The incarceration of Americans is growing at a rapid pace. On December 31, 1994 , the total number of inmates in federal and state prisons was $1,053,738$. Ten years earlier, that number had been 462,002 , which is an increase of $128 \%$ (US Department of Justice, 1994a). The number of inmates under the control of the federal government alone increased 12\% from January 1, 1994 to September 30, 1995, to a total of 100,958. Approximately $92 \%$ of the federal prison population is male, $36.6 \%$ is African-American, and $27.2 \%$ is Hispanic (US Department of Justice, 1995a). The prisons in which these men are housed are at $125 \%$ of capacity (US Department of Justice, 1995b).

It has been reported by Rubin and McCampbell (1995) that conservative estimates of severe mental disability among inmates range from $6.4-8 \%$, but they do not define what they mean by "severe mental disability." Hodgins and Cote (1990) cite studies in the US and Canada that identify prevalence rates among inmates for schizophrenia of 1.2$7.5 \%$, for bipolar disorder of $1.1-4.8 \%$, and for major depression of 5.3-16.9\%. Furthermore, the prevalence of alcohol and drug abuse and/or dependence ranges from $21.8 \%$ to $46.2 \%$ in these studies. This means that tens of thousands of men in the federal prison system are experiencing one or more of these major problems. Dvoskin and Steadman (1989) write that the United States Supreme Court in Bowring v. Godwin (1977) held that services for mentally ill inmates are just as vital as other medical services, and Rubin and McCampbell (1995) state that according to the Americans with Disabilities Act, inmates cannot be denied treatment for mental illness.

It seems clear that corrections officials are faced with a substantial mental health challenge. However, due to a paucity of sound epidemiological data, the precise dimensions of the challenge remain poorly defined. Therefore, before one can even begin to address the problem effectively, the collection of accurate data concerning the prevalence of specific mental disorders is a necessity. This paper addresses that issue with regard to posttraumatic stress disorder (PTSD). 


\section{Baseline Data}

\section{Prevalence}

Using DSM-III criteria. In the 1980s, Epidemiological Catchment Area (ECA) studies were conducted by the National Institute of Mental Health (NMH) to provide data on the prevalence of mental disorders in the general population of the United States. The studies emphasized lifetime prevalence rates because lifetime prevalence of all disorders can be assessed via a single clinical interview. The use of a single instrument allows researchers to screen a larger population sample and, as a consequence, to identify more individuals who have had a certain disorder (Robins et al., 1984). These studies all used the same methodology, thus permitting comparison across sites.

The specific instrument used was the Diagnostic Interview Schedule (DIS). The DIS was developed at Washington University in St. Louis and provided "a set of questions to be read verbatim for each DSM-III (1980) criterion item for about 40 disorders and subtypes" (Helzer, Spitznagel, \& McEvoy, 1987, p. 1069). The DSM-III was the first DSM to include diagnostic criteria for posttraumatic stress disorder. The major, necessary criterion (Criterion $\mathrm{A}$ ) required for a diagnosis of PTSD using the DSMIII was the "existence of a recognizable stressor that would evoke significant symptoms of distress in almost everyone" (American Psychiatric Association, 1980, p. 238).

Researchers for two of the NIMH ECA studies provided information concerning the lifetime prevalence of posttraumatic stress disorder. Helzer et al. (1987) were the first to do so, and they identified a lifetime rate for PTSD of $1 \%$ among a sample of 2,493 participants in the St. Louis ECA study. However, this rate differed for men and women, being $0.5 \%$ for the former and $1.3 \%$ for the latter. Davidson et al. (1991) identified a lifetime rate for PTSD of $1.3 \%$ among 2,985 subjects who participated in the ECA conducted in the Piedmont region of North Carolina. However, they did not report a breakdown of their data by sex. Thus, these two early studies identified the rate of lifetime PTSD within the general population as $1-1.3 \%$.

Using DSM-III-R criteria. DSM-III-R (American Psychiatric Association, 1987) brought a revision of the diagnostic criteria for PTSD. One of the most important changes was the expansion of Criterion A from what was cited above to the following: "The 
person has experienced an event that is outside the range of usual human experience and that would be markedly distressing to almost anyone, e.g. serious threat to one's life or physical integrity; serious threat or harm to one's children, spouse, or other close relatives and friends; sudden destruction of one's home or community; or seeing another person who has recently been, or is being, seriously injured or killed as the result of an accident or physical violence" (p. 146). As Kessler et al. (1995) point out, research on PTSD stressors has indicated that they are rather common occurrences, well within the range of experience of most individuals. Despite this deficiency, the new definition did give more guidance to clinicians and was incorporated in the DIS for the DSM-III-R. This new DIS was used in two subsequent research studies that attempted to estimate the lifetime prevalence of PTSD within the general population.

The first of these studies was conducted by Breslau et al. (1991). These researchers studied 1007 young adults in Detroit, Michigan. The lifetime rate of PTSD identified in this study was $9.2 \%$, with women experiencing a lifetime rate of $11.3 \%$ and men a lifetime rate of $6 \%$. Kessler et al. (1995) administered the DIS for the DSM-III-R to a "representative national sample of 5,877 persons aged 15 to 54 years" (p. 1048). Their results indicated a lifetime prevalence for PTSD of $7.8 \%$, with females evidencing a rate of $10.4 \%$ and males a rate of $5 \%$. Table 1 provides a summary of these findings. The PTSD lifetime prevalence rates using the DIS for the DSM-III-R ranged from 7.8-9.2\% and were quite a bit higher than those achieved using the DIS for the DSM-III. Using the DIS for the DSM-III-R, the rates for females ranged from 10.4-11.3\%, while those for males ranged from 5-6\%. Table 2 provides a summary of these figures.

Therefore, it seems possible that the change in diagnostic criteria from DSM-III (1980) to DSM-III-R (1987) resulted in a change in the estimation of lifetime prevalence rates for the general population. However, as Kessler et al. (1995) state, "differences in diagnostic criteria, sampling frames, years of administration, or administrative procedures could all be involved" (p. 1057) in the difference. As will also be explored later, some researchers (Allen, 1994; Helzer, Spitznagel, \& McEvoy, 1987; Kulka et al., 1991; Newman et al., 1996) believe that even the DIS for the DSM III-R possesses low 
Table 1

General Population Estimates of the Prevalence of PTSD.

\begin{tabular}{llll}
\hline Study & Estimate & Instrument utilized & Sample size \\
\hline Helzer et al. (1987) & $0.5 \%$ (males) & DIS/DSM-III & 2,493 adults \\
& $1.3 \%$ (females) & & \\
& $1.0 \%$ (general) & & 2,985 adults \\
Davidson et al. (1991) & $1.3 \%$ (general) & DIS/DSM-III & 1,007 adults \\
Breslau et al. (1991) & $6.0 \%$ (males) & DIS/DSM-III-R & \\
& $11.3 \%$ (females) & & \\
Kessler et al. (1995) & $9.2 \%$ (general) & & 5,877 \\
& $5.0 \%$ (males) & DIS/DSM-III-R & \\
& $10.4 \%$ (females) & & \\
& $7.8 \%$ (general) & & \\
\hline
\end{tabular}

Note. The participants in Kessler et al. ranged in age from 15 to 54. 
Table 2

PTSD Prevalence Ranges for Males, Females, and General Population

Category

Range

Males

$0.5-6.0 \%$

Females

$1.3-11.3 \%$

General population

$1.0-9.2 \%$ 
diagnostic sensitivity and produces results that consistently underestimate the prevalence of all disorders, including PTSD. Despite concerns over changes in diagnostic criteria and instrument sensitivity, Green (1994) has stated it is clear that PTSD "is much more common in the general population than many other disorders" (p. 346).

Disorders Reported as Comorbid with PTSD

McFarlane and de Girolamo (1996) state that most trauma survivors develop a variety of comorbid disorders and from this they draw the conclusion that trauma and PTSD are not uniquely related. However, looking at the same facts, van der Kolk (1995) concludes that comorbidity has an adaptive function and is an attempt to manage PTSD symptoms. At this point, while there is some disagreement concerning the reasons why comorbidity exists, its abundant existence is beyond dispute. In fact, a letter by Kolb (1989a) in the American Journal of Psychiatry, states that "PTSD is to psychiatry as syphilis was to medicine. At one time or another PTSD may appear to mimic every personality disorder" (p. 811).

The earliest epidemiological studies that included PTSD consistently mentioned the fact that the disorder did not usually exist in isolation. Davidson and Fairbank (1993) noted a number of disorders that were identified as being comorbid with PTSD in both the North Carolina ECA and the St. Louis ECA. According to these authors, the former study included somatization disorder, schizophrenia, panic disorder, social phobia, obsessive-compulsive disorder (OCD), generalized anxiety disorder (GAD), depression, and drug abuse, among others as comorbid. The latter study identified $O C D$, dysthymia, manic depression, panic disorder, antisocial personality disorder (APD), phobias, drug abuse, and alcohol abuse as comorbid. Kolb (1989b) linked suicide, divorce, alcoholism, and unemployment to PTSD that has existed for more than three months and has thus become chronic.

Kessler et al. (1995) write that the studies by Helzer, Robins, and McEvoy (1987) and Breslau et al. (1991) identified comorbidity rates of $80 \%$ for those subjects with PTSD, while persons with psychiatric disorders other than PTSD had comorbidity rates of $30 \%$ and $43 \%$, respectively. Kessler et al. also found that individuals who have PTSD are much more likely to have a comorbid psychiatric disorder than individuals who do not 
Table 3

Odds Ratios for Disorders Comorbid with PTSD

Disorder

Odds ratio

Affective disorders

Major depression

Dysthymia

Mania

10.41

Anxiety disorders

Generalized anxiety

Panic disorder

Simple phobia

Social phobia

Agoraphobia

Substance disorders

Alcohol abuse/dependence

Drug abuse/dependence

Other

Conduct disorder

\subsection{5}

Note. Table adapted from Kessler et al. (1995). 
have PTSD. Table 3 is adapted from Kessler et al. (p. 1056) and presents these disorders and their accompanying odds ratios. These odds ratios indicate how much more likely it is for a male who has PTSD to have a specific co-occurring disorder than for a male who does not have PTSD.

It is also important for our later discussion to note Keane and Wolfe's (1990) finding that $26 \%$ of the PTSD-positive combat veterans they studied also had antisocial personality disorder (APD). This was close to research results from Kulka et al. (1990) who noted that $31 \%$ of combat veterans with PTSD evidenced comorbid APD. The study by Keane and Wolfe identified major depression and substance abuse as the diagnoses cooccurring most commonly with PTSD, with $84 \%$ also exhibiting major depression and $78 \%$ being identified as substance abusers. This finding was, once again, reinforced by Kulka et al. who found that $73 \%$ of their subjects with lifetime PTSD could be diagnosed as having a substance abuse problem and $26 \%$ could receive a diagnosis of major depression.

\section{Summary}

PTSD occurs relatively frequently within the general population and is associated with high rates of comorbidity with other psychiatric disorders. In fact, the prevalence rate for PTSD (5-6\% for males) exceeds or is comparable to many of the major disorders listed in DSM-IV (American Psychiatric Association, 1994), including major depression (5-12\% for males), dysthymic disorder (6\%), panic disorder (1.5-3.5\%), obsessivecompulsive disorder (2.5\%), and generalized anxiety disorder (5\%). However, the lifetime prevalence rate for the general population is often relatively small when compared to the rates for populations at risk for exposure to PTSD stressors, and it is to these rates that we will now turn our attention.

\section{Exposure Data}

\section{Vulnerability}

Yehuda and McFarlane (1995) note that it has not always been thought acceptable to identify factors that may make a person potentially vulnerable to the acquisition of PTSD symptoms. This was primarily because some felt this placed responsibility for the disorder with the individual who had it rather than on what or who precipitated it--a kind 
of "blaming the victim." However, PTSD research has progressed to the point where individual vulnerability can no longer be ignored. Briere (1997) states that the following variables have been identified as being associated with a greater likelihood of PTSD: gender, age, race, socioeconomic status, a previous psychological problem, inadequate coping styles, family dysfunction, and genetics. And Breslau, Davis, and Andreski (1995) would add high neuroticism and extroversion to the list. Individuals who have been exposed to a previous trauma or traumas may experience even stronger reactions to current stressors, something Post (1992) refers to as kindling. Briere refers to childhood trauma as a particularly important risk factor for revictimization. For example, Zaidi and Foy (1994) suggest that a person with a childhood abuse history is more likely to develop PTSD in response to combat exposure than those who were not the victims of abuse. But it may be that what is perceived as a risk factor is merely an increased likelihood of exposure to potentially traumatic events. In other words, females and Hispanics may be no more likely than males or whites to develop PTSD, just more likely to be exposed to the events that precipitate it. In addition, some researchers (Yehuda \& McFarlane) believe that trauma is an additive phenomenon. Although a traumatic response may not appear after the first trauma is experienced, it may develop after the second or third, especially if the events are in relatively close temporal proximity to one another.

But the variables cited by Briere (1997) and others do not appear to be consistent or inevitable predictors of the disorder. They vary from sample to sample and can depend on such things as the kind of trauma and population being examined, the instruments used to assess PTSD, and the size of the sample. Therefore, while the variables found by other researchers to be predictive of PTSD are valuable, their utility is limited, and they must be closely scrutinized for each new population.

\section{The Nature of Traumatic Stressors}

General information. Norris (1992) proposed the following definition of traumatic events: "The population of events involving violent encounters with nature, technology, or humankind" (p. 409), and McFarlane and de Girolamo (1996) wrote that "when the prevalence of such [precipitating] events is systematically examined, it is apparent that trauma is surprisingly commonplace" (p. 134). A wide variety of events, 
occupations, and activities can place an individual at risk for developing PTSD. Such stressful events, occupations, and activities include motor vehicle accidents (Blanchard et al., 1994; Burstein, 1989), violence at the hands of others (Caldwell, 1992; Davis \& Breslau, 1994; Nader, Pynoos, Fairbanks, \& Frederick, 1990); mental illness (Saxe et al., 1993); physical illness (Kutz, Gard, \& David, 1988); substance abuse (Cottler, Compton, Mager, Spitznagel, \& Janca, 1992; Dunn, Paolo, Ryan, \& Van Fleet, 1993); homelessness (North \& Smith, 1992); law enforcement (Folette, Polusny, \& Milbeck, 1994; Martin, McKean, \& Veltkamp, 1986; McCafferty, McCafferty, \& McCafferty, 1992); rescue work (Andersen, Christensen, \& Petersen, 1991; Fullerton, McCarroll, Ursano, \& Wright, 1992); natural disasters (Green \& Lindy, 1994; Green et al., 1990; Madakasira \& O'Brien, 1987; McFarlane, 1988; McFarlane \& Papay, 1992; Steinglas \& Gerrity, 1990); prostitution and exotic dancing (Ross, Anderson, Heber, \& Norton, 1990); serving in the military under combat conditions (Friedman, Schnurr, \& McDonagh-Coyle, 1994; Hyer, Summers, Braswell, \& Boyd, 1995; Kulka et al., 1990; Solomon et al., 1992; True et al., 1993; Weiss, Marmar, Schlenger, \& Fairbank, 1992), and sexual and physical abuse (Breslau et al., 1991; Foa, Rothbaum, \& Steketee, 1993; Harvey \& Herman, 1992; Kilpatrick, Saunders, Veronen, Best, \& Von, 1987). Due to the fact that the literature in this area is so extensive and somewhat redundant, information will be provided from only a sample of PTSD prevalence studies.

Motor vehicle accidents. The most important study on PTSD and motor vehicle accidents was conducted by Blanchard et al. (1994) who looked at 50 accident victims. Forty-six percent of these individuals were given a diagnosis of PTSD. Almost half of the PTSD-positive group also met the criteria for major depression. These researchers used the Clinician-Administered PTSD Scale (CAPS) to diagnose PTSD, which Scotti (in press) has referred to as the "gold standard" when it comes to PTSD assessment. Norris (1992) found that while motor vehicle accidents were less frequent and severe than some other stressors (e.g., tragic death and sexual assault, respectively), the combination of frequency and severity made this type of event perhaps the most important of all she studied. 
Injuries and assaults. McFarlane and de Girolamo (1996) report that 21 studies have looked at violence and PTSD. Only three of these studies identified prevalence rates that were less that 25\%. For example, Shalev, Peri, Canetti, and Schreiber (1996) studied 53 subjects who had received injuries as a result of traumatic events and found that $25.5 \%$ met the criteria for PTSD six months after the event. However, it seems as though rape is one of the most serious traumatic stressors. Breslau et al. (1991) reported that of the 16 women in their survey who reported having been raped, $80 \%$ were diagnosed with PTSD. Kilpatrick et al. (1987) assessed 391 adult females for their reactions to criminal victimization. Of the 391 participants, $23.3 \%$ had experienced a completed rape and $57.1 \%$ of these women developed PTSD. Foa et al. (1993) believe that victims of rape may comprise the largest group of persons experiencing PTSD. Kilpatrick et al. also noted that $36.8 \%$ of women who experienced an aggravated assault developed PTSD.

Occupations and PTSD. Some occupations can also expose individuals to potential trauma more than others and we would expect to see higher rates of PTSD and associated disorders among these individuals. Fullerton et al. (1992) cited the National Commission on Fire Prevention and Control, which said that "fire fighting is the most hazardous and dangerous occupation in the United States" (p. 371). This evaluation was confirmed by McFarlane (1988) who studied 469 volunteer Australian firefighters who had worked to extinguish a wild fire and found a current PTSD rate of $16 \%$ among this group. Martin et al. (1986) looked at police officers who had worked with crime victims or had been victimized themselves and discovered a current PTSD prevalence of $26 \%$, which is the same as the rate of PTSD cited in McCafferty et al. (1992) for active duty San Diego police officers in 1984.

Homelessness. North and Smith (1992) found the lifetime prevalence of PTSD among 600 homeless men and 300 homeless women in St. Louis to be $18 \%$ and $34 \%$, respectively. Rape was the stressor most likely to produce PTSD in both men and women. Also of interest was the researchers' discovery that three quarters of these individuals had PTSD before they began living on the streets. Therefore, even though PTSD rates are high for this group, it is unclear whether the disorder precipitates or maintains homelessness or both. 
Substance abuse and PTSD. Cottler et al. (1992) examined a substance abusing sample of the 1984 St. Louis ECA and discovered that cocaine/opiate users were nearly four times more likely to meet the criteria for PTSD than were members of a non-using comparison group. Dunn et al. (1993) also noted that $16 \%$ of a substance abuse population had Dissociative Experiences Scale (DES) scores over 30 and that such scores are usually associated with PTSD or Dissociative Identity Disorder (DID). These researchers felt that substance abuse often masks dissociation and leads to underestimates of the problem in this population. They also noted that African-Americans had significantly higher DES scores than whites.

PTSD and physical illness. Kutz et al. (1988) and Kutz, Shabtai, Solomon, Neumann, and David (as cited in Green et al., 1997) looked at the incidence of PTSD among people who had experienced myocardial infarctions (MI). In both studies, the authors found that $15 \%$ of post-MI patients had chronic PTSD (present three months or longer), while an additional 5-10\% of this population sample had suffered from the acute form of PTSD (present less than three months). Green et al. (1997) also cite a number of studies indicating that a cancer diagnosis may precipitate PTSD symptoms.

PTSD among veterans. A number of studies have looked at the prevalence of PTSD among veterans and have come to conflicting conclusions. Earlier studies using methodology similar to that used in the ECA studies tended to show lower rates of lifetime prevalence for the disorder in this population. However, studies that used multiple measures of PTSD to confirm the diagnosis tended to show rates that were much higher than the rates for the general population (Kulka et al., 1991). Perhaps the most comprehensive and methodologically sophisticated study of veterans was the National Vietnam Veterans Readjustment Study (NVVRS) (Kulka et al., 1990). This study identified a lifetime prevalence rate for PTSD of $30.9 \%$ in males and $26.9 \%$ in females who had been in the Vietnam theater of operations. Friedman et al. (1994) looked at male Persian Gulf veterans who evidenced only a 3.2\% rate of PTSD upon returning home, but a $9.4 \%$ prevalence rate 18 months later.

McFarlane and de Girolamo (1996) looked at 11 studies that assessed PTSD in former prisoners of war and political prisoners and reported that six of those studies 
identified prevalence rates of $50 \%$ or higher, while three studies identified rates of $70 \%$ or more. They also stated that research into victims of terrorist attacks found rates of 20$40 \%$ and higher.

PTSD and natural disasters. The results in this area seem mixed. Shore, Vollmer, and Tatum (1989) conducted a study involving victims of the Mt. St. Helen's volcanic eruption, which showed a lifetime prevalence for PTSD of $3.6 \%$ compared with $2.6 \%$ in controls. Steinglas and Gerrity (1990) looked at the effects of having to leave one's home in two communities following a natural disaster. In one community, current PTSD was $14.5 \%$ at four months post-disaster and $4.5 \%$ at 16 months post-disaster. In the other, it was $21 \%$ at 16 months post-disaster. The authors speculated that community composition and unity may have had something to do with the course of the disorder. Green et al. (1990) re-examined survivors of a 1974 Buffalo Creek, WV, dam burst using the Structured Clinical Interview for the DSM-III (SCID) and found a PTSD prevalence rate of $28 \%$, which was down from $44 \%$ immediately following the disaster. The researchers stated that those who were still PTSD-positive so long (12 years) after the event "had more extreme and prolonged life-threatening experiences and loss of closer family members" (p. 51) than those who had recovered by then. Madakasira and O'Brien (1987) also studied the impact of tornadoes in North and South Carolina and found that $59 \%$ of their sample of 116 disaster victims qualified for a PTSD diagnosis. They also stated that this is within the range of PTSD for man-made disasters (45-81\%) reported by other researchers.

Conclusions. This review of populations that have been exposed to a variety of PTSD stressors indicates that the prevalence of the disorder is usually much higher than in the general population. However, it is often difficult to compare studies due to methodological inconsistency. In general, those studies that used the Diagnostic Interview Schedule as their main or only instrument report prevalence rates that are lower than those studies that used the Structured Clinical Interview for the DSM (SCID), the Clinician- Administered PTSD Scale (CAPS), or multiple measurements.

Another problem with all PTSD studies is that genetic predisposition may be a relatively unrecognized confounding factor. True et al. (1993) found that genetic 
inheritance has a substantial impact on an individual's liability for various PTSD symptoms. In their study of twins, genetic factors accounted for $13-30 \%$ of the variance for DSM-IV (1994) re-experiencing symptoms; $30-34 \%$ of the variance for avoidance; and $28-32 \%$ for arousal. In addition, there was no evidence that shared environment contributed to the development of PTSD. However, this research also indicates that stressors still account for approximately three quarters of the variance for the development of PTSD.

We will now turn our attention to studies of the incidence of PTSD among prison inmates in an attempt to determine how prevalence has been assessed in this population and whether inmates seem to be more like the general population or an at risk population.

Mental Disorder and Prison Inmates

\section{Mental Disorder and Criminality}

The general relation. Before looking at the prevalence among inmates of mental disorders in general and PTSD in particular, it would be helpful to know if any link has been established between mental disorders and criminality. Hodgins (1993) states that mental disorders vary in their course, symptomatology, etiology, and response to treatment, and that it is reasonable to assume that they will also be differentially associated with crime. In fact, she suggests that some disorders may actually inhibit criminality while others exacerbate it.

To assess the general relation between mental disorder and criminality, Link, Andrews, and Cullen (1992) looked at the criminal behavior of psychiatric patients and subjects who had never received any mental health treatment. The subjects, all of whom were from the same New York neighborhood, were divided into four cohorts: a community sample and three groups of subjects with increasing degrees of contact with the mental health system. Only $6.7 \%$ of the community sample had ever been arrested. A comparable number of those individuals with the least contact with the mental health system $(6 \%)$ had been arrested, while approximately $12 \%$ of those with more substantial contact with the mental health system had been arrested. More of the patients were arrested for felonies and violent behavior than were the subjects in the community sample. Hodgins (1993) also states it has been demonstrated that patients discharged 
from psychiatric wards are more likely to commit crimes than the nondisordered individuals from their communities, and that this is especially the case for males.

Longitudinal and birth cohort studies. Lindqvist and Allebeck (1990) followed the behavior of 644 Swedish males with schizophrenia for 14 years to determine whether or not their criminal behavior differed from that of the general public. What the researchers discovered was that although the persons with schizophrenia were no more likely to commit crimes than the general public, they committed four times more violent offenses than persons without schizophrenia. Ortmann (as cited in Hodgins, 1993) examined the criminal and psychiatric records of 11,540 men born in Denmark in 1953, and discovered that $34.8 \%$ of all males without a disorder had at least one conviction, $43.5 \%$ of males with a major psychiatric disorder had been convicted, $83.2 \%$ of those with drug/alcohol abuse/dependence had been convicted, and $50.9 \%$ of those with other diagnoses had been convicted. Concerning a Swedish cohort of 15,117 persons residing in Stockholm in 1963, Hodgins (1993) notes that males with a major mental disorder (defined as those manifesting psychoses, schizophrenia, affective psychoses, and paranoid states) had a conviction rate of $47.6 \%$, while those with a substance abuse or dependence problem had a conviction rate of $\mathbf{8 9 . 7 \%}$. However, there was no difference in conviction rates between males without any mental disorder and those with a less-than-major disorder $(29.4 \%$ and $34.4 \%$, respectively). No information was provided concerning individuals with a dual diagnosis.

Based on these studies, it seems that individuals with a major mental disorder or with alcohol or drug problems are at increased risk for criminal and violent behavior. The Prevalence of Mental Disorders Among Inmates

Teplin (1990b) and Steadman, Fabisiak, Dvoskin, and Holohean (1987) noted the paucity of accurate studies concerning the prevalence of mental disorders among prisoners. Teplin provided a summary of 18 research studies conducted during the period 1976-1986 concerning the prevalence of mental disorders. She found that the studies showed little consistency and cited a number of factors that may have been responsible for this including a lack of randomized sampling, unreliability of measurement instruments, unspecified diagnostic criteria, and sample sizes that were too small. Teplin 
sought to correct this state of affairs by comparing the current and lifetime prevalence of schizophrenia, major depression, and mania among 728 Cook County, IL, jail detainees with that of the population in general as measured by the ECA studies combined for five cities. To make her results comparable with those of the ECA, she used the DIS for DSMIII. Teplin found that the prevalence rates for schizophrenia, major depression, and mania among jail detainees were two to three times higher than among the general population, and that "the observed ratio of current jail rates to current population rates is substantially higher than the comparable ratio of lifetime rates" (p. 666). This led her to conclude that, for the sample studied, arrests were made during an active phase of their mental disorder. The results of this study add credence to an already established link between mental illness and crime.

Daniel et al. (1988) took Teplin's work one step further by conducting a study comparing lifetime and six-month prevalence rates for female offenders with the general population of the St. Louis ECA for a wider range of disorders, including substance abuse, anxiety disorders, anorexia nervosa, and antisocial personality disorder. By not limiting their study to schizophrenia, major depression, and mania, these researchers found that the most prevalent mental disorders among female offenders were alcohol abuse and dependence (36\%), drug abuse (26\%), APD (29\%), and depressive disorders (22\%). All of these were significantly higher than prevalence rates in the general population.

Hodgins and Cote (1990) provided a key summary of American state prison research and compared it with research of their own conducted at a penitentiary in Quebec. Two studies, one by Collins and Schlenger in Ohio and one by Neighbors et al. in Michigan (as cited in Hodgins and Cote) used the DIS for the DSM-III (1980) to arrive at prevalence estimates of mental disorders among inmates. The results of these studies were subsequently confirmed by another study conducted by Hyde and Seiter (as cited in Hodgins and Cote) using the SCID for DSM-III-R. Unfortunately, Hodgins and Cote only provide prevalence ranges for these American studies and do not tell us which studies arrived at what results. Furthermore, attempts to obtain copies of the studies were 
Table 4

Prevalence Rates of Mental Disorders for U. S. and Canadian Inmates

Disorder

U. S.

Canada*

General population**

Major disorders

Bipolar disorder

$1.1-3.8$

4.8

$0.7-1.1$

Major depression

$5.3-12.7$

16.9

$2.1-5.9$

Organic brain disorder

$0.4-5.3$

0.4

$0.9-3.1$

Schizophrenia

$1.2-2.8$

7.5

$0.5-1.2$

Substance abuse/dependence

Alcohol abuse

$4.5 \quad 15.2$

Alcohol dependence

24.1

5.5

Combination

$* * *$

46.2

Drug abuse

6.9

17.0

Drug dependence

Combination

21.8

4.9

*** $\quad 27.5$

$* * *$

***

$* * *$

$* * *$

$* * *$

$* * *$

Less serious disorders
APD
$28.9-50.1$
61.7
$2.1-6.5$
Bulimia
***
0.6
Dysthymia
$2.0-6.4$
13.8
$1.2-2.6$
GAD
$1.3-22.0$
61.2
OCD
$0.6-5.6$
7.5
$1.1-2.8$
Panic disorder
$0.9-1.6$
3.2
$0.6-1.2$
Phobias
$3.8-45.8 \quad 34.4$
$5.3-19.7$
PTSD
$2.3-6.0$
13.0
$0.5-6.0$
Somatization
0.3
1.0
***

(table continues) 
Table 4 (continued)

* Figures derived from a correctional facility in Quebec.

** Figures derived from ECA studies and a Canadian sample.

*** Not estimated.

Note. All figures, with the exception of the "PTSD General Population," Hodgins and Cote (1990). "PTSD General Population" Table 2, this study. 
unsuccessful. Therefore, it impossible for us to tell how prevalence rates obtained using the DIS and the SCID compared. However, the combined studies do indicate that inmates are much more likely to experience a mental disorder than the general male population (see Table 4). This holes true for major mental disorders, drug and alcohol abuse and dependence, and what are referred to as "less serious mental disorders" (Hodgins \& Cote, p. 3).

\section{Co-occurring Mental Disorders Among Inmates}

Cote and Hodgins (1990) noted that mental disorders seldom appear in isolation among inmates. For this population, comorbidity appears to be the rule, rather than the exception. They cite an unpublished 1988 study by Mirsky, indicating that " $78 \%$ of schizophrenics, $82 \%$ of those who met the criteria for bipolar disorder and $78 \%$ of those with a major depression received three or more additional diagnoses" (pp. 271-272). Cote and Hodgin's own study of comorbidity tended to confirm these earlier findings. In their study, 55.3\% of inmates with schizophrenia, $64.7 \%$ of those with bipolar disorder, and 43.8\% of inmates with major depression received three or more additional diagnoses, while another $36.8 \%$ of those with schizophrenia, $29.4 \%$ of those who were bipolar, and $50.6 \%$ of those with major depression received one or two additional diagnoses.

Teplin (1984) noted that individuals with multiple diagnoses may be more likely to land in prisons rather than treatment centers for the simple reason that they present an ambiguous clinical picture. Cote and Hodgins (1990) point out that there may be a link between violent behavior and comorbidity. For example, they note that individuals with dual diagnoses including antisocial personality disorder (APD) are more likely to use weapons when intoxicated than people who are just alcohol dependent. In addition, Cote and Hodgins state that comorbidity can affect symptom severity, the course of a disorder, the client's response to treatment, and the clinician's response to the client. In other words, comorbidity seems not only common in this population, but problematic, as well. As we saw earlier, comorbidity, including such likely comorbid disorders as major depression, generalized anxiety disorder (GAD), substance abuse and dependence (Kessler et al., 1995), and APD (Keane \& Wolfe, 1992, Kulka et al., 1990), is one of the hallmarks of PTSD. 


\section{The Prevalence of PTSD Among Inmates}

Collins and Bailey (1990) used the DIS for the DSM-III (1980) to estimate a lifetime prevalence of PTSD among a sample of 1140 incarcerated male felons in North Carolina. Of this sample, 29 inmates were diagnosed as having had PTSD, for a lifetime prevalence rate of 2.3\%. As the prevalence of PTSD identified by Davidson et al. (1991) for the North Carolina ECA was 1.3\%, the figure of $2.3 \%$ represents an increase over the rate for the general population.

Hodgins and Cote (1990) note that PTSD rates established for other American state prisons using the DIS for DSM-III (1980) and the SCD for DSM-III-R (1987) were $6 \%$, while the rate of PTSD was $13 \%$ in a Canadian penitentiary using a French version of the DIS for DSM-III. Based on what we have seen thus far (i.e., consistent increases in PTSD estimates from DSM-III to DSM-III-R and improved results using the SCID), these figures and those from Collins and Bailey (1990) seem contradictory. Hodgins and Cote state that generalizing the results of American studies to Quebec, Canada, is somewhat problematic due to a difference in penitentiary populations. The authors also note that their results, even though higher than those for American state penitentiaries, may be lower than what actually exists. One major difference in the Canadian study is the fact that inmates were guaranteed confidentiality of results in all but the most extreme cases, and this may have allowed inmates to tell the truth about their disorders.

The DIS has been shown to underestimate mental disorders. According to Hodgins (1995), one of the reasons for this is that the DIS was not designed to detect major mental disorders in people experiencing multiple disorders. A lifetime PTSD prevalence rate of $2.3-6 \%$ among American male inmates also seems very low, given the fact that epidemiological estimates cited earlier estimate the lifetime prevalence for males in the general population as between 5-6\%. If these figures were correct, then it would mean that there is no difference in the lifetime prevalence rate for male inmates and males in the general population. However, study after study has already confirmed that rates of other mental disorders are generally higher in the prison population than the general population. Therefore, it stands to reason that the Quebec prevalence rates are closer to 
the actual rates than the American rates, even though they may still be underestimates of the actual prevalence rate for PTSD.

In a more recent study, Teplin, Abram, and McClelland (1996) looked at 1,272 female jail detainees in Chicago, IL. Using the DIS for the DSM-III-R (1987), these researchers identified a lifetime PTSD prevalence of $33.5 \%$ among this population and a six-month prevalence rate of $\mathbf{2 2 . 3 \%}$. Given that PTSD prevalence rates for women are usually twice those of rates for men, these results might also be an indication that Hodgins and Cote's (1990) results are closer to actual levels than prevalence rates attained in other studies.

But even if this is the case, why should we care? Does it matter if PTSD prevalence rates for inmates are underestimated? These are the questions to which we will now turn our attention.

\section{Mental Disorder, Prison Inmates, and Society}

\section{Why Provide Mental Health Services to Prisoners?}

Ogloff et al. (1993) provide four reasons why inmate mental health needs to be assessed:

1) to identify inmates who may be at risk for injurious behavior to themselves or others; 2) to determine whether an inmate is so mentally ill that he or she cannot participate in prison activities; 3) to determine whether an inmate is so mentally ill that he or she should be transferred to a mental health facility; and 4) to determine whether an inmate who is not so mentally ill as to be transferred to a mental health facility can benefit from treatment (p. 63).

Danger to self and others. In the first instance, mental health personnel are primarily attempting to determine whether or not the inmate is at risk for suicide or is likely be become aggressive toward another inmate or staff member. Reinforcing this as the number one priority, Dvoskin and Steadman (1989) state that one of the miajor reasons inmates are provided with mental health services is to "help make the prison a safer place for both inmates and staff' (p. 205). Researchers van der Kolk and McFarlane (1996) state that "the personal meaning of the traumatic experience evolves over time, and often 
includes feelings of irretrievable loss, anger, betrayal, and helplessness" (p. 9). Although these feelings may lie dormant for long periods of time, a new stressor, such as incarceration or violence within the prison, may bring them back to life. In addition, van der Kolk and McFarlane note that traumatized individuals may feel themselves compulsively drawn to situations that are similar to the original trauma. They indicate that having been physically or sexually abused as a child is a common thread through the past of many violent offenders, and that such childhood experiences often result in the subsequent abuse of others. van der Kolk and MacFarlane also state that:

people with PTSD tend to move immediately from stimulus to response without actually realizing what makes them so upset. They tend to experience intense negative emotions (fear, anxiety, anger, and panic) in response to even minor stimuli; as a result, they either overreact and threaten others, or shut down and freeze...Perhaps the most distressing aspect of this hyperarousal is the generalization of threat. The world increasingly becomes an unsafe place (p. 13).

In addition, van der Kolk and McFarlane note a link between childhood abuse and selfdestructive behavior, including suicide attempts. As has been stated previously, individuals with PTSD are more likely to have comorbid major depression than those without PTSD (Kessler et al., 1995), and major depression is often a precursor of suicide.

Hodge (1992) believes that early traumatic experience of sexual and physical abuse, as well as adult experiences of such things as combat, can lead an individual to become "addicted" to violence. He views antisocial personality disorder as a syndrome acquired via trauma and fed by addiction. For Hodge, addiction is not to a substance but to an experience. According to his theory, some trauma survivors, in attempting to recreate an "adrenaline high" associated with their original trauma, become addicted to the sensation they seek. He writes that "the greater the exposure to the substance or behaviour, the stronger the compulsion" (p. 220).

However, the most compelling evidence that inmates with PTSD may be a danger to others comes from Collins and Bailey (1990), who studied 1,140 incarcerated male 
felons. These authors found that PTSD is linked to what they called "serious expressive violence," which they operationalized to include homicide, rape, and violent assault. Collins and Bailey noted that in their sample, PTSD usually preceded violent behavior. Therefore, it seems clear that identifying inmates with PTSD will make the institution safer for the inmate himself, for other inmates, and for the corrections staff.

Benefit from prison activities. The second objective has to do with determining whether or not an individual can participate and benefit from prison activities. Two important Federal prison programs serve as examples. The first involves allowing the inmate to obtain his high school equivalency certificate (GED). The second is a biopsychosocial Residential Drug and Alcohol Program (R-DAP) designed to help drug and alcohol abusers gain a sense of mastery over their problems. The existence of PTSD could make it more difficult for an inmate to complete either program.

In the case of the GED program, van der Kolk and McFarlane (1996) note that people with PTSD have trouble with attention, working memory, distractibility, and visualization, all of which will contribute to one's success or failure in educational programs. van der Kolk (1996b) also cites studies that show high rates of alcohol and drug abuse among traumatized adults. He observes that "it is likely that substance abuse treatment of traumatized individuals can be more effective if the issue of recurrent posttraumatic problems during withdrawal is vigorously addressed" (p. 191). The combination of PTSD and drug and alcohol problems may make it more difficult for inmates to succeed in programs such as R-DAP. Therefore, it also seems as though the identification of individuals with PTSD is important if officials want them benefit from prison education and rehabilitation programs.

Need to be transferred. The third factor involves evaluating whether or not an individual could be better served in an institution designed to treat inmates with mental disorders. Given that posttraumatic stress disorder is associated with violent behavior (Collins \& Bailey, 1990), suicidal ideation (Briere, 1997), a high rate of comorbidity with other disorders (Kessler et al., 1995), and can be active for many years (Green, 1994; Kulka et al., 1990), the transfer of some inmates may be a reasonable option. The potential level of pathology associated with PTSD also indicates that it is not only 
necessary to screen inmates for the disorder, but to follow-up those screens with in-depth interviews and assessments.

Capacity for treatment. Finally, we have the question of treatment. Researchers van der Kolk, McFarlane, and van der Hart (1996) state that "although posttraumatic stress disorder (PTSD) is among the most common psychiatric disorders...systematic investigation of what constitutes effective treatment is still in its infancy" (p. 417). Meichenbaum (1994) agrees. Researchers van der Kolk, McFarlane, and van der Hart (1996) report that some programs may even have negative outcomes for patients and cite an Israeli-developed treatment approach (the Koach project) that actually increased symptoms and disabilities in a number of areas. Furthermore, Meichenbaum states that the evidence is simply not yet available to suggest the clear superiority of one form of treatment over another. Therefore, we must exercise care when treating individuals with PTSD. Despite this lack of certainty, van der Kolk et al. state that "psychotherapy must address two fundamental aspects of PTSD: 1) deconditioning of anxiety, and 2) altering the way victims view themselves and their world by reestablishing a feeling of personal integrity and control" (p. 420). Admittedly, both of these are extremely difficult to achieve in a prison. In fact, one wonders if the prison environment does not add to feelings of anxiety, as well as diminish an individual's personal integrity and control. In this sense, it is even possible that incarceration is itself a traumatic stressor.

Conclusion. Having reviewed four reasons for assessing mental disorder in prisoners, with particular regard for PTSD, it has become clear that it is important to know whether or not an inmate has the disorder. However, if this diagnosis is already being made by prison authorities, then the issue is moot. Therefore, it is necessary to turn our attention to how mental health disorders are identified in America's jails.

\section{Identification of Mental Disorders}

\section{Among Individuals}

As previously mentioned, Teplin (1990a) examined a random sample of 728 Cook County, IL, inmates using the DIS for the DSM-III to determine the factors that influenced treatment decisions. She found that there were a number of variables that helped determine whether or not a mental disorder would be detected. The first and most 
influential of these was a notation of treatment history in jail records. The second was behavioral problems observed and noted in jail records. The third was the nature of the individual's arrest. The fourth was obvious depressive symptoms which had been entered into the formal record. The fifth was exhibiting schizophrenia. Teplin writes that "the effect of psychiatric history on detection was so strong that its importance superseded the true presence or absence of schizophrenia" (p. 235). Although jail personnel had identified only $32.5 \%$ of all severely ill detainees, including $45 \%$ of those with schizophrenia and $7 \%$ of those with major depression, $91.7 \%$ of those with an identified treatment history were detected as having a mental illness. Because the degree of detection of mental disorder is bound to vary from institution to institution, it is possible that Teplin has identified a kind of default state--one that will take place if nothing is done to alter the situation intentionally. This means that to be able to identify inmates with PTSD, corrections officials will have to look for them.

\section{Among Groups}

To identify inmates with PTSD, it would be helpful if one knew whether or not this disorder is more prevalent among different types of prison populations. For example, as females have been shown to have higher rates of PTSD than males (Breslau et al., 1991; Helzer et al., 1991), it seems likely that female inmates will also evidence higher rates of PTSD than male inmates. Findings by Teplin et al. (1996) appear to confirm this.

McFarlane and de Girolamo (1996) have also noted that PTSD stressors can be acute (i.e., a natural disaster), cumulative (i.e., the repeated events to which police are exposed), or extended (i.e., long-term sexual abuse). In the case of cumulative stressors, this means that the environment in which a person lives his or her life or the individual's previous experiences may increase the likelihood of a PTSD diagnosis. Breslau, Davis, and Andreski (1995) have shown that traumatic stressors are not necessarily random events, and that individuals who have been exposed to a traumatic stressor are twice as likely to be exposed to another traumatic stressor than individuals without prior exposure. Resnick, Yehuda, Pittman, and Foy (1995) noted that rape victims with a prior history of sexual abuse were more likely to develop PTSD than rape victims without such a history. 
If it is the case that stressors are cumulative and, in a sense, self-perpetuating, then this may help us identify segments of the prison population at increased risk for PTSD. For example, prison security levels are a function of violence, severity of the crime, and the number of past offenses committed by an inmate (US Department of Justice, 1995a). This indicates that there may be a relation between an inmate's security level, the quantity and severity of stressors he has experienced, and the development of PTSD. Therefore, we might hypothesize that there is a positive correlation between security level and PTSD.

McNally and Shin (1995) have also shown that full-scale IQ is a significant predictor of PTSD. These researchers studied 105 male Vietnam combat veterans, and determined that severe PTSD symptomatology could not only be predicted by low IQ, but that IQ was a better predictor of PTSD symptoms than combat exposure. The average level of education for state inmates is only the 11 th grade and more than $60 \%$ of all state inmates have not completed high school (U. S. Department of Justice, 1994b). If federal figures are of a similar magnitude, then it makes sense that one place to look for PTSD would be among the less educated portion of the inmate population.

Davidson et al. (1991) have noted that such factors as parental poverty, history of child abuse, and parental separation prior to age 10 are predictors of PTSD. McCranie, Hyer, Boudewyns, and Woods (1992) state that having experienced negative parenting, as defined by "rejection and punitive/hostile methods of discipline and control" (p. 433), can contribute to PTSD. In their study of homeless men and women, North and Smith (1992) identify childhood histories of abuse and family fighting as predictors of PTSD. Although such things as child abuse, negative parenting, family fighting, and parental separation are not the exclusive province of families of lower socioeconomic status (SES), it is not unreasonable to expect a higher rate of such occurrences among such families. In any event, parental poverty is a characteristic of such families.

Kulka et al. (1990) also found that the prevalence of PTSD among Vietnam theater veterans differed according to race. Although Kulka et al. identified an overall rate of current PTSD among veterans of $15.2 \%$, the current rate for Hispanics was $27.9 \%$, for African-Americans it was $20.6 \%$, and for Caucasians/others it was $13.7 \%$. However, 
Manson (1997) points out that this does not mean that Hispanics and African-Americans are more susceptible to PTSD than are Caucasians. In fact, he reports that when combat exposure was controlled, the differential rate between Caucasians and African-Americans disappeared and was sharply reduced for Hispanics. It may be that due to lower socioeconomic status or education, African-Americans and Hispanics were more likely to be in combat and, therefore, were more likely to experience trauma and develop PTSD.

Finally, Foa (1995) has noted that $14 \%$ of the sample of 248 individuals used to establish norms for her Posttraumatic Stress Disorder Scale (PDSTM) indicated that imprisonment had been a stressor in their lives. Although no one in this non-incarcerated sample identified imprisonment as a precipitant of PTSD, the results might be different and worth investigating for men who are still in prison. This researcher believes that events preceding incarceration, such as arrest, indictment, and sentencing, may also be traumatogenic.

\section{Summary}

Although some epidemiological studies indicate the lifetime prevalence of PTSD among American males to be $5-6 \%$, results vary according to the assessment instruments and diagnostic criteria employed. This is also the case when it comes to assessing populations that have been exposed to specific PTSD stressors such as assault, combat, natural disasters, motor vehicle accidents, life-threatening illness, homelessness, mental illness, dangerous occupations, substance abuse, and sexual and physical abuse. Despite a lack of analytical uniformity, it is clear that the prevalence rate of PTSD rises dramatically for at-risk groups.

PTSD seldom presents by itself and is usually accompanied by comorbid disorders such as major depression, GAD, substance dependence and/or abuse, and APD. In addition, researchers have found that comorbidity is more likely in the case of PTSD than with other psychiatric disorders and that, over time, some comorbid disorders become independent of PTSD. This indicates that the presence of PTSD symptoms can have a wide-ranging impact on the manner in which an individual lives his or her life and the way other individuals and institutions will respond to the person who is PTSDpositive. 
Studies in the United States and Europe have indicated that there is a relation between mental illness and crime, and that persons with mental disorders not only commit more crimes, but more violent crimes, as well. Therefore, the issue of the degree of mental illness among the inmate population is important. Just as is the case in the general population, comorbidity is an important factor in the prison setting. In fact, some researchers believe that patterns of comorbidity, as opposed to simple mental disturbance, may play an important role in the commitment of crimes by individuals with mental disorders.

Epidemiological studies of prison inmates in the United States appear to indicate that the rate of lifetime PTSD among this population is substantially higher than that of the general population. However, the results of these studies are confusing and vary widely. Some studies used the DIS for the DSM-III, while one used the SCID for the DSM-III-R. Based on experience, the results of epidemiological studies using different instruments would be expected to produce substantial differences in prevalence rates for inmates over baseline rates. But this has not always been the case. One study that employed the DIS for the DSM-III showed the kind of increase one would expect (6\% as opposed to $1 \%$ in the general population), while another did not ( $2.3 \%$ compared to $1.3 \%$ ). Furthermore, a survey using the SCID also showed a $6 \%$ prevalence rate. Another epidemiological study conducted in Canada also used the DIS for the DSM-III and arrived at a PTSD prevalence rate of $13 \%$. However, as it has been repeatedly shown that the DIS lacks diagnostic sensitivity, the actual rate of lifetime PTSD among prison inmates may well be higher than $13 \%$. What is clear, however, is that there is a need to use the most sensitive diagnostic instruments available to arrive at an accurate estimate of the prevalence of PTSD among male inmates. This leads to this study's first hypothesis: - that the current and lifetime rates of PTSD among male inmates will be higher than those exhibited in the general population and higher than those identified in previous estimates involving American inmates.

In addition, it has been found that PTSD stressors are not only cumulative in the sense that an individual is more likely to develop PTSD if $s /$ he has experienced a prior stressful event, but the existence of prior stressors also predispose one to experience 
additional stressful events, so that PTSD might be considered self-fulfilling. If this is the case, then one might expect inmates with a more violent past and multiple offenses to have a higher prevalence of PTSD than those who are less violent and have committed fewer offenses. Such a pattern is congruent with increasing prison security levels, and leads to the second hypothesis:

- that inmates incarcerated in a medium security facility will manifest a higher prevalence of PTSD than those in a minimum security facility.

However, it has also been found to be the case that unless mental illness is a part of an individual's formal record, then it is likely to go unnoticed by corrections officials, which leads to a third hypothesis:

- that the majority of inmates who are identified as PTSD-positive will not have been previously identified as such by corrections officials.

But even if it is determined that the incidence of PTSD among prison inmates is higher than the general population, that it increases with security level, and that corrections authorities are usually unaware of the existence of the problem, are these important findings? A variety of factors indicate that the answer to this question is an unequivocal "yes."

There are a number of reasons why we assess inmates for mental disorders. The first is to determine whether or not they are a danger to themselves and others. Although the literature indicates that there is a general link between mental disorder, crime, and violence, it also provides strong evidence that individuals who are PTSD-positive may have a tendency to be harmful of themselves and others.

The second reason assessment is necessary is to determine whether or not inmates can participate in prison programs that may improve their lives, such as educational and drug and alcohol programs. Once again, the literature indicates that the presence of PTSD may well inhibit one's ability to concentrate and to learn and may impinge upon the efficacy of prison rehabilitation programs. Due to the high rate of comorbidity between PTSD and drug and alcohol abuse, some researchers believe that treatment will be more effective if the posttraumatic stress is addressed as part of the recovery. Therefore, because residential drug and alcohol treatment is a major program in some federal 
minimum security facilities, it would be important to have an estimate of the prevalence of PTSD among the inmate population.

The third assessment objective is to determine whether or not an inmate should be transferred to an institution with a better capacity to treat mental illness. In some cases, the severity of PTSD symptoms may be such that this is the case. In others, it may be the comorbid disorders that will precipitate a transfer.

Finally, assessment helps determine whether or not an inmate can benefit from treatment. The treatment of PTSD in a prison setting can be problematic due to issues of on-going anxiety and trust. In fact, it may even be the case that incarceration itself is a PTSD stressor. If this is so, then it would certainly be important to be aware of this possibility. Therefore, a fourth hypothesis is:

- that, for some inmates, incarceration itself will have been a traumatic stressor.

Clearly, it would be helpful to have a sense of who might be vulnerable to PTSD, as this knowledge would allow corrections officials to target these populations for priority screening and permit a strategic allocation of limited resources. In this regard, it has already been noted that certain factors, including race, IQ, and SES predispose an individual to PTSD. To determine whether or not this is the case, the fifth, sixth, and seventh hypotheses will be tested:

- that PTSD-positive inmates will have lower full scale intelligence quotient (IQ) estimates than inmates without PTSD.

- that the childhood socioeconomic status of PTSD-positive inmates will be lower than that of inmates without PTSD.

- that African-American and Hispanic inmates will have higher PTSD prevalence rates than Caucasian inmates.

Now that a basic rationale for this undertaking has been established, what remains is a description of how to go about achieving the research objectives described above. 


\section{Chapter 3: METHOD \\ Introduction}

Roesch et al. (1995) note that divergent prevalence rates are often the result of differences in methodology and in the populations being examined. For inmate populations, this means that prevalence rates may well vary according to whether one is examining federal, state, or local prisoners or whether one is examining inmates in the United States or another country. Therefore, Roesch et al. believe that it may be necessary for each institution or type of institution to evaluate the prevalence of mental disorders within its own population. However, these same authors believe that the more researchers use similar measures, the more similar their findings are likely to be.

Weiss (1993) writes that for PTSD research, the use of a structured clinical interview is "highly desirable if not an absolute requirement" (p. 180). Furthermore, the use of a structured clinical interview insures that all PTSD diagnostic criteria are adequately covered. Weiss reports that reviews of research conducted without the use of a structured clinical interview have shown that important symptoms were left out in many of the studies. Hodgins (1995) has also stated that researchers and administrators need to use in-depth assessment techniques, especially structured clinical interviews, to determine the level of mental illness in a given inmate population; and Ogloff et al. (1993) have appealed for the screening of all inmates for mental illness.

The current research uses both self-report measures (the P-K Scale from the MMPI-2 and the Posttraumatic Stress Diagnostic Scale ${ }^{\mathrm{TM}}$ ) and a structured clinical interview (the Clinician-Administered PTSD Scale - DX) to assess for PTSD. This allows for a comparison between the two ways of determining the presence or absence of PTSD. In addition, an objective of this research is to provide BOP psychologists with different levels of PTSD assessment that can be used for screening and diagnosis.

\section{Participants}

Participants for this research were selected from inmates at the minimum security Federal Prison Camp (Camp) and medium security Federal Correctional Institution (FCI) in Cumberland, Maryland. Both of these facilities are exclusively for males. Fifty inmates were randomly selected from each facilities, for a total of 100 participants. 


\section{Power Analysis}

According to Keppel (1991), a study's power is the "probability of rejecting the null hypothesis when the alternative hypothesis is true" (p. 55). This means that a study's power is its ability to detect a treatment effect. For the purposes of this study, power is (a) the ability to identify an accurate prevalence rate of PTSD among all prison inmates assessed; and (b) the ability to identify accurate PTSD prevalence rates for inmates in the minimum and medium security facilities. The null hypothesis for (a) holds that there is no difference between prevalence rates for PTSD for males in the general population and male inmates, while the alternative hypothesis holds that such a difference exists. The null hypothesis for (b) holds that there is no difference between prevalence rates for prisoners in medium and minimum security facilities, while the alternative hypothesis holds that such a difference exists.

Keppel (1991) states that a power level of .80 is accepted as sufficient by most researchers. To determine what sample size allowed the attainment this level of power, the researcher used a formula provided by Howell (1992) and assumed a medium effect size of .50. Given these assumptions, an overall sample size of 100 has a power of .99 at a significance level of .01 . When one attempts to compare prevalence rates for medium and minimum security levels with sample sizes of 50 each, power drops to .71 at a significance level of .05 . However, as the Howell formula used to compute power assumes a two-tailed t-test, sample sizes of 50 should be adequate when, as in this case, the direction of difference has been predicted. Therefore, subset sample sizes of 50 each are sufficient for detecting PTSD prevalence differences between Camp and FCI inmates, and a total sample size of 100 is adequate for detecting prevalence differences between inmates and the general population.

\section{Materials}

Kulka et al. (1991) recommend the use of multiple assessment measures to ensure an accurate estimate of the rate of PTSD within a population. However, what Kulka et al. include under "multiple instruments" are not simply self-reports or structured interviews, but behavioral evaluations by the clinician and family members. Although such an extensive design is beyond the scope of this study, the researcher did use multiple 
measures of PTSD, including the Clinician-Administered PTSD Scale (CAPS-DX), the Posttraumatic Stress Diagnostic Scale ${ }^{\mathrm{TM}}$ (PDS ${ }^{\mathrm{TM}}$ ), and the P-K Scale of the MMPI-2 (PKS). The CAPS-DX includes a Life Events Checklist, that facilitated the gathering of information on the number of traumatic events experienced by inmates. In addition, three subtests (Information, Digit Span, and Picture Completion) from the Wechsler Adult Intelligence Scale-Revised (WAIS-R) were administered to estimate IQ, while the Hollingshead Four Factor Model (HFFI) will be utilized as a measure of childhood socioeconomic status.

Each participant was involved in two assessment sessions. In the first, a demographic questionnaire was completed by groups of participants immediately after they signed the informed consent form. This questionnaire included the HFFI factors. In addition, each participant completed the PKS, the PDSTM, and the Life Events Checklist. The time required for this first session was usually about one hour ( 15 minutes for an explanation of the project and the execution of the informed consent, and 45 minutes for the completion of the questionnaire, the PKS, and the PDS ${ }^{\mathrm{TM}}$ ). The second session was individual. In it, the WAIS-R subtests were administered, along with the CAPS-DX. This session lasted between 45 minutes and two hours (15 minutes to review any questions, 15 minutes for the WAIS-R, and a minimum of 15 minutes for the CAPS-DX). The following is a breakdown of the approximate administration time for each instrument: CAPS-DX (45-60 minutes, if PTSD present; 15 minutes, if not), PDS ${ }^{\mathrm{TM}}$ (15 minutes), and WAIS-R (15 minutes). A review of each assessment instrument follows.

\section{Clinician-Administered PTSD Scale (CAPS-DX)}

Development. The CAPS was developed by Blake et al. (1990) for use with male combat veterans. It has since been used with non-combatant males and females (Scotti, in press). The newest version of the instrument, the CAPS-DX, is tied directly to the DSMIV diagnostic criteria. The CAPS-DX is a structured clinical interview providing a more in-depth individual evaluation of PTSD symptoms than is offered by the PTSD modules of existing instruments, such as the Diagnostic Interview Schedule (DIS) or the Structured Clinical Interview for the DSM (SCID) (Blake et al., 1995). 
Administration and scoring. The CAPS-DX can be administered by trained clinicians or research assistants. If PTSD is present, the CAPS-DX takes approximately one hour to complete. If PTSD is not present, the CAPS-DX takes about 15 minutes to complete. The participant first completes a Life Events Checklist whose purpose is to determine whether or not the DSM-IV's (1994) Criterion A has been satisfied. The identified traumatic events are then used as reference points for symptom determination. The CAPS-DX itself consists of 30 items, all of which are scored on the basis of frequency and intensity of symptoms. Frequency is scored from $0-4$, with 0 being "never" and 4 being "daily (nightly) or almost every day (night)." Intensity is also scored from 0 4 , with 0 indicating "none" and 4 being "extreme, incapacitating distress." $A$ frequency rating of 1 or higher and an intensity rating of 2 or higher are required for the endorsement of any particular item. It should be noted that Weathers and Litz (1994) believe such a decision rule may lead to an overestimation of PTSD symptomatology. These authors suggest the Rule of Four as a more stringent scoring paradigm. According to this scoring procedure frequency plus intensity must total at least four before a symptom is considered endorsed. In both scoring systems, symptom endorsements are then counted toward the number required to fulfill Criterion B (reexperiencing), C (avoidance), and D (arousal) of the DSM-IV for a diagnosis of PTSD. The CAPS-DX provides a rating of global functioning which is also required for a PTSD diagnosis.

The clinician first assesses for current PTSD (that which has been present for at least the last month). If there is no current PTSD, the clinician assesses for lifetime PTSD, considering only those symptoms that occur within a single month during the participant's life. The clinician can derive either a continuous score or a dichotomous score for each individual question. If a continuous scores are chosen, their total can range from 0 to 136. By using a continuous score, the clinician can monitor the course of the disorder over time and estimate symptom severity (Scotti, in press). This study uses continuous scoring.

Blake et al. (1995) note that while the CAPS-DX may be the best structured interview currently available, it, too, has its drawbacks, including administration time, complexity, and scoring rules that have been rationally, rather than empirically derived. 
The authors also state that the instrument's psychometric properties may vary from population to population, and that the associated symptoms included in the interview may be less valuable with non-military participants.

Psychometric characteristics. Scotti (in press) reports that the CAPS-DX has high internal consistency (alpha of .76-.88) and inter-rater reliability (.84-1.0) with civilian and refugee populations. Newman et al. (1996) report that the convergent validity of the measure has been demonstrated via high correlations with self-reports such as the Mississippi Scale for Combat-Related PTSD (MSS) $(r=.91)$, the PKS $(r=.77)$, and structured interviews such as the SCID $(r=.89)$. Scotti notes that the CAPS's construct validity has been supported through work by Blanchard et al. (1994). Newman et al. report that the CAPS-DX has been demonstrated to have a sensitivity of 0.84 and a specificity of 0.95 .

\section{Keane PTSD Scale of the MMPI-2 (PKS)}

Development. Keane, Malloy, and Fairbank (1984) developed the PKS as a way of identifying individuals who are currently experiencing posttraumatic stress disorder. The scale was created by comparing the MMPI answers given by a sample of Vietnam veterans who had been clinically diagnosed with PTSD with those of a sample of veterans who had other diagnoses. Keane et al. (1984) identified 49 MMPI items for which the answers of the two groups were significantly different. The PK scale for the MMPI-2 consists of 46 items. Three of the original MMPI items were redundant and were dropped when the instrument was revised. Per Graham (1993), one of the existing items was also reworded, and per Allen (1994) the order of presentation of some items was changed.

As the MMPI-2 is now used in clinical practice, it makes sense to use the PKS from the newer instrument in any study that attempts to determine the prevalence of PTSD. However, because the original research on the use of the PKS was conducted using the original MMPI, it must be demonstrated that the versions are equally effective. Albrecht et al. (1994) administered both the MMPI and MMPI-2 to a sample of 47 Vietnam veterans with combat-related PTSD and found a high degree of overall congruence between the two instruments concerning the usual 2-8/8-2 PTSD profile. This finding was consistent with earlier results obtained by Ben-Porath and Butcher (1989) and 
Litz et al. (1991). However, Albrecht et al. found that PKS scores on the MMPI-2 were somewhat higher than those obtained using the MMPI. Building on work by Lyons and Scotti (1994) for the original MMPI, Herman, Weathers, Litz, and Keane (1996) looked specifically at the psychometric properties of the MMPI-2 PKS for both the embedded and stand-alone versions and examined its convergent validity with the CAPS and the MSS. As will be discussed below, the Herman et al. (1996) results appear solid enough to permit the use of the PKS from the MMPI-2 in the current study.

Administration and scoring. Raw scores (as opposed to t scores) are used to evaluate an individual's performance on the PKS. Herman et al. (1996) noted that a raw cut-off score of 24 for the 46 -item version resulted in sensitivity of .82 and specificity of .76 for the stand-alone version. For the embedded version, Herman et al. found that a raw cut-off score of 26 resulted in sensitivity of .72 and specificity of .82 . Both of these cutoffs are lower than the raw score of 30 determined by Lyons and Scotti (1994) for the 49item embedded and stand-alone versions of the PKS for the MMPI and the rationally derived cut-offs of 28 for the MMPI- 2 embedded version proposed by Lyons and Keane (1992). It should also be noted that the Herman et al. sample involved only Vietnam veterans.

Fairbank, McCaffrey, and Keane (1985) have indicated that the PKS may be susceptible to exaggeration by those wishing to appear worse off than they are. Others believe that high PKS scores are not indicative of "faking bad," and are simply part of a pattern of over-responding to the world (Allen, 1994). Whether or not the PKS can be "faked" and whether or not there is incentive to do so are questions of some debate. In any event, it seems clear that the PKS is most helpful when used in conjunction with other assessment instruments, especially a structured clinical interview.

Sometimes the MMPI-2 F scale is used to confirm an attempt to fake bad, with a T score of 88 being used as a cut-off. However, Graham (1993) states that very high T scores may be typical for PTSD patients. Allen (1994) also indicates that high F scale scores need to be interpreted with caution and are not necessarily indicative of an invalid profile when the test-taker is PTSD-positive. This seems to indicate, once again, that 
"faking bad" needs to be determined via a clinical examination for PTSD and a clinician's professional evaluation of the subject rather than psychometric assessment.

Psychometric characteristics. Graham (1993) reports that when the PKS for the MMPI and the MMPI-2 were compared, their correlation was .80. Furthermore, internal consistency for the MMPI-2 PKS was .85 and .87 for men and women, respectively, while test-retest reliability was .86 and .89 , respectively. Herman et al. (1996) identified a strong Pearson correlation $(\underline{r}=.90)$ between the embedded and stand-alone versions of the MMPI-2 PKS and a solid test-re-test reliability for the stand-alone version $(\underline{r}=.95)$.

Despite the fact that the PKS has been rigorously validated, there appears to be room for improvement in the area of construct validity. Graham (1993) states that the scale "is suggestive of great emotional turmoil" (p. 162), as indicated by the following problems: Anxiety, sleep disturbance, guilt, depression, disturbing thoughts, lack of emotional control, and feeling misunderstood and mistreated. After reviewing the MMPI2 items which make up the PKS, I believe they thoroughly address only one of the major PTSD problem areas identified by van der Kolk, Pelcovitz, Roth, Mandel, McFarlane, and Herman (1996), that being affective dysregulation, while failing to include a sufficient number of items to allow a comprehensive evaluation of the second and third (dissociation and somatization) areas. In addition, as Foa (1995) points out, instruments such as the PKS are empirically derived and, therefore, not based on the DSM criteria for PTSD. This means the PKS is not a true diagnostic tool and is better used as an initial screen or for secondary confirmation.

Graham (1993) also cites a number studies subsequent to the original Keane et al. (1984) study that looked at the scale's discriminant validity. Although the studies showed that the scale consistently discriminated between PTSD positive and negative Vietnam veterans, the cut-off range varied widely (from 8.5 to 30). Newman et al. (1996) report that this may be an indication of the need to establish cut-off scores for separate populations. Herman et al. (1996) agree whole-heartedly with this, stating that cut-off scores are based on the base rate in any given population. They note that this base rate is subject to fluctuation and that local cut-offs need to be established whenever possible. Graham also reports that the PKS does a better job of distinguishing between individuals 
when no other diagnosis is present. However, as we have seen, PTSD carries with it a high degree of comorbidity and this is a further complicating factor.

Convergent validity has been assessed by a number of studies (Graham, 1993). In these, it has been found that scores on the PKS are highly correlated with the results from structured clinical interviews for PTSD. Herman et al. (1996) provided Pearson correlations for the embedded and stand-alone versions of the MMPI-2 PKS with both the MSS and the CAPS. For the Mississippi Scale, the correlation was .81 for the embedded format and .85 for the stand-alone format. For the CAPS-DX, the correlation was .77 for the embedded format and .80 for the stand-alone format.

Although most of the studies on the PKS have been conducted using combatexposed populations, some studies (Koretzky \& Peck, 1990; McCaffrey, Hickling, \& Marrazo, 1989; Sloan, 1988) indicate that the PKS scale also can be used effectively with civilian populations. This statement needs to be expanded by the caveat that civilians seem to have lower mean PKS scores than do combat veterans. Therefore, as discussed above, cut-offs may vary from group to group and previously established cut-offs should be used with caution.

Despite the fact that there are some potential problems associated with using the PKS, its questions are dissimilar from those contained in the other assessment measures and are not linked directly to the DSM criteria. These facts allow for a kind of alternative assessment of the presence of PTSD symptomatology among inmates. If the results of the stand-alone version of the PKS are highly correlated with the results of the CAPS-DX, then this is an indication that the PKS can be used as a screen for inmates with PTSD.

\section{Posttraumatic Stress Diagnostic Scale ${ }^{\text {TM }}$ (PDSTM)}

Development. The PDS ${ }^{\mathrm{TM}}$ was developed by Foa (1995). It is designed to address some of the weaknesses of self-reports for PTSD, including the fact that they are usually normed for specific populations and that they are empirically, as opposed to theoretically, derived (Foa, Cashman, Jaycox, \& Perry, 1997). As we have already seen, when instruments are normed on specific populations, it makes the use of cut-off scores questionable when different populations are assessed with the same instrument. In addition, the validity of empirically-derived instruments such as the MSS and the PKS 
may be a function of those same samples, and it may be difficult to generalize to other populations. Another potential problem with self-report scales is that the items they use do not always comprehensively reflect the DSM criteria for the disorder. Therefore, Foa set about constructing an assessment instrument that would meet these concerns and conform to the PTSD diagnostic criteria as presented in the DSM-IV (1994). The PDS ${ }^{\mathrm{TM}}$ was validated on a sample $(\underline{N}=248)$ of individuals who ranged in age from $18-65$, with a mean of 38.6 years. Males made up $54.8 \%$ of the sample. Racially, the sample was $64.9 \%$ Caucasian and $31 \%$ African American. In terms of education, $15.7 \%$ of the sample had not graduated high school, $27.4 \%$ had, and $56.9 \%$ had at least some college or beyond.

Administration and scoring. The PDSTM is a 49-item self-report. It takes approximately 15 minutes to administer and is geared to an individual with an eighthgrade reading level. A PTSD diagnosis is given if and only if all six DSM-IV (1994)criteria are endorsed. The first part of the PDSTM includes 13 items and is concerned with the type of trauma experienced. For the purposes of the proposed research, it is especially interesting to note that the PDSTM includes imprisonment as one of the stressors. Part 2 (questions 14-21) concerns itself with the most troubling of all traumatic events checked: when the event took place, whether it was perceived to be lifethreatening, and whether or not it precipitated a feeling of helplessness in the victim. Part 3 (questions 22-40) addresses the remaining criteria and duration. Part 4 (questions 4149) assesses global functioning.

The PDSTM also provides a Symptom Severity Score (SSS) which ranges from 051. The score is obtained by adding the scores $(0=$ "not at all or only once," $1=$ "once a week or less," 2 = "2-4 times a week," and 3 = "5 or more times a week") achieved for items 22-38. Table 5 provides an overview of the levels of PTSD severity as determined by the PDS ${ }^{\mathrm{TM}}$. Unfortunately, the cut-offs for severity were validated on independent samples that were not part of the normative sample. Instead, the responses of 280 female assault victims were used to determine cut-offs. Therefore, they may not be useful with other populations.

An estimated level of impairment is also provided based on the respondent's answers to Part 4: Global Functioning (questions 41-49). This is summarized in Table 6. 
Table 5

PDSTM Severity Levels.

Score Designation

\begin{tabular}{ll}
\hline$\leq 10$ & Mild \\
$\geq 11$ and $\leq 20$ & Moderate \\
$\geq 21$ and $\leq 35$ & Moderate to Severe \\
$\geq 36$ & Severe \\
\hline
\end{tabular}

Note. Adapted from Foa (1995).

Table 6

PDSTM Estimated Levels of Impairment.

\begin{tabular}{ll} 
Number of yes responses & Impairment \\
\hline 0 & None \\
$1-2$ & Mild \\
$3-6$ & Moderate \\
$7-8$ or yes to item 49 & Severe \\
\hline
\end{tabular}

Note. Adapted from Foa (1995). 
The PDS ${ }^{\mathrm{TM}}$ also allows differentiation between acute, chronic, and delayed onset PTSD to be made depending on answers to questions 39 and 40 , which concern the length of time symptoms have been experienced and how soon after a traumatic event they appeared.

Psychometric characteristics. In normative studies conducted by Foa (1995), the

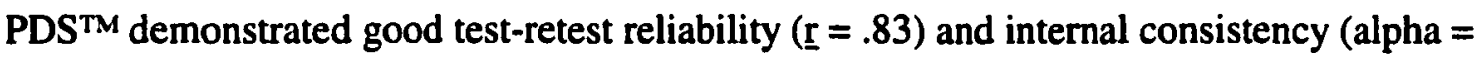
$.92)$. Concurrent validity as measured against the SCID was good (kappa $=.59)$, with an agreement of $79.4 \%$ between the two instruments. These same studies showed the PDS ${ }^{\mathrm{TM}}$ to have a sensitivity of 0.82 and a specificity of 0.77 . As there are no other existing selfreports for the DSM-IV (1994) diagnosis of PTSD, measures of convergent validity concerned themselves with the SSS and other instruments such as the Beck Depression Inventory, the State/Trait Anxiety Index, and the Impact of Events Scale. All of these comparisons showed good convergent validity. As with the PKS, a high correlation between the PDSTM and the CAPS-DX for an inmate population would be a good indication that the PDS ${ }^{\mathrm{TM}}$ can be used as an effective PTSD screening tool.

\section{The Hollingshead Four Factor Index of Social Status (HFFD}

Development. The HFFI was first described in a 1975 unpublished article by August B. Hollingshead of Yale University. It treats social status as a multidimensional concept based on three assumptions: (a) that an inequality of status exists; (b) that the most important factors involved in status are occupation, education, sex, and marital status; and (c) that the evaluation and combination of these factors can permit the estimation of an individual's status position in the society.

Administration and scoring. The factors that are scored are occupation and education. For occupation, the scores can range from 1-9. For education, the scores can range from 1-7. For determination of the occupational score, the researcher refers to categories provided by Hollingshead. For determination of the educational score, the researcher assigns scores as indicated in Table 7.

Occupational scores are multiplied by a factor of 5 and educational scores by a factor of 3 . The scores of married couples are summed and divided by 2 . If a couple is divorced and the spouse with child custody works, then the score of that spouse alone is 
Table 7

Hollingshead's (1975) Educational Scores

\begin{tabular}{ll} 
Score & Education level \\
\hline 1 & Less than 7th grade education \\
2 & Completed 9th grade \\
3 & Completed 10th or 11th grade \\
4 & High school graduate \\
5 & Completed one year of college or specialized training \\
6 & Graduated from college \\
7 & Obtained a graduate degree \\
\hline
\end{tabular}

Table 8

Hollingshead's Social Stratum and Score Range for the HFFI

Social stratum

Score range

Major business/professional

$66-55$

Medium business/minor professional

$54-40$

Skilled craftsman, clerical, sales

$39-30$

Machine operator. semiskilled workers

$29 \cdot 20$

Unskilled laborers, menial service workers

$19-8$ 
used to compute social status. If a couple is divorced and the spouse with custody is supported by his or her former spouse, then the score of the former spouse alone is used to compute social status. The maximum score attainable is 66 . The relation between social status scores and social classes as adapted from Hollingshead (unpublished manuscript, p. 23) are summarized in Table 8.

Psychometric characteristics. In Hollingshead's original two-factor model, there was a correlation between social status and occupation and education of $\underline{r}=0.975$. Comparing the prestige of occupations as determined in a study by the National Opinion Research Center with his own nine occupational categories, Hollingshead came up with an $\underline{r}=.927$. Hollingshead assumes that education and occupation are positively correlated.

\section{Wechsler Adult Intelligence Scale-Revised (WAIS-R)}

Development. The WAIS-R was developed by Wechsler (1981) to measure "overall capacity for intelligent behavior" (p. 7). It makes use of IQ scores and their percentile rankings to compare the results of individuals within and between specific age groups. If two individuals in different age groups have the same IQ, then we know that their relative standing within their specific groups is the same. The WAIS-R consists of 11 subtests. Six of the subtests measure verbal ability and five measure performance ability. In addition to computation of the Full Scale IQ, this allows the derivation Verbal and Performance IQ's. Full Scale, Verbal, and Performance IQ scores have a mean of 100 and a standard deviation of 15 .

Although the WAIS-R has, as noted below, excellent psychometric characteristics, its major drawback is its administration time. For adults, the assessment can easily take one and one-half hours, and for this reason the WAIS-R is not well-suited to research. For that reason, Kaufman, Ishikuma, and Kaufman-Packer (1991) examined dyads, triads, and tetrads of WAIS-R subtests to determine if one could substantially reduce administration time without losing too much of the WAIS-R's reliability and validity. To do this, Kaufman et al. (1991) utilized the WAIS-R's standardization sample results and computed correlations between the full WAIS-R and a variety of subtest dyads, triads and tetrads. In doing so, the authors also considered the placement of the 
subtests and how that might effect results. What Kaufman et al. discovered was that the subtest constellations with the best psychometric properties and the shortest administration times were Information and Picture Completion (12 minutes administration time), Information, Digit Span and Picture Completion (16 minutes), and Arithmetic, Similarities, Picture Completion, and Digit Symbol (19 minutes). For this study, the author has chosen to use the triad to assess Full Scale intelligence.

Administration and scoring. The WAIS-R can be given to subjects age 16 and older. The subtests are given in a prescribed order, with Information, Picture Completion, and Digit Span being the first three subtests administered. Raw scores for each subtest are computed and transformed into scaled scores with a mean of 10 and a standard deviation of three. These scaled scores are then used to compute IQ's. IQ levels are used to help interpret the results, as delineated in Table 9.

When using the WAIS-R Triad suggested by Kaufman et al. (1991), the subtests are administered in precisely the same way as one would administer them during a full WAIS-R. The only difference is in how the IQ score is derived. Subtests are first scored as usual. However, instead of converting the raw scores to regular scaled scores, they are converted to age-corrected scaled scores and the three results are summed. This sum is then plugged into a general formula to compute the Full Scale IQ.

Psychometric characteristics. Reliability of the WAIS-R was computed using both split-half and test-retest methods, with all subtests except Digit Symbol and Digit Span undergoing the former procedure. Reliability coefficients were computed for all nine age groups and an average taken. For the Full Scale IQ, the average reliability was .97 . The validity of an IQ assessment is much more difficult and controversial subject. However, Kaufman (1990) stated that "in essence...the IQ construct, as measured by contemporary intelligence tests, is valid when defined within the societal context and when the IQ's limitations are kept fully in mind" (p. 18). Kaufman then goes on to demonstrate the relation that exists between IQ and academic achievement, education level, occupation, and job performance. Wechsler (1981) also notes that a relation exists between IQ and prenatal factors, nutrition, mental retardation, and income. These are all reflections of criterion-related validity. According to Anastasi (1988), Wechsler also emphasized the 


\section{Table 9}

Interpretation of WAIS-R IQ Levels

IQ score Interpretation

69 and below Mentally retarded

$70-79 \quad$ Borderline retarded

$80-89 \quad$ Low or dull normal

$90-110 \quad$ Normal

$111-120 \quad$ High or bright normal

$121-130 \quad$ Superior

130 and up Very superior

Note. Adapted from Kaufman (1990). 
content validity of his assessment, noting that the abilities measured by his subtests had been used successfully in other tests of intelligence and had proven to be of clinical value. Anastasi writes that construct validity may be inferred from the average intercorrelations of the WAIS-R Full Scale IQ with each subtest. These ranged from a low of .64 to a high of .85 . Finally, a measure of validity may be obtained by correlating the results of tests purported to measure the same construct. When this was done for the Stanford-Binet and the WAIS, the correlation was .85 (Wechsler, 1981).

By comparison with the full WAIS-R, Kaufman's Triad had a mean reliability coefficient of .91 . Validity was measured by determining how well the brief test compared with the full test. The mean validity for the Kaufman Triad was .92 . 


\section{Chapter 4: RESULTS}

\section{Introduction}

\section{The Research Questions}

The basic research questions this study set out to answer are as follows: Are lifetime and current posttraumatic stress disorder (PTSD) rates higher among male federal prison inmates than among men in the general population? Do lifetime and current PTSD rates increase as the prison security level increases? Are men from minority groups, men with lower global IQ scores, or men who grew up in families of lower socioeconomic status (SES) more likely to have PTSD than men who are white, who have higher global IQ scores, or men from higher SES families? Can the incarceration process itself be a PTSD precipitant? And, finally, are prison authorities aware of the number of men under their control who have or have had PTSD? The information that follows will help us answer those questions.

\section{Chapter Layout}

This chapter provides information related to the research questions listed above. It begins by describing the research setting and continues on to compare the population of this site with the population of all inmates under the control of the Federal Bureau of Prisons (BOP). Next, the selection of the sample is described, along with its characteristics. Once this has been accomplished, the question of potentially traumatic stressors experienced by the participants is addressed. It makes sense to discuss stressors first, because without the presence of a stressor that meets the requirements of Citerion $\mathrm{A}$ of the DSM-IV (1994) diagnosis for PTSD (see Appendix I), the disorder cannot be identified as such. Once the stressors have been reviewed, the lifetime and current prevalence rates of PTSD identified by the study are presented along with the performance of the three diagnostic instruments used. This is followed by the presentation of the results of a multiple linear regression analysis using Clinician-Administered PTSD Scale scores as the dependent variable. Finally, each research hypothesis is examined based on the results presented.

It may be helpful for the reader to know in advance that the study found no difference in prevalence rates between inmates in minimum and medium security. 
Therefore, participants in the two security levels were combined to compute lifetime and current PTSD prevalence rates. The former was $30 \%$ (30 of 100 participants had had PTSD at some time during their lives, including at the time of the study) and the latter was $14 \%$ (14 of 100 participants were identified as having PTSD at the time of the study). The Research Setting

Data collection sites. Data collection for this study was conducted during the period March 23 to June 17, 1998. Data on inmates detained in a minimum security facility were collected at the Federal Prison Camp (FPC) in Cumberland, MD, and data on inmates detained in a medium security facility were collected at the Federal Correctional Institution (FCI) in the same city. FPC and FCI Cumberland are located on the same campus and are part of the Federal Bureau of Prison's (BOP) Mid-Atlantic Region. They draw most of their inmates from that geographic area.

The Research Sample

\section{Derivation and Characteristics}

Inmate populations. As of September 30,1995, the BOP was responsible for 100,958 inmates held in its facilities nationwide (US Department of Justice, 1995a). Approximately $86 \%$ of these individuals are incarcerated in institutions with a security level of medium or less, and $92 \%$ are male. During this study, the number of inmates at FPC and FCI Cumberland totaled 1,260 (273 at the FPC and 987 at the FCI). All of these inmates were male. The racial composition of the national and sample populations are provided in Table 10. Following BOP custom, Hispanics have not been classified as a separate race and are included in the black and white categories, as appropriate.

Chi-square analyses comparing the number of blacks and whites in the sample with the number of blacks and whites incarcerated nationally and at Cumberland indicated that the sample is not reflective of either the racial composition of the federal system as a whole or the racial composition of the Cumberland site itself. The sample had more blacks and fewer whites than the federal system, and fewer blacks and more whites than the Cumberland site as a whole. However, the Cumberland facility's racial composition also varies significantly from that of the federal system, having more blacks and fewer whites. A summary of these analyses is presented in Table 11. 
Table 10

Racial Composition: Federal Prison System, Sample, and FPC and FCI Cumberland

\begin{tabular}{lrlll}
\hline Group & $\begin{array}{l}\text { Number } \\
\text { of inmates }\end{array}$ & $\begin{array}{l}\text { Percent } \\
\text { black }\end{array}$ & $\begin{array}{l}\text { Percent } \\
\text { white }\end{array}$ & $\begin{array}{l}\text { Percent } \\
\text { other }\end{array}$ \\
\hline Federal system & 103,398 & $41 \%$ & $56 \%$ & $3 \%$ \\
FPC/FCI Cumberland & 1,260 & $64 \%$ & $35 \%$ & $1 \%$ \\
Total sample & 100 & $54 \%$ & $44 \%$ & $2 \%$ \\
FPC sample & 50 & $42 \%$ & $58 \%$ & $0 \%$ \\
FCI sample & 50 & $66 \%$ & $30 \%$ & $4 \%$ \\
& & & & \\
\hline
\end{tabular}

Table 11

Chi-Square Comparisons of Black and White Populations

Comparison df $\quad$ N $\chi^{2}$

$\begin{array}{llll}\text { National vs. Cumberland } & 1 & 99 & 20.32^{*} \\ \text { Cumberland vs. sample } & 1 & 99 & 20.84^{*} \\ \text { FPC vs. FCI } & 1 & 98 & 33.04^{*}\end{array}$

${ }^{*} \mathrm{p}<.0001$ 
Table 12

Sample Recruitment: Distribution of Participants and Non-participants

\begin{tabular}{lccc}
\hline Sample segment & $\begin{array}{l}\text { Number } \\
\text { accepting }\end{array}$ & $\begin{array}{l}\text { Number } \\
\text { declining }\end{array}$ & $\begin{array}{l}\text { Percent } \\
\text { accepting }\end{array}$ \\
\hline Total sample & 100 & 88 & $53 \%$ \\
FPC sample & 50 & 40 & $56 \%$ \\
FCI sample & 50 & 48 & $51 \%$ \\
Blacks & 55 & 70 & $44 \%$ \\
Whites & 43 & 18 & $71 \%$ \\
Others & 2 & 0 & $100 \%$ \\
& & & \\
\hline
\end{tabular}


How the sample was determined. The researcher was provided with an alphabetical listing of inmates at each site which he numbered sequentially. These lists were then compared with random number tables (Kirk, 1995) to create a pool of potential participants. Inmates were called to meetings in groups of 8-10 in the order in which they were drawn. The project was explained to them by the researcher; they were given an opportunity to inspect all research materials; and were asked if they wanted to participate or not. Those who agreed to participate were then asked to sign an informed consent. Acceptance rates for the total sample, for each security level, and for racial groups are provided in Table 12.

Teplin $(1990,1996)$ reported participation rates of $95.4 \%$ and $95.8 \%$, respectively, in two prison studies she conducted. In the former study, male inmates were paid $\$ 5.00$, and in the latter, female inmates were paid $\$ 15.00$ for their participation. Neither of these studies involved federal inmates. In their study of inmates in penitentiaries in Quebec, Hodgins and Cote (1990) had a participation rate of $78 \%$. These researchers did not pay their participants. H. Lebowitz (personal communication, August 27, 1998) of the BOP's Research Office indicated that while the BOP does not keep official records on research participation rates, anecdotal reports of colleagues suggested rates of $55-80 \%$ for "nonthreatening" studies.

Characteristics of the sample. As has been mentioned, $54 \%$ of the study's sample was black, $44 \%$ white, and $2 \%$ Asian and Native American. However, the racial distributions differed by security level, as is shown in Table 11. Inmate age for the entire sample ranged from 22 to 69 , with a mean of $37.9(\underline{S D}=10.4)$. This mean did not differ from that of the federal system, as a whole. A $2 \times 2$ analysis of variance (ANOVA), using security and race as the general factors, indicated that a significant interaction was present for this variable, $\underline{F}(1.94)=11.3, \mathrm{p}<.001$. The mean ages for blacks and whites in minimum security were $37.7(S D=9.4)$ and $45.3(S D=10.1)$, respectively. This difference was significant. The mean ages for blacks and whites in medium security were $34.1(\mathrm{SD}=8.6)$ and $31.5(\mathrm{SD}=5.9)$, respectively. This difference was not significant. The mean global IQ of the entire sample, as determined by the administration of three subtests of the WAIS-R, was $95.4(\underline{\mathrm{SD}}=13.0)$. A $2 \times 2$ ANOVA was also conducted for 
this variable, using the same general factors cited earlier. The ANOVA indicated that although no interaction was present, there was a main effect for race, $F(1,93)=10.5, \mathrm{D}<$ .01 , with whites $(\underline{M}=100.8, \underline{S D}=11.4)$ scoring higher than blacks $(\underline{M}=91.4, \underline{S D}=12.7)$. Finally, the overall sample averaged $34.2(S D=13.98)$ on the continuous measure of childhood socioeconomic (SES) status, with the same $2 \times 2$ ANOVA producing neither an interaction nor main effects for this variable. Means for age, IQ, and childhood SES by security level and race are provided in Tables 13 and 14. Mean global IQ and SES scores were not available for the federal system, as a whole, and could not be compared with the figures derived from this sample. Neither age, nor IQ, nor childhood SES were significantly different for inmates with and without PTSD (Table 15).

A visual inspection of stem-and-leaf plots for the variables age, IQ, and SES indicated that these variables were not normally distributed. This was confirmed by the Kolmogorov-Smirnov tests, utilizing the Lilliefors correction (K-S Lilliefors). The results for these tests were $\underline{\mathrm{K}-\mathrm{S}}=.11, \mathrm{p}<.01$ for age; $\underline{\mathrm{K}-\mathrm{S}}=.09, \mathrm{p}<.03$ for $\mathrm{IQ}$; and $\underline{\mathrm{K}-\mathrm{S}}=.13, \mathrm{p}$ $<.001$ for SES. However, Howell (1992) has stated that ANOVA's are robust enough to withstand a violation of the normality assumption.

In summary, the sample's racial composition differed significantly from that of both the federal system, in general, and the Cumberland facility, in particular, while the sample's mean age was consistent with the average age of all federal prisoners. Unfortunately, the mean IQ score and childhood SES rating for the sample could not be compared with the federal system as a whole. When age, IQ, and childhood SES were compared by security level and for blacks and whites, there was an interaction and a main effect for security level for the first, a main effect for race the second, and neither an interaction nor a main effect for the third. Inmates with and without PTSD did not have significantly different scores on any of these variables. 
Table 13

Age, IQ, and SES Means by Security Level

Variable Minimum $\underline{M}(\underline{S D}) \quad$ Medium $\underline{M}(\underline{S D})$

\begin{tabular}{lll}
\hline Age & $42.1(10.4)^{* *}$ & $33.6(8.4)^{* *}$ \\
IQ & $98.3(14.6)^{*}$ & $92.4(10.3)^{*}$ \\
SES & $35.6(16.0)$ & $33.2(11.6)$ \\
\hline
\end{tabular}

${ }^{*} \mathrm{p}<.05 * * \mathrm{p}<.001$

Table 14

Age, IQ, and SES Means for Blacks and Whites

\begin{tabular}{llc}
\hline Variable & Black $\underline{\mathrm{M}}(\underline{\mathrm{SD}})$ & White $\underline{\mathrm{M}}(\underline{\mathrm{SD}})$ \\
\hline Age & $35.5(9.0)^{*}$ & $40.6(11.0)^{*}$ \\
IQ & $91.4(12.7)^{* *}$ & $100.8(11.4)^{* *}$ \\
SES & $34.3(13.0)$ & $34.4(15.1)$ \\
\hline
\end{tabular}

${ }^{*} \mathrm{p}<.05 * * \mathrm{p}<.001$

Table 15

Age, IQ and SES Means for Inmates with and without PTSD

\begin{tabular}{lll}
\hline & $\begin{array}{l}\text { PTSD Positive Inmates } \\
\text { Variable }\end{array}$ & $\underline{\mathrm{M}(\underline{\mathrm{SD}})}$ \\
& & $\underline{\mathrm{M}}(\underline{\mathrm{SD}})$ \\
\hline Age & $36.2(10.4)$ & $38.6(10.4)$ \\
IQ & $95.6(11.9)$ & $95.3(13.5)$ \\
SES & $33.1(13.5)$ & $35.1(14.3)$ \\
\hline
\end{tabular}


Results

Potentially Traumatic Stressors Experienced by the Sample

Potentially traumatic stressors at the research sites. Psychology staff at the research sites indicated they knew of no events that had taken place during the year prior to data collection that might have been potentially traumatic for large numbers of inmates (e.g., natural disasters, fires, completed suicides, serious physical assaults, murders, or riots).

Number of potentially traumatic stressors experienced by participants. The CAPSDX and the PDSTM provide an opportunity for individuals to identify potentially traumatic stressors experienced during a lifetime. The CAPS-DX lists 18 possible stressors, each of which can be experienced, witnessed, or learned about. The PDSTM lists 12 potential stressors and limits the response to those that have been experienced or witnessed. A category for "any other stressful event" is included in the totals given for both instruments. Therefore, a participant could theoretically endorse 54 potential

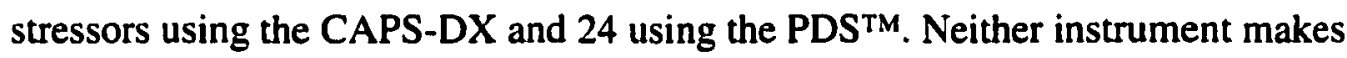
allowance for a participant's having experienced multiple stressors of the same type (e.g., multiple motor vehicle accidents). The number of stressors identified by participants using the CAPS-DX ranged from 1-30, while the range for the PDSTM was 1-10. The CAPS-DX resulted in a mean of $8.7(\underline{S D}=5.6)$ stressors reported per participant and the PDS $^{\mathrm{TM}}$ resulted in a mean of $3.8(\underline{\mathrm{SD}}=1.9)$. The mean number of stressors reported by different sample groups and their t-test significance levels are displayed in Table 16.

Type of traumatic stressors identified to satisfy Criterion A. Of the 100 men in the sample, 15 had not experienced a stressor that met the requirements of Criterion $A$ of the DSM-IV (1994) diagnosis for PTSD (see Appendix I). Ten of these 15 men were white. In response to the CAPS-DX interview, 11 different stressors satisfying Criterion $\mathrm{A}$ were identified by the men in the sample, and four of these stressors accounted for $61 \%$ of the cases. The top four stressors were assault with a weapon; the sudden, unexpected death of someone close; incarceration; and motor vehicle accidents (Table 17). 
Table 16

Mean Number of Potential Stressors Identified by CAPS-DX and PDSTM

\begin{tabular}{lcll}
\hline Sample Portion & $\underline{\mathrm{N}}$ & CAPS-DX $\underline{\mathrm{M}}$ (SD) & PDS $^{\mathrm{TM}} \underline{\mathrm{M}}$ (SD) \\
\hline Full sample & 100 & $8.7(5.6)$ & $3.8(1.9)$ \\
Blacks & 54 & $8.8(5.1)$ & $3.7(1.8)$ \\
Whites & 44 & $8.9(6.2)$ & $4.0(2.2)$ \\
Minimum security & 50 & $8.1(5.5)$ & $3.5(1.9)$ \\
Medium security & 50 & $9.3(5.7)$ & $4.2(2.0)$ \\
Blacks in minimum security & 42 & $8.9(5.2)$ & $3.3(1.2)$ \\
Whites in minimum security & 58 & $7.6(5.7)$ & $3.7(2.2)$ \\
Blacks in medium security & 66 & $8.7(5.2)$ & $4.0(2.0)$ \\
Whites in medium security & 30 & $11.3(6.6)$ & $4.6(2.1)$ \\
Inmates with life PTSD & 30 & $12.4(6.7)^{*}$ & $4.9(2.3)^{* *}$ \\
Inmates w/o life PTSD & 70 & $7.6(4.1)^{*}$ & $3.4(1.6)^{* *}$ \\
Blacks with life PTSD & 12 & $13.2(7.4)^{*}$ & $5.0(2.2)^{* * *}$ \\
Blacks w/o life PTSD & 42 & $7.5(3.4)^{*}$ & $3.4(1.5)^{* * *}$ \\
Whites with life PTSD & 18 & $12.0(6.7)^{*}$ & $4.8(2.9)^{* * *}$ \\
Whites w/o life PTSD & 26 & $6.7(5.0)^{*}$ & $3.9(1.7)^{* * *}$ \\
\hline
\end{tabular}

Note. Life $=$ lifetime. $w / 0=$ without.

${ }^{*} \mathrm{p}<.001$

$* * \mathrm{p}<.01$

$* * * \mathrm{~g}<.05$ 
Table 17

Stressors Identified to Satisfy Criterion A: Totals by Sample, Security Level, and Race

\begin{tabular}{|c|c|c|c|c|c|c|}
\hline \multirow[t]{2}{*}{ Stressor category } & \multirow[t]{2}{*}{$\underline{\mathbf{N}}$} & \multicolumn{2}{|c|}{ Security Level } & \multicolumn{3}{|l|}{$\underline{\text { Race }}$} \\
\hline & & Min. & Med. & Black & White & Other \\
\hline Assaulted with weapon & 18 & 5 & 13 & 13 & 5 & 0 \\
\hline S.U.D. & 17 & 10 & 7 & 12 & 5 & 0 \\
\hline Incarceration & 15 & 8 & 7 & 7 & 8 & 0 \\
\hline None & 15 & 7 & 8 & 5 & 10 & 0 \\
\hline Motor vehicle accident & 11 & 4 & 7 & 5 & 5 & 1 \\
\hline Witnessed violent death & 6 & 3 & 3 & 1 & 4 & 1 \\
\hline Military combat & 6 & 6 & 0 & 4 & 2 & 0 \\
\hline Life-threatening illness & 6 & 4 & 2 & 4 & 2 & 0 \\
\hline Childhood sexual abuse & 2 & 2 & 0 & 0 & 2 & 0 \\
\hline Killed/harmed someone & 2 & 0 & 2 & 1 & 1 & 0 \\
\hline Accident at work & 1 & 1 & 0 & 1 & 0 & 0 \\
\hline Street combat & 1 & 0 & 1 & 1 & 0 & 0 \\
\hline Total & 100 & 50 & 50 & 54 & 44 & 2 \\
\hline
\end{tabular}

Note. $\underline{\mathbf{N}}=$ Number. Min $=$ Minimum Security. Med. $=$ Medium Security.

S.U.D. = Sudden, unexpected death of someone close. 


\section{Table 18}

Stressors Associated with Lifetime PTSD: Totals by Sample, Security Level, and Race

\begin{tabular}{lrrrrrr}
\hline Stressor category & $\underline{N}$ & \multicolumn{2}{l}{ Security Level } & & \multicolumn{2}{l}{ Race } \\
& & Min. & Med. & & Black White \\
& & & & & \\
\hline Assaulted with weapon & 4 & 0 & 4 & 1 & 3 \\
S.U.D. & 5 & 4 & 1 & 2 & 3 \\
Incarceration & 7 & 2 & 5 & 2 & 5 \\
Motor vehicle accident & 3 & 1 & 2 & 2 & 1 \\
Witnessed violent death & 2 & 0 & 2 & 0 & 2 \\
Military combat & 5 & 5 & 0 & 3 & 2 \\
Life-threatening illness & 1 & 1 & 0 & 0 & 1 \\
Killed/harmed someone & 1 & 0 & 1 & 0 & 1 \\
Accident at work & 1 & 1 & 0 & 1 & 0 \\
Street combat & 1 & 0 & 1 & 1 & 0 \\
Total & 30 & 14 & 16 & 12 & 18 \\
& & & & & \\
\hline
\end{tabular}

Note. $\underline{\mathbf{N}}=$ Number. Min $=$ Minimum Security. Med. $=$ Medium Security.

S.U.D. = Sudden, unexpected death of someone close. 
Criterion A stressors identified by inmates with lifetime PTSD. When stressors that satisfied Criterion A for the $\mathbf{3 0}$ men with lifetime PTSD are examined, the picture changes a bit. Ten stressors were associated with the cases of lifetime PTSD, with five of the 10 accounting for 24 of 30 cases, or $80 \%$. These five are incarceration; military combat; the sudden, unexpected death of someone close; assault with a weapon; and motor vehicle accidents. A summary of all stressors identified by inmates with lifetime PTSD broken down by security level and race is provided in Table 18.

Criterion A stressors identified by inmates with current PTSD. In this case, only seven stressors were associated with the 14 cases of current PTSD and three of them accounted for 10 of the cases ( $71 \%$ ). Those three were incarceration; assault with a weapon; and the sudden, unexpected death of someone close. Stressors associated with current PTSD and broken down by security level and race are presented in Table 19.

Percentage of stressors associated with lifetime and current PTSD. Seven of 11 stressor categories identified by participants as fulfilling Criterion $\mathrm{A}$ had a frequency of six or greater within the sample. Those categories and the percentages with which they resulted in a lifetime diagnosis of PTSD are provided in Table 20. When a stressor was identified as fulfilling the requirements of Criterion A, a lifetime PTSD diagnosis resulted $35 \%$ ( 30 of 85 cases) of the time.

In the case of current PTSD, only four of the stressors with a frequency of six or more resulted in at least one diagnosis. Those categories and the percentages with which they resulted in current PTSD are provided in Table 21. When a stressor was identified as fulfilling the requirements of Criterion A, a current PTSD diagnosis resulted $16 \%$ (14 of 85 cases) of the time.

Frequency of lifetime and current stressors by race. A recapitulation of part of the information provided in Tables 17 and 18 is provided in Table 22. This table highlights the frequency of identified stressors associated with lifetime and current PTSD among blacks and whites. 
Table 19

Stressors Associated with Current PTSD: Totals by Sample, Security Level, and Race

$\begin{array}{llll}\text { Stressor category } & \underline{\mathrm{N}} & \frac{\text { Security Level }}{\text { Min. Med. }} & \text { Bace } \\ & \text { Black White }\end{array}$

\begin{tabular}{lrrrrr}
\hline Assaulted with weapon & 2 & 0 & 2 & 0 & 2 \\
S.U.D. & 2 & 2 & 0 & 0 & 2 \\
Incarceration & 6 & 2 & 4 & 2 & 4 \\
Military combat & 1 & 1 & 0 & 1 & 0 \\
Killed/harmed someone & 1 & 0 & 1 & 0 & 1 \\
Accident at work & 1 & 1 & 0 & 1 & 0 \\
Street combat & 1 & 0 & 1 & 1 & 0 \\
Total & 14 & 6 & 8 & 5 & 9 \\
& & & & & \\
\hline
\end{tabular}

Note. $\underline{\mathbf{N}}=$ Number . Min $=$ Minimum Security. Med. $=$ Medium Security.

S.U.D. = Sudden, unexpected death of someone close. 
Table 20

Percentage of Stressor Category Resulting in Lifetime PTSD

\begin{tabular}{lrcc}
\hline Stressor category & $\underline{\mathbf{N}}$ & $\begin{array}{c}\text { Number } \\
\text { with PTSD }\end{array}$ & $\begin{array}{l}\text { Percent } \\
\text { with PTSD }\end{array}$ \\
\hline Accident at work & 1 & 1 & $100 \%$ \\
Street combat & 1 & 1 & $100 \%$ \\
Military combat & 6 & 5 & $83 \%$ \\
Killed/harmed someone & 2 & 1 & $50 \%$ \\
Incarceration & 15 & 7 & $47 \%$ \\
Witnessed violent death & 6 & 2 & $33 \%$ \\
S.U.D. & 17 & 5 & $29 \%$ \\
Motor vehicle accident & 11 & 3 & $27 \%$ \\
Assault with a weapon & 18 & 4 & $22 \%$ \\
Life-threatening illness & 6 & 1 & $17 \%$ \\
Childhood sexual abuse & 2 & 0 & $0 \%$ \\
Total & 85 & 30 & $35 \%$ \\
& & & \\
\hline
\end{tabular}

Note. S.U.D. = Sudden, unexpected death of someone close. 
Table 21

Percentage of Stressor Category Resulting in Current PTSD

\begin{tabular}{llll}
\hline Stressor category & $\underline{N}$ & Number & Percent \\
& & with PTSD & with PTSD \\
\hline Accident at work & 1 & 1 & $100 \%$ \\
Street combat & 1 & 1 & $100 \%$ \\
Killed/harmed someone & 2 & 1 & $50 \%$ \\
Military combat & 6 & 1 & $17 \%$ \\
Incarceration & 15 & 6 & $40 \%$ \\
S.U.D. & 17 & 2 & $12 \%$ \\
Assault with a weapon & 18 & 2 & $11 \%$ \\
Childhood sexual abuse & 2 & 0 & $0 \%$ \\
Motor vehicle accident & 11 & 0 & $0 \%$ \\
Witnessed violent death & 6 & 0 & $0 \%$ \\
Life-threatening illness & 6 & 0 & $0 \%$ \\
Total & 85 & 14 & $16 \%$ \\
& & & \\
\hline
\end{tabular}

Note. S.U.D. = Sudden, unexpected death of someone close. 
Table 22

Racial Distribution of Stressors for Lifetime and Current PTSD Diagnoses

\begin{tabular}{|c|c|c|c|c|c|c|}
\hline \multirow[t]{2}{*}{ Stressor category } & \multicolumn{3}{|c|}{ Lifetime PTSD } & \multicolumn{3}{|c|}{ Current PTSD } \\
\hline & $\underline{\mathbf{N}}$ & Black & White & $\underline{\mathbf{N}}$ & Black & White \\
\hline Incarceration & 7 & 2 & 5 & 6 & 2 & 4 \\
\hline Military combat & 5 & 3 & 2 & 1 & 1 & 0 \\
\hline S.U.D. & 5 & 2 & 3 & 2 & 0 & 2 \\
\hline Assaulted with weapon & 4 & 1 & 3 & 2 & 0 & 2 \\
\hline Motor vehicle accident & 3 & 2 & 1 & 0 & 0 & 0 \\
\hline Witnessed violent death & 2 & 0 & 2 & 0 & 0 & 0 \\
\hline Life-threatening illness & 1 & 0 & 1 & 0 & 0 & 0 \\
\hline Killed/harmed someone & 1 & 0 & 1 & 1 & 0 & 1 \\
\hline Accident at work & 1 & 1 & 0 & 1 & 1 & 0 \\
\hline Street combat & 1 & 1 & 0 & 1 & 1 & 0 \\
\hline Total & 30 & 12 & 18 & 14 & 5 & 9 \\
\hline
\end{tabular}

Note. $\underline{\mathbf{N}}=$ number. S.U.D. = Sudden, unexpected death of someone close. 


\section{Prevalence}

CAPS-DX diagnoses using standard scoring. Using the Clinician-Administered PTSD Scale (CAPS-DX) as the diagnostic standard and a standard scoring paradigm requiring a minimum symptom frequency of 1 (occurs once or twice a month) and a minimum intensity of 2 (mild), 30 of 100 research participants had PTSD at some point in their lives, for a lifetime prevalence of $30 \%$. Of these 30,14 currently had PTSD, for a current prevalence rate of $14 \%$. Diagnoses by security level and race are presented in Tables 23 and 24.

CAPS-DX diagnoses using the Rule of Four. Weathers and Litz (1994) stated a concern that the CAPS-DX standard scoring may over-diagnose PTSD and suggested an alternative scoring method, which was described in Chapter 3. This scoring method, the Rule of Four, suggests that a symptom only be considered as endorsed when its frequency plus intensity score totals four. This method was also used to determine the presence or absence of PTSD among the participants. Doing so, there were 26 lifetime cases, of which 13 cases were current, resulting in a lifetime prevalence rate of $26 \%$ and a current rate of $13 \%$; the number of minimum security cases dropped to 11 ; the medium security cases

dropped to 15; the number of whites with PTSD was 15; and the number of blacks with PTSD was 11. However, as will be shown, use of the Rule of Four did not affect the statistical analyses to any significant degree.

Distribution of PTSD-positive cases throughout the sample. In an effort to determine whether or not there was a pattern to the identification of PTSD-positive cases by the researcher, the Durbin-Watson statistic was computed. A D-W value of 1.97 for the independence of CAPS-DX scores confirmed that no such pattern existed and that PTSD cases are scattered randomly throughout the sample.

Comparison of CAPS-DX results with those of other diagnostic instruments. Once again, using the CAPS-DX as the diagnostic standard, the researcher examined the correspondence between the diagnosis it provided and those provided by the Keane Scale (PKS) of the Minnesota Multiphasic Personality Inventory-2 (MMPI-2) and the 
Table 23

Lifetime and Current PTSD Diagnoses by Security Level

\begin{tabular}{lrccc}
\hline Sample portion & $\underline{N}$ & $\begin{array}{c}\text { Lifetime } \\
\text { diagnosis }\end{array}$ & $\begin{array}{c}\text { Current } \\
\text { diagnosis }\end{array}$ & $\begin{array}{c}\text { Percent with } \\
\text { current DX }\end{array}$ \\
\hline Entire & 100 & 30 & 14 & $47 \%$ \\
FPC & 50 & 14 & 6 & $43 \%$ \\
FCI & 50 & 16 & 8 & $50 \%$ \\
\hline
\end{tabular}

Note. $\underline{N}=$ number. $\mathrm{DX}=$ diagnosis.

Table 24

Lifetime and Current PTSD Diagnoses by Race

\begin{tabular}{lrcccc}
\hline Sample portion & $\underline{\mathbf{N}}$ & $\begin{array}{c}\text { Lifetime } \\
\text { diagnosis }\end{array}$ & $\begin{array}{c}\text { Current } \\
\text { diagnosis }\end{array}$ & $\begin{array}{l}\text { Percent with } \\
\text { lifetime DX }\end{array}$ & $\begin{array}{l}\text { Percent with } \\
\text { current DX }\end{array}$ \\
\hline Black & 54 & 13 & 5 & $24 \%$ & $9 \%$ \\
White & 44 & 17 & 9 & $39 \%$ & $21 \%$ \\
Other & 2 & 0 & 0 & $0 \%$ & $0 \%$ \\
\hline
\end{tabular}

Note. $\underline{\mathbf{N}}=$ number. $\mathrm{DX}=$ diagnosis. 
Table 25

Mean Instrument Scores for Lifetime PTSD Diagnosis

\begin{tabular}{llll}
\hline Instrument & $\begin{array}{l}\underline{\mathrm{M}}(\underline{\mathrm{SD}}) \\
\text { with PTSD }\end{array}$ & $\begin{array}{l}\underline{\mathrm{M}(\underline{\mathrm{SD}})} \\
\text { without PTSD }\end{array}$ & $\underline{\text { V Value }}$ \\
\hline PKS & $20.5(10.5)$ & $9.2(7.2)$ & $-5.4^{*}$ \\
PDSTM & $22.3(13.6)$ & $7.4(7.8)$ & $-5.6^{*}$ \\
CAPS-DX & $72.1(19.1)$ & $20.5(15.7)$ & $-14.1^{*}$ \\
\hline${ }^{*} \mathrm{~g}<.001$ & & & \\
\hline
\end{tabular}


Posttraumatic Stress Diagnostic Scale ${ }^{\mathrm{TM}}$ (PDS ${ }^{\mathrm{TM}}$ ). As is demonstrated in Table 25, mean scores on all three instruments were significantly different for inmates with and without lifetime PTSD. In addition, the three instruments agreed completely on 80 cases. Nine of those cases were positive for current PTSD and 71 were negative. There was also agreement between the CAPS-DX and at least one other instrument on 96 of 100 cases and on all 14 current PTSD cases.

For the PKS, a cutoff of 24 suggested by Herman et al. (1996) was used to indicate the possible presence of PTSD. PKS scores of 24 or more and CAPS-DX diagnoses were in agreement for 90 cases, 81 without a current diagnosis and 9 with one. Of the 10 cases where there was disagreement, five of the PKS scores were false positives and five were false negatives. This resulted in a sensitivity of .64 and a specificity of .94 for the PKS. Of the participants registering false positive scores, four had non-current, lifetime PTSD according to the CAPS-DX, while only one had no lifetime diagnosis. Although lowering the PKS cutoff to 22 increased the identification of current PTSD cases by $11 \%$, it also increased false positives by $40 \%$. Increasing the cutoff to 25 or 26 resulted in the same number of current PTSD cases, and six and five false positives, respectively. Therefore, cutoff of 24 seems appropriate for this sample.

The PDSTM and the CAPS-DX were in agreement on 86 cases, 72 without current PTSD and 14 with it. All of the 14 cases where the instruments disagreed were false positives. This resulted in a sensitivity of 1.0 and a specificity of .84 . Of the 14 false positives, eight had a non-current, lifetime PTSD diagnosis per the CAPS-DX and six had no lifetime diagnosis.

\section{PTSD Symptoms}

Mean number of symptoms experienced. To satisfy the diagnostic criteria for PTSD, a person must not only satisfy Criterion $A$ but also endorse a minimum of one reexperiencing symptom, three avoidance symptoms, and two arousal symptoms. In this study, participants with lifetime PTSD endorsed a mean of $3.5(\underline{S D}=1.1)$ reexperiencing symptoms, $4.9(\underline{S D}=1.3)$ avoidance symptoms, and $3.8(\underline{S D}=0.8)$ arousal symptoms on the CAPS-DX. Participants with a lifetime PTSD diagnosis endorsed significantly more re-experiencing symptoms, $\underline{\mathrm{t}}(98)=-11.2, \mathrm{p}<.001 ;$ avoidance symptoms, $\underline{\mathrm{t}}(98)=-12.3, \mathrm{p}$ 
$<.001$; and arousal symptoms, $\underline{t}(87)=-11.1, \mathrm{p}<.001$, than participants without lifetime PTSD. These findings were not altered when race and security level were considered.

CAPS-DX symptom scores. Each of the 17 CAPS-DX symptoms is given a score ranging from 0-8. The total of these individual symptom scores provides a continuous score which can range from 0-136. To be diagnosed with PTSD, a person must first satisfy Criterion $\mathrm{A}$, have a distribution of symptoms as discussed in the preceding paragraph, and have a continuous CAPS-DX score of at least 18. Therefore, it is possible for an individual to have a continuous score of 18 or more and not be diagnosed with PTSD because either Criterion $A$ was not met, the required number of symptoms in each category was not met, or both. In this sample, the mean CAPS-DX continuous score for men without lifetime PTSD was 20.5 (SD = 15.7), and mean continuous scores for the reexperiencing, avoidance, and arousal clusters were $6.1(\underline{S D}=5.9), 6.5(\underline{S D}=8.2)$, and $8.0(\underline{S D}=7.5$ ), respectively. By contrast, the mean CAPS-DX continuous score for men with lifetime PTSD was $72.1(\underline{S D}=19.1)$, with mean continuous scores for the reexperiencing, avoidance, and arousal clusters of $19.8(\underline{S D}=7.7), 29.1(\underline{S D}=10.2)$, and $23.2(\underline{S D}=6.1$ ), respectively. The mean scores for participants with and without lifetime PTSD were compared using t-tests. The mean scores for re-experiencing, $t(98)=-9.71, \mathrm{p}$ $<.001$; avoidance, $\mathrm{t}(46)=-10.72, \mathrm{p}<.001 ;$ and arousal, $\mathrm{t}(98)=-9.78, \mathrm{p}<.001$, were all higher for the lifetime PTSD group than the group without lifetime PTSD. These findings were not altered when race and security level were considered.

\section{Multiple Linear Regression Analysis}

The choice of a target variable. A multiple linear regression analysis can be used either to predict or explain the magnitude of a dichotomous or continuous variable. For this analysis, the researcher has chosen to use the CAPS-DX score (Capscore), which is a continuous variable, as the target variable. This choice was made because the variable is indicative of PTSD symptomatology even for participants who have not been diagnosed with lifetime PTSD. As has been noted, a minimum score of 18 on the CAPS-DX is required for a PTSD diagnosis, along with satisfaction of Criterion $\mathrm{A}$, and a symptom endorsement distribution of at least one reexperiencing symptom, three avoidance symptoms, and two arousal symptoms. Therefore, it is possible for a participant to have a 
score of 18 or more on the CAPS-DX and not have PTSD. In fact, the average CAPS-DX continuous score for sample participants without PTSD is $20.5(\underline{S D}=15.7)$. This indicates that many of the participants in this sample who did not satisfy Criterion $A$, or did not meet the symptom distribution requirements, or both, still exhibited PTSD symptomatology. By using the CAPS-DX continuous score as the regression target variable, it will be possible to make statements about inmates with and without PTSD.

Selection of independent variables. As has been noted, PTSD predictor variables are not consistent across populations. Therefore, a large number of independent variables were selected from information provided by the research questionnaire (see Appendix II) and the assessment instruments to find out which ones would enter the regression equation. The following variables were chosen: The participant's age; birth category ( $1=$ first child, 2 = middle child, and $3=$ last child); combat experience $(1=$ yes and $0=$ no); education level $(1=$ ninth grade or less, $2=$ more than nine, but less than 12 years, $3=$ high school graduate, 4 = some college, $5=$ college graduate, $6=$ graduate school); IQ; marital status (single, married, separated, divorced, widowed); native language ( $1=$ English and $0=$ other); number of children; number of marriages; number of CAPS-DX potentially traumatic events experienced; number of siblings; previous treatment for a mental disorder $(1=$ yes and $0=n o)$; convicted of a violent crime $(1=$ yes and $0=n o)$; previous problems with alcohol and/or drugs $(1=$ yes and $0=$ no); race; active religious participation ( $1=$ yes and $0=$ no); security level; and the childhood SES continuous score $(0-66)$. Means and standard deviations for these variables, where appropriate, are presented in Table 26, and correlations are presented in Table 27.

Regression results. All of the variables listed above were entered into a forward regression model, maintaining a participant to variable ration of 5:1. Three of the variables entered the regression equation, the number of potentially traumatic events experienced $(\mathrm{t}=3.8, \mathrm{p}<.001)$; previous treatment for a mental disorder $(\mathrm{t}=2.9, \mathrm{p}<.01)$; and number of siblings $(\underline{t}=-2.0, \underline{\mathrm{p}}<.05)$. This resulted in a multiple $\underline{\mathrm{R}}=.57$, an $\underline{\mathrm{R}}$ squared $=.33$, and an adjusted $\underline{\mathrm{R}}$ squared $=.30$. The number of potentially traumatic events experienced explained $23 \%$ of the variance in CAPS-DX continuous score, a prior 
Table 26

Means and Standard Deviations for Regression Variables

\begin{tabular}{lll}
\hline Variable & Mean & Standard deviation \\
\hline Age & 37.9 & 10.4 \\
Birth category & 1.9 & 0.7 \\
Combat experience & 0.1 & 0.3 \\
Childhood SES score & 34.4 & 14.0 \\
Education level & 3.2 & 1.5 \\
IQ & 95.4 & 12.9 \\
Mental TX & 0.2 & 0.3 \\
Native language & 0.9 & 0.3 \\
Number of children & 2.3 & 1.8 \\
Number of marriages & 0.9 & 0.9 \\
Number of siblings & 4.1 & 3.4 \\
Traumatic events & 8.7 & 5.6 \\
Violent crime & 0.4 & 0.5 \\
\hline
\end{tabular}

Note. Mental TX = Previous treatment for a mental disorder. Traumatic events $=$ The number of potentially traumatic events endorsed on the CAPS-DX. Violent crime $=$ convicted of a violent crime. 
Table 27

Bivariate Correlations

Variable pairing Correlation Significance

CAPS-DX score and age

$-.03$

.77

CAPS-DX score and alcohol/drugs

.23

.02

CAPS-DX score and birth category

$\begin{array}{ll}.14 & .17\end{array}$

CAPS-DX score and CAPS-DX events

$.45 \quad .00$

CAPS-DX score and combat experience

.16

.12

CAPS-DX score and education level

.05

CAPS-DX score and IQ

.06

CAPS-DX score and marital status

.20

CAPS-DX score and mental treatment

.38

CAPS-DX score and native language

.07

CAPS-DX score and number of children

$-.02$

CAPS-DX score and number of marriages

.12

CAPS-DX score and number of siblings

$-.14$

CAPS-DX score and race

.03

CAPS-DX score and religious activity

.05

CAPS-DX score and security level

.04

CAPS-DX score and SES

$-.02$

CAPS-DX score and violence 
Table 28

Multiple Linear Regression Results

\begin{tabular}{lrrrrr} 
Variable & B & SE B & Beta & t & R squared \\
\hline Capsevent & 1.85 & .49 & .38 & $3.8^{* *}$ & .23 \\
Mental TX & 28.64 & 9.86 & .29 & $2.9^{* *}$ & .29 \\
NoSibs & -1.56 & .77 & -.19 & $-2.0^{*}$ & .33 \\
(Constant) & 22.27 & 6.01 & & $3.7^{* * *}$ & \\
\hline
\end{tabular}

Note. Capsevent $=$ The number of potentially traumatic events experienced.

Mental TX = Prior treatment for a mental disorder.

NoSibs $=$ The number of siblings in the childhood family.

${ }^{*} \mathrm{p}<.05 .{ }^{*} \mathrm{p}<.01 . * * * \mathrm{p}<.001$. 
diagnosis explained an additional $6 \%$, and the number of siblings explained the last $4 \%$. The regression equation is presented in Table 28.

Examination of regression assumptions. Studentized deleted residuals (SDR's) were then used to check the assumptions required for a regression analysis. These assumption include the independence of observations, homogeneity of variance, distribution normality, and a linear relationship between dependent and independent variables (Norusis, 1995). Examination of a scatterplot of the SDR's and the predicted value of the CAPS-DX continuous score indicated that neither the constant variance nor the linearity assumption had been violated. A scatterplot of the SDR's and order of scores indicated that scores were independent of one another, as did the value of the DurbinWatson test (1.97). In addition, a stem and leaf plot of the SDR's indicated a normal distribution and the $\mathrm{K}-\mathrm{S}$ Lilliefors statistic confirmed this $(\underline{K}-\mathrm{S}=.07, \mathrm{p}>.20)$. Therefore, the assumptions for a multiple linear regression analysis have been met.

Analysis of the Study's Hypotheses

Hypothesis 1

That the current and lifetime rates of PTSD among male inmates will be higher than those exhibited in the general population and higher than those found in previous estimates involving American inmates. Lifetime prevalence rates of PTSD for males in the general population have ranged from 0.5 - 6\% (Breslau, Davis, Andreski, \& Peterson, 1991; Davidson et al., 1991; Helzer, Robins, \& McEvoy, 1987; and Kessler et al., 1995). In an effort to be as conservative as possible, the $6 \%$ figure was compared with the lifetime rate of $30 \%$ found in this study. A chi-square analysis using raw scores and comparing the identified lifetime diagnoses for inmates (30 PTSD-positive, 70 PTSDnegative) against the most conservative general population rate for males (six PTSDpositive, 94 PTSD-negative) indicated that a significant difference existed, $\chi 2(1, \underline{N}=$ $100)=102.1, \mathfrak{p}<.0001$. As the previous lifetime prevalence rate for males in American prisons was also six in 100 (Hodgins \& Cote, 1990), the difference between that number and the finding of the current study is also significant at the $\mathrm{p}<.0001$ level. When the identified number of PTSD-positive and negative cases were compared with the number 
found in a Canadian penitentiary (13 PTSD-positive, 87 PTSD-negative) (Hodgins \& Cote), the chi-square result remained significant, $\chi 2(1, \underline{N}=100)=25.6, \mathrm{p}<.0001$.

Unfortunately, the issue of whether or not the finding of $14 \%$ current PTSD rate is significantly greater than findings for the general population or other prison populations is more difficult, if not impossible, to determine. The researcher was not able to identify any current rates of PTSD for the general population and could only find one six-month rate for a prison population. That rate was 3.9\%, identified by Hodgins and Cote (1990) in their study of Canadian inmates. However, the $3.9 \%$ rate may be low. Hodgins and Cote provided two kinds of lifetime prevalence figures for PTSD, one for which PTSD was the sole diagnosis for an inmate (5.9\%) and one for which it was one of many diagnoses (13\%). As the six-month rate of $3.9 \%$ refers to only those inmates who had no other diagnosis but PTSD, we are left not knowing what the six-month rate is for inmates with multiple diagnoses. One way of estimating the six month rate for multiple diagnoses would be to multiply $3.9 \%$ by 2.2 , which is the factor by which the lifetime rate for multiple diagnoses (13\%) is greater than the lifetime rate for a sole diagnosis (5.9\%). Doing this suggests a six-month prevalence rate of $8.58 \%$. A chi-square analysis comparing this figure with the $14 \%$ found in the current study was significant, $\chi 2(1, \underline{N}=$ $100)=4.204, \mathrm{~g}=.0403$. Therefore, the first hypothesis was supported.

Hypothesis 2

That inmates incarcerated in a medium security facility will manifest a higher prevalence of PTSD than those in a minimum security facility. Here again, a chi-square analysis using raw scores was employed to determine whether the 14 PTSD-positive cases among minimum security inmates differed significantly from the 16 PTSD-positive cases found among medium security inmates. The result was not significant. Even when the Rule of Four was utilized and number of PTSD-positive minimum security inmates was reduced to 11, the result was still not significant. Therefore, the second hypothesis was not supported.

\section{Hypothesis 3}

That the majority of inmates who are identified as PTSD-positive will not have been previously identified as such by corrections officials. Of the 30 participants who 
were identified with lifetime PTSD, 25 permitted the researcher to view their confidential psychology files to determine whether of not corrections officials were aware of the problem. Only one inmate had been identified as having had PTSD and this had been diagnosed by the Veterans Administration. While none of the 14 men with current PTSD had been identified as such, five had been diagnosed with another problem such as anxiety, depression, or bipolar disorder. Therefore, the third hypothesis was supported.

\section{Hypothesis 4}

That PTSD-positive inmates will have lower full scale intelligence quotient (IO) estimates than inmates without PTSD. A t-test was used to examine the validity of this hypothesis. When mean IQ scores for participants with PTSD $(\underline{N}=30, \underline{M}=95.6, \underline{S D}=$ 11.9)) were compared with mean IQ scores for participants without PTSD $(\underline{N}=70, \underline{M}=$ $95.3, \underline{S D}=13.5$ ), the result was not significant. Therefore, the fourth hypothesis was not supported.

\section{Hypothesis 5}

That the childhood socioeconomic status of PTSD-positive inmates will be lower than that of inmates without PTSD. To examine this hypothesis, continuous childhood SES mean scores were computed for both groups and then compared using a t-test. The mean for the PTSD-positive group $(\underline{\mathrm{N}}=30)$ was $33.1(\underline{\mathrm{SD}}=13.5)$ and the mean for the PTSD-negative group $(\underline{N}=70)$ was $35.0(\underline{S D}=14.3)$. The results of the test were not significant, and the fifth hypothesis was not supported.

\section{Hypothesis 6}

That African-American and Hispanic inmates will have higher PTSD prevalence rates than Caucasian inmates. This study identified PTSD lifetime prevalence rates of $14 \%$ for Hispanics (one of seven Hispanic inmates was PTSD-positive), 26\% for AfricanAmericans (13 of 50 African-American inmates were PTSD-positive), and 39\% for Caucasians ( 16 of 41 Caucasians were PTSD-positive). As Caucasians had the highest rate of PTSD, the fourth hypothesis was not supported. However, it is more common in the BOP to classify inmates as black or white, with Hispanics falling into either group. When inmates are classified in this manner, the rate of lifetime PTSD among blacks becomes $24 \%$, while the rate among whites is $39 \%$. A chi-square analysis comparing 
these prevalence rates was significant, $\chi 2(1, \underline{\mathrm{N}}=98)=3.986, \mathrm{p}<.05$. Therefore, even though the sixth hypothesis was not supported, its inverse was, and the study identified a significant difference in lifetime PTSD prevalence between blacks and whites.

\section{Hypothesis 7}

That, for some inmates, incarceration itself will have been a traumatic stressor. Fifteen inmates identified incarceration as their most traumatic stressor and seven of those had lifetime PTSD. Therefore, the seventh hypothesis is supported. However, it should be noted that when these seven cases were examined closely, it became apparent that incarceration was the actual precipitating stressor in only one case and a possible precipitating stressor in one other. While the hypothesis remains supported, more will need to be said about it in the discussion section.

\section{Summary of Hypothesis Support}

In summary, the study's first, third, and seventh hypotheses were supported, while its second, fourth, fifth, and sixth were not. 


\section{Chapter 5: DISCUSSION}

\section{Introduction}

This study's hypotheses are divided into two groups: Primary and secondary. The primary group of hypotheses states that incarcerated men are more likely to have postraumatic stress disorder (PTSD) than men in the general population, that previous estimates of PTSD among male inmates are low, that PTSD prevalence rates differ by security level, and that prison authorities are unaware of the number of men under their control who have PTSD. The secondary group focuses attention on specific aspects of the background of men with and without PTSD. These hypotheses state that lower IQ, lower familial socioeconomic status (SES), and minority racial status are characteristic of inmates with PTSD, and that incarceration itself can be a precipitant of the disorder.

These hypotheses are important because the presence of PTSD often suggests the presence of other problems such as anger, antisocial behavior, depression, and substance abuse, all of which can pose a threat to the security and welfare of all inmates, as well as the institution. In addition, the presence of PTSD may make it more difficult for inmates to engage in educational programs, such as drug and alcohol education and the attainment of high school equivalency certificates (GED's).

However, without basic prevalence information, it is difficult, if not impossible, to make arguments for addressing the problem. Identification of a problem and its extent are steps that must precede remediation. Once corrections officials are aware that inmates in general, as well as specific groups of inmates, exhibit higher rates of PTSD, they can devise programs and services to address the behavioral and emotional sequelae of this disorder. In addition, once aware of the problem, corrections officials can screen drug and alcohol and GED program applicants for PTSD so that the unaddressed aspects of the disorder will not hinder successful participation in these important activities.

\section{Study Findings}

\section{Primary Hypotheses}

Lifetime and current prevalence. The assessment of 100 male inmates for PTSD identified 30 with lifetime PTSD, of whom 14 had the disorder at the time of the study ("current" PTSD). Both the lifetime (30\%) and current (14\%) prevalence rates were 
significantly higher than rates previously established for men in the general population and for male inmates. This is the main finding of this study.

One question that arises is how these prevalence rates compare with those found for other high-risk groups. The lifetime and current prevalence rates for inmates identified in this study are displayed in Table 29 along with PTSD prevalence rates identified for other groups of individuals, such as combat veterans, police, firefighters, survivors of motor-vehicle accidents, survivors of natural disasters, assault victims, and homeless men. Among the groups listed above, the range for lifetime PTSD prevalence is $15-46 \%$, while the range for current PTSD is $15-16 \%$. These figures place inmates in the company of groups that are considered highly likely to have or have had PTSD as a result of their life experiences. Therefore, as a group, inmates need to be viewed as people who are at risk of having or having had PTSD because of who they are and, as will be shown, of what they have experienced.

Prevalence by security level. The results of this study suggest that the PTSD prevalence rate is the same for men in the minimum and medium security facilities. This means that security level is not an indicator of the presence of PTSD and that men with the disorder will just as likely be found among inmates who have committed less serious offenses as among inmates who have committed more serious offenses. This finding stands in contradiction to the study's second main hypothesis.

The hypothesis, that inmates in medium security manifest higher rates of PTSD than inmates in minimum security, was based on the assumption that security level and the number of potentially traumatic lifetime events an inmate had experienced were related. However, it turns out there is no statistical difference between the average number of potentially stressful events experienced by men in the minimum and medium security facilities. Men in the former experienced an average of $8.1(\underline{S D}=5.5)$ potentially traumatic events, while men in the latter experienced an average of $9.3(\underline{S D}=5.7)$ potentially stressful events.

Even though this hypothesis was not supported, it would be of value to know if the assumption upon which it was based was supported. If the thinking underlying the original hypothesis is correct, then the inmate sample as a whole should have experienced 
Table 29

\section{Lifetime Prevalence Rates by Group}

Trauma group

Lifetime PTSD

Current PTSD

Federal inmates (1)

$30 \%$

$14 \%$

Motor vehicle accident victims (2)

$46 \%$

Aggravated assault victims (3)

$37 \%$

Firefighters (4)

$16 \%$

Police (5)

$26 \%$

Homeless men (6)

$18 \%$

Myocardial infarction survivors (7)

$15 \%$

Combat veterans (8)

$31 \%$

$15 \%$

Disaster victims (9)

$45 \%$

(1) Current study, (2) Blanchard et al. (1994), (3) Kilpatrick et al. (1987), (4) McFarlane (1988), (5) Martin et al. (1986), (6) North \& Smith (1992), (7) Kutz et al. (1997),

(8) Kulka et al. (1990), and (9) Green et al. (1990). 
a greater number of traumatic events than men who are not incarcerated. In addition, those inmates who have experienced more potentially traumatic events should demonstrate increased PTSD symptomatology.

Overall, participants in the current study experienced an average of $8.7(\underline{S D}=5.6)$ potentially traumatic events during their lifetime. Inmates with PTSD experienced an average of $12.4(\underline{S D}=6.9)$ potentially traumatic stressors, while inmates without PTSD experienced an average of $7.1(\underline{S D}=4.1)$. As was reported in Chapter 4 , the difference between the number of events experienced by those with and without lifetime PTSD is significant.

Vrana and Lauterbach (1994) assessed 234 male college students for potentially traumatic experiences and found that the mean for their group was 3.0 events. By contrast, inmates, on average, experienced nearly three times as many potentially traumatic events as Vrana and Lauterbach's male participants. In addition, PTSD-positive inmates experienced four times as many potentially traumatic events as the sample of college men. Vrana and Lauterbach also found that psychopathology increased with the number of potentially traumatic events experienced. And Thoits (1983) pointed out that there is a positive correlation between psychological stress and the number of unpleasant events experienced.

When CAPS-DX continuous scores, which are indicative of each participant's level of PTSD symptomatology, were regressed on a variety of independent variables, the number of potentially traumatic events experienced explained $22.5 \%$ of the variance associated with the dependent variable, $\underline{\mathrm{F}}(1,77)=22.4, \mathrm{p}<.001$. Thus, a participant's PTSD symptomatology tended to increase as the number of traumatic events he had experienced increased.

These findings suggest that the basic reasoning underlying the hypothesis that PTSD prevalence increases with security level is sound. But if that's true, why was the number of stressors experienced by men at different security levels the same? The only key variable that might help account for the similarity is SES. Other key variables -- the racial distribution of the minimum and medium security inmate groups, their average age, and their average IQ -- all differ significantly by security level. Therefore, these variables 
cannot possibly account for a similar number of events experienced. But a common childhood SES could account for the similarity, in that it may have helped equalize the number of traumatic events experienced by these groups. It stands to reason that men from a similar childhood SES will have a greater likelihood of experiencing similar lifetime events. Given the variables this study examined, childhood SES seems the most likely candidate for explaining the similar number of potential stressors experienced by men in minimum and medium security levels.

Awareness of corrections officials of the presence of PTSD. The study's third primary hypothesis stated that corrections officials are not aware of the majority of PTSD-positive cases in their institution. This hypothesis was based on research by Teplin (1990a) suggesting that mental disorders often go unrecognized in correctional settings unless they are already part of an inmate's record. In this study, none of the 14 inmates with current PTSD were identified as such by corrections psychologists. However, five were diagnosed with another psychological problem, including an unspecified anxiety disorder, depressive symptoms, and bipolar disorder. Therefore, corrections psychologists at the research site noted psychological problems in $36 \%$ of the currently PTSD-positive population. It is commendable that over a third of these men were identified as currently experiencing psychological difficulties; but it is disappointing, though not surprising, that none of them were diagnosed with PTSD. Therefore, this study supports Teplin's position that mental disorders often go undiagnosed in a correctional setting.

There may be costs associated with this failure to diagnose. If $14 \%$ of all inmates have current PTSD, then, on average, at least $14 \%$ of inmates involved in drug and alcohol and GED programs will have current PTSD. The presence of this disorder may make it more difficult for these men to succeed at either program, and their failure to succeed may contribute to their recidivism. It would also be interesting to know the rate of current PTSD for men held in solitary confinement in the BOP's Special Housing Units (SHU) for behavioral problems or for men on suicide watch. If current prevalence rates are shown to be elevated among these groups, then addressing their PTSD symptoms might be an effective way to enhance inmate and staff safety. This is a potential future research question. 


\section{Secondary Hypotheses}

IQ and SES differences for inmates with and without PTSD. Hypothesis four stated that PTSD-positive inmates have lower full-scale IQ scores than inmates without PTSD. However, mean IQ scores for participants with and without PTSD were 95.6 (SD $=11.9)$ and $95.3(\underline{S D}=13.5)$, respectively. As these scores were not statistically different from one another, this hypothesis was not supported. Along the same lines, hypothesis five states that PTSD-positive inmates will be from a lower socioeconomic background than inmates without PTSD. But the mean SES score for inmates with PTSD was 33.1 $(\underline{S D}=13.5)$, while the mean score for inmates without PTSD was $35.0(\underline{S D}=14.3)$, and this difference is not significant.

These results leave us to wonder why IQ and SES are not predisposing factors for an inmate population, when they are clear predisposing factors for a combat population (Kulka et al., 1990). In both cases, the answer may simply be that the sample is too small or too homogeneous to allow the detection of IQ and SES differences. Or it may be that the mean number of stressors experienced by inmates is so high as to overcome any prophylactic benefits provided by higher IQ and higher SES.

In a sense, this latter suggestion is supported by the fact that the lifetime prevalence rate for this male population (30\%) is close to the lifetime prevalence rate identified for incarcerated women (33\%) by Teplin, Abram, and McClelland (1996). As has been shown, the lifetime PTSD rate for males in the general population is approximately half that of females (Breslau et al., 1991, Kessler et al., 1995). This occurs despite the fact that males usually experience more potentially traumatic stressors than females (Vrana \& Lauterbach, 1994). Therefore, the findings of Teplin et al. may indicate that the prevalence differential vanishes as men are exposed to an ever greater number of potentially traumatic stressors. It would be interesting to know if there is a difference in the average number of traumatic events experienced by male and female inmates, and if there is a point where IQ and SES no longer play some sort of role in preventing PTSD.

Despite the fact that IQ and SES are not different for inmates with and without PTSD, two other variables emerge as possibly predisposing an inmate for a later diagnosis of PTSD. The first is past treatment for a mental disorder. The variable entered 
the regression equation for the prediction of CAPS-DX continuous scores and accounted for $6.9 \%$ of the variance. In addition, the number of siblings in an inmate's family also entered the regression equation and accounted for $3.6 \%$ of the variance.

In both cases, it seems reasonable that these variables entered the regression equation. As concerns the number of siblings, more brothers and sisters may provide increased emotional support in time of crisis (a kind of ready-made support network). Or, living in a large family may provide a person with enhanced abilities to interact with and seek support from others. Certainly, having been previously diagnosed with a mental problem may be indicative of an individual's tendency to decompensate under stress. In fact, Helzer, Robins, and McEvoy (1987) also found a past history of psychological problems to be a predisposing factor for subsequent development of PTSD. Whatever the reasons, in this sample more siblings and a lack of a previous mental diagnoses are indicative of less severe PTSD symptomatology.

Racial differences for inmates with and without PTSD. Hypothesis six states that minority (black and Hispanic) inmates have a higher PTSD prevalence rate than nonminority (white) inmates. While this hypothesis was not supported, its inverse was. The lifetime rate of PTSD for whites is $41 \%$, while it is $22 \%$ for minorities. This difference is significant.

It is unclear if these results diverge from what others have found. For example, rates of lifetime PTSD identified by Kulka et al. (1990) were higher among minority group Vietnam combatants than among non-minority combatants. However, when the researchers controlled for combat exposure, there was no difference by race in PTSD rates. Why then was a significant inverse difference identified for inmates? Can it be that blacks have become inured to traumatic experience, but whites have not? Some blacks in the sample stated that trauma was "just a way of life" for them, "something you come to expect." One man even talked about a game blacks play called "Playing the Dozens," which he said had been around since slavery times. In it, blacks hurl insults at one another about such things as their mothers, physical attributes, and intelligence. The purpose of the game is to get blacks used to insults so that they can cope with them in the real world. 
To the best of my knowledge, there is no comparable game in the white culture. Therefore, inurement seems a possibility.

As we have seen, the number of potentially traumatic stressors experienced by participants in this study is a strong indicator of PTSD symptomatology. Can it be that whites experience more traumatic stressors than blacks? The answer is no. For the entire sample, whites experienced a mean of $8.9(\underline{S D}=6.2)$ potentially traumatic stressors, while blacks experienced a mean of $8.8(\underline{S D}=5.1)$. In addition, the mean number of potentially traumatic events experienced by blacks and whites who were PTSD-positive or PTSD-negative did not differ significantly.

Does this mean that while the number of stressful events experienced is a predictor of PTSD for the sample as a whole, it is not a predictor for blacks in particular? When the regression analysis was re-run for a split-file of blacks and whites, the number of potentially traumatic events experienced was still the most important predictor of CAPS-DX continuous scores, accounting for $18.3 \%$ of the variance for blacks, $\underline{F}(1,36)=$ $8.1, \mathrm{p}=.007)$, and $23.1 \%$ of the variance for whites, $\mathrm{E}(1,37)=11.1, \mathrm{p}=.002$.

Does the difference in prevalence rates mean that whites are over-reporting their symptoms and blacks are under-reporting them? Of the possibilities suggested so far, this may be the most plausible explanation. This study's results may have been influenced by the race and age of the investigator and the racial distribution of the population from which the sample was drawn. The investigator is a white male, who at the time the research was conducted was 51 years old. Sue, Zane, and Young (1994) have pointed out that a whole array of issues in addition to race may influence the relationship between black clients and therapists, including social class, attractiveness, similarity of attitudes, and age. Therefore, it may be that the investigator was just too different from the black participants to establish the kind of rapport necessary for them to disclose their PTSD symptoms. For example, in addition to the racial difference, there was an average age difference of 15 years between the investigator and black participants and an average educational difference of 10 years. For whites, the average age difference was 10 years and the average educational difference was also 10 years. These personal differences may have been more important for blacks than for whites. 
As Halpern (1993) has pointed out, being a member of a minority group increases one's risk for mental illness. But Halpern is not referring to global minority status; he is referring to local minority status. In other words, the fact that whites are in the minority at the BOP site in Cumberland may be a reason for exaggerated symptomatology, while the fact that blacks are in the majority may be a reason for understated symptomatology. In his work in the group therapy field, Davis (1979) found that blacks tend to feel comfortable in groups when the ratio is $50: 50$ or better, black to white, whereas whites only feel comfortable in a group when the ratio is $70: 30$ or better, in their favor. What all this may suggest is that the racial environment also plays a role in PTSD symptomatology.

The current study provides statistical support for this view. At this research site, blacks comprised approximately $65 \%$ of the total population and whites made up approximately $35 \%$. Although there is no significant difference in the numbers of blacks and whites in minimum security portion of the sample, there is a significant difference in the number of blacks and whites in medium security portion of the sample, $\chi 2(48)=$ $6.75, \mathrm{p}<.01$.

If Halpern is correct, then whites should evidence about the same amount of PTSD symptomatology as blacks in minimum security, but greater PTSD symptomatology than blacks in medium security. To find out if this was the case, a $2 \times 2$ analysis of variance (ANOVA) was conducted, using CAPS-DX scores as the dependent variable and race and security levels as the independent variables. The results of the ANOVA indicated there was a significant interaction between race and security level, $\mathrm{F}(1,94)=7.47, \mathrm{~g}<.01$. The mean CAPS-DX scores for blacks and whites in minimum security were $38.7(\underline{S D}=25.0)$ and $32.1(\underline{S D}=30.2)$, respectively. And the mean CAPSDX scores for blacks and whites in medium security were $30.6(\underline{S D}=24.0)$ and $56.2(\underline{S D}$ $=34.5$ ), respectively. When t-tests were run on the CAPS-DX means for blacks and whites, the one for minimum security was not significant and the one for medium security was, $\underline{t}(46)=-2.98, \underline{p}<.01$. These results support Halpern's suggestion that the diagnosis of symptoms of mental illness is influenced by local minority status. 
Therefore, of the suggestions put forth to explain the results obtained for the sixth hypothesis, local minority status, possibly resulting in under- and over-reporting of symptoms, appears to be the most powerful. However, a need to become inured to distressing events may have also contributed to under-reporting of symptoms for blacks. An improved research design involving a greater number of participants, sites with different racial groups in the minority and majority, and investigators who are more similar to the participants in terms of age and race would help explore these possibilities further.

Incarceration and other events as PTSD stressors. The study's final hypothesis states that incarceration itself is a traumatic stressor, satisfying Criterion A of the DSMIV's (1994) PTSD diagnostic criteria. As 15 inmates identified incarceration as their most traumatic stressor, of whom seven have lifetime PTSD, the hypothesis is supported. However, incarceration is often mixed up with other stressful events, and there is only one case in which it is the clear precipitant of PTSD symptoms. Although this suggests that incarceration can be reasonably included among the events that may precipitate PTSD, it also makes sense to examine whether or not PTSD in this sample is the result of discrete or multiple stressors.

Of the study's 100 participants, 15 had not experienced a traumatic stressor that satisfied Criterion A. Of the 85 men who identified a traumatic stressor that satisfied Criterion $\mathrm{A}$, those most frequently cited are assault with a weapon (18), the sudden, unexpected death of someone close (17), incarceration (15), and motor vehicle accidents (11). Together, these four stressors are involved in $72 \%$ of all cases in which Criterion A was satisfied; $63 \%$ of all lifetime PTSD diagnoses; and $71 \%$ of all current PTSD diagnoses. Of these four, it appears that incarceration resulted in a lifetime PTSD diagnosis $47 \%$ of the time and a current diagnosis $40 \%$ of the time; that a sudden, unexpected death resulted in a lifetime diagnosis $29 \%$ of the time and a current diagnosis $12 \%$ of the time; that motor vehicle accidents resulted in a lifetime diagnosis $27 \%$ of the time and a current diagnosis none of the time; and that assault with a weapon resulted in a lifetime diagnosis $22 \%$ of the time and a current diagnosis $11 \%$ of the time. What is needed is a closer look at these top four stressors to determine whether or not they are the 
sole precipitants of an inmate's PTSD. As it is the focus of the study's last hypothesis, we'll begin with incarceration.

In their validation study for the Posttraumatic Stress Diagnostic Scale ${ }^{\text {TM }}$ (PDSTM), Foa, Cashman, Jaycox, and Perry (1997) found that 14\% of 248 participants identified incarceration as a traumatic event. However, none of these individuals was incarcerated at the time of the study, and none identified incarceration as the most traumatic event he or she had experienced. In the current study, use of the PDS ${ }^{\mathrm{TM}}$ identified 14 men for whom incarceration fulfilled the requirements of Criterion $\mathrm{A}$, and seven of those 14 were diagnosed by the PDS ${ }^{\mathrm{TM}}$ as having current PTSD. By comparison, in responding to the CAPS-DX, 15 inmates identified incarceration as their most traumatic stressor, and of those 15, six were diagnosed as having current PTSD.

Different response patterns in the PDS ${ }^{\mathrm{TM}}$ validation study and the current study may suggest that the more recent a traumatic event, the more likely it is to be associated with symptoms of PTSD. To determine whether or not this hypothesis makes sense, it is helpful to review the trauma histories of the seven men who identified incarceration as the precipitant of their current or past PTSD. Of these seven, incarceration was the clear precipitant for only one. At the time of the study, he was a 50-year-old white accountant, and prior to his arrest, conviction, and incarceration, the only other traumatic events he experienced were the death of his father to heart disease and his mother's vascular dementia. One other man, a 27-year-old African-American, could only identify incarceration and the sudden, unexpected death of his mother-in-law as having been traumatic. However, he had experienced, witnessed, or learned about a total of 16 potentially traumatic events, including having experienced childhood sexual abuse. Therefore, while it is possible that this second man's PTSD is the result of incarceration, it is less likely than in the first case.

All of the other five men who identified incarceration as the precipitant of their PTSD have very complicated trauma histories. One man was exposed to 11 potentially traumatic stressors, including seeing his father murdered and nearly dying when his own carotid artery was severed by a knife. Despite the fact that he identified both of these as satisfying Criterion $\mathrm{A}$, he cited the witnessing of assaults in state prison as his most 
traumatic event. A former policeman who identified incarceration as his most traumatic event experienced 14 potentially traumatic stressors, including being repeatedly assaulted and the viewing of multiple violent deaths. As the lifetime rate of PTSD among police officers is $26 \%$, it is very possible this man had PTSD before coming to prison. Another man, who experienced a total of 21 potential stressors, had shot another youth in a gang fight at 19 , had been shot numerous times, and had nearly died when his girlfriend stabbed him three times in his sleep. One inmate who said that seeing men stabbed in state prison had precipitated his PTSD symptoms, had been physically abused for 15 years by his older brother. It was involvement in criminal activity with this same brother that resulted in his incarceration. Finally, one inmate's incarceration was linked to his abuse of a girlfriend who subsequently turned him in to police for a bank robbery. That abuse and subsequent punishment may well have become linked with the physical abuse of his first wife, her divorce from him, and his subsequent depression and multiple suicide attempts. Despite all this linkage, the man believed incarceration was responsible for his PTSD symptoms.

Therefore, of the seven men who identified incarceration as the stressor that had precipitated their PTSD symptomatology, there was an unambiguous relationship between incarceration and PTSD for only one inmate. This supports the idea that some inmates identify incarceration as the cause of their PTSD symptoms simply because it is the most recent in a string of many traumatic experiences. It appears the Law of Recency is alive and well.

Now let's look at the study's other most common stressors: Assault with a weapon; the sudden, unexpected death of someone close; and motor vehicle accidents. In and of themselves, these stressors can and do precipitate PTSD. Breslau, Kessler, Chilcoat, Schultz, Davis, and Andreski (1998) reported that in a general population sample, having experienced assault with a weapon resulted in a lifetime PTSD diagnosis 9\% of the time; the sudden, unexpected death of someone close resulted in PTSD 31\% of the time; and involvement in a motor vehicle accident resulted in PTSD 2\% of the time. However, such a direct cause-and-effect relation was not found in the current study. 
In cases where assault with a weapon was the primary stressor, three of four PTSD diagnoses could be the result of a number of stressors. When the sudden unexpected death of someone close was the primary stressor, four of five PTSD diagnoses could have been the result of a number of stressors. And finally, when a motor vehicle accident was the primary stressor, three of four PTSD diagnoses could have been the result of a number of stressors. The only time this picture changes is when we consider combat, where four of five of the PTSD diagnoses were probably attributable to combat alone. However, as combat usually involves multiple traumatic experiences, this "discrete" stressor may also be a complex stressor.

Type I and Type II trauma. This review of the traumatic stressors experienced by inmates suggests that their PTSD symptoms are most likely attributable to multiple traumatic experiences. In the literature, a distinction is made between Type I and Type II trauma (Meichenbaum, 1994). Type I trauma is associated with discrete incidents such as motor-vehicle accidents, assault, and natural disasters. Type II trauma is associated with repeated exposure to traumatic events and is often experienced by groups such as police, firefighters, combat veterans, and the homeless. Meichbaum believes that Type II trauma is more likely to result in characterological and interpersonal problems than is Type I trauma.

Bessel van der Kolk (1996a) believes Type II trauma may lead to what he calls a disorder of extreme stress, not otherwise specified (DESNOS) (please see Appendix III for definition). Among the criteria suggested by van der Kolk for DESNOS are chronic affect dysregulation, difficulty modulating anger, self-destructive and suicidal behavior, difficulty modulating sexual involvement, impulsive and risk-taking behaviors, and chronic characterological changes, including an inability to trust and a tendency to victimize others. Anyone who has ever worked with inmates will find these criteria very familiar.

Therefore, current or past PTSD among inmates is usually the result of multiple rather than discrete stressors. Furthermore, these multiple stressors exacerbate and precipitate personality changes similar to those described by van der Kolk (1996a) for DESNOS. This means that the type of PTSD seen in inmates is not simply an Axis I 
disorder as defined by the DSM-IV (1994), but also carries with it elements of an Axis II disorder that may often warrant a diagnosis of personality disorder, not otherwise specified (NOS). In my opinion, a diagnosis of personality disorder, NOS, is preferable to a diagnosis of antisocial, narcissistic, or borderline personality disorder. This is because the personality disorder, NOS diagnosis allows the clinician greater latitude in the description of symptoms and avoids labeling that simply reinforces a stereotypical view of inmates.

Effectiveness of Diagnostic Instruments

When the current study was initiated, the researcher hoped it would be possible to identify an effective way of diagnosing PTSD within a correctional setting. To be effective, an instrument must be able to accurately identify current cases of PTSD (sensitivity), produce as few false positives as possible (specificity), and require a minimum of staff time to administer. As the CAPS-DX can take an hour or more to administer with individuals who are PTSD-positive, the time requirement points in the direction of a self-report measure. Both the Keane Scale of the MMPI-2 (PKS) and the Posttraumatic Diagnostic Scale (PDS ${ }^{\mathrm{TM}}$ ) only take 10-15 minutes to administer, so neither instrument places an undue burden on corrections staff. Therefore we will examine the performance of the PKS and PDSTM to determine if either is a good candidate for use as a PTSD screen in a correctional setting.

Keane Scale (PKS). The PKS was originally developed for use with Vietnam combat veterans (Keane, Malloy, \& Fairbank, 1984). In their study of the PKS standalone version, Herman, et al. (1996) identified a sensitivity of .82 and a specificity of .76 . In the current study, the PKS had a sensitivity of .64 and a specificity of .94 . This means that while the PKS correctly identified four-fifths of a combat veterans with current PTSD, it was only able to identify two-thirds of inmates with current PTSD. However, with inmates, the PKS did not produce a large number of false positives (five). Of these five false positives, four had non-current, lifetime PTSD according to the CAPS-DX. These numbers suggest that, although it performed well, the PKS is not as effective with an inmate population as it is with a combat population. 
Posttraumatic Stress Diagnostic Scale ${ }^{\mathrm{TM}}$ (PDSTM). The PDSTM was developed with a civilian population that had experienced a variety of potential traumatic stressors, including incarceration (Foa, et al. 1997). In the validation study conducted by Foa et al., the PDSTM was shown to have a sensitivity rating of 0.82 and a specificity of 0.77 . In the current study, the PDS ${ }^{\mathrm{TM}}$ demonstrated a sensitivity of 1.0 and a specificity of .84 . In other words, whereas it only identified four out of five cases of current PTSD in the validation study, it identified all cases of current PTSD in this study. However, the PDS $^{\mathrm{TM}}$ also identified 14 false positive cases, eight of which involved non-current, lifetime PTSD. Therefore, while the PDS ${ }^{T M}$ was just as good as the CAPS-DX at identifying current cases of PTSD within the inmate sample, men responding to it had a tendency to exaggerate current symptoms. But as $57 \%$ of those who exaggerated current symptomatology on the PDS ${ }^{\mathrm{TM}}$ had PTSD in the past, many of the false positives could be clinically useful.

Based on the results of the current study, it appears that PDS ${ }^{\mathrm{TM}}$ would be a more effective screening instrument with an inmate population than the PKS.

Recommendations for correctional use. The current study indicates that PTSD is more prevalent in a correctional setting than in the general population. PTSD has a high rate of comorbidity with major depression and substance abuse. Men with PTSD are often withdrawn, exhibit high levels of anger, and are less able to concentrate. Unresolved trauma may make it more difficult for these men to derive as much benefit as possible from prison educational programs. This, in turn, may lead to recidivism. Inmates may be unable to control their anger when unexpectedly reminded of past trauma. And they may be more likely to harm themselves, if they have comorbid major depression.

To help men get the most out of the educational opportunities that are offered to them, it is suggested that every inmate who is a candidate for a drug and alcohol program and a general equivalency program be asked to take the PDS ${ }^{\mathrm{TM}}$. A PDSTM result that indicates the presence of PTSD should not be used to exclude inmates from educational programs. It should be used as an indication that the inmate in question will also need to address trauma issues if he is to have the best chance of benefiting from the program in which he is involved. As a follow-up, inmates who appear to have current PTSD should 
then be administered both the Beck Depression Inventory (BDI) and the CAPS-DX. The BDI will serve as a screen for depression and potential self-harm, while the CAPS-DX will provide more detailed information about trauma symptoms, and this information will provide the foundation for an individualized treatment plan.

It is also recommended that any inmate who has spent time in SHU or on suicide watch be asked to complete the PDSTM. This will provide corrections psychologists with an efficient and effective way to determine if disruptive or self-destructive behavior is the result of PTSD. If it is, then the underlying trauma can be addressed, with a potential improvement in behavior management.

Group treatment. With current PTSD prevalence rates of $14 \%$, identification of inmates with the disorder could outstrip the ability to treat it effectively. Therefore, consideration should also be given to treating the problem in a group setting. van der Kolk, MacFarlane, and van der Hart (1996) note that the first part of such an effort should involve stabilization, which includes education about the disorder and identification of feelings. This is followed by deconditioning of the traumatic memories, restructuring of cognitions and schemata, social reconnection, and experiencing of corrective emotional experiences. Such a program could be combined with existing stress and anger management programs to enhance treatment effectiveness. Utilization of the group format would then allow staff to provide individual therapy for those inmates whose current PTSD is most severe.

Study Contributions, Limitations, and Future Directions

\section{$\underline{\text { Study Contributions }}$}

The current study has made the following contribution to the field's knowledge of PTSD: (a) Established both lifetime and current PTSD prevalence rates for federal inmates, (b) confirmed the lack of awareness of the presence of PTSD symptomatology by corrections psychologists, (c) provided new information about PTSD prevalence and minority status, (d) confirmed that incarceration can be a precipitant of PTSD symptomatology, (e) provided information suggesting that PTSD among inmates is usually the result of multiple, rather than discrete, stressors, ( $f$ ) set a benchmark for black and white participation rates for this type of study, (g) confirmed the important of 
multiple stressors to the development of PTSD symptomatology, and (h) identified a PTSD screening instrument for use in federal prisons.

\section{Study Limitations}

The site. The site chosen for this research study had an inmate population whose racial composition differed from the racial composition of the federal prison system as a whole. The federal prison system is $56.4 \%$ white, $40.1 \%$ black, and $3.5 \%$ other races. At the time of the study at FCI Cumberland, the inmate population was $64 \%$ black, 35\% white, and $1 \%$ other races. This means that even if a random sample drawn from this latter population was representative of the site itself, it would not be representative of the federal prison system as a whole. Therefore, a problem with generalizability existed even before the sample was drawn.

Overall participation. Fifty-three percent of inmates randomly selected for potential inclusion in this study ultimately agreed to participate in it. Three other studies involving inmates (Hodgins \& Cote, 1990; Teplin, 1990, 1996) had participation rates of 78\%, 95.4\%, and 95.8\%, respectively. In addition, Bureau of Prisons (BOP) personnel reported that participation rates in BOP research studies ranged from $55-80 \%$ for nonthreatening studies. However, the participants in Hodgins and Cote's study were exclusively French Canadian whites (S. Hodgins, personal communication, September 28, 1998); the inmates in Teplin's studies were paid for their participation; and the information on BOP studies was anecdotal. Therefore, it is not meaningful to compare participation rates in those studies with the participation rate in the current study.

However, Hodgins and Cote's (1990) participation rate of $78 \%$ compares favorably with the current study's participation rate of $71 \%$ for white inmates. Therefore, the current study has set the first participation rate benchmark for unpaid, incarcerated males of mixed race. Only after additional studies whose samples have similar characteristics have been conducted will it be possible to say whether or not the general participation rate in the current study is problematic.

The sample. The study's final sample ended up being $54 \%$ black, $44 \%$ white, and $2 \%$ other races. Therefore, it was not representative of either the federal population as a whole or the population at Cumberland. The sample's percentages fell between those of 
the local and national populations. As we have seen, the fact that the sample did not reflect the composition of the research site population was probably the result of a very low participation rate on the part of blacks. The lack of representativeness of the study's sample suggests that the results obtained must be utilized with caution.

The study's sample was not representative of the site because while $71 \%$ of whites agreed to participate, only $44 \%$ of blacks agreed to participate. One reason for this may have been the race and cultural characteristics of the sole researcher. Blacks may have been more reluctant to talk about a sensitive topic with a culturally-different researcher. Whatever the reason, the suppressed participation of blacks resulted in a sample that was not representative of the research site and may also have resulted in prevalence figures for blacks and whites that are not representative.

But this is all speculation. In fact, we know nothing about the motivation of participants and non-participants in this study. In addition to cross-cultural concerns, the decision to participate or not could have been influenced by a need to identify a reason for incarceration or past behavior, a desire to conceal a PTSD diagnosis, a potential participant's belief he was asymptomatic and would not contribute anything to the study, or a desire to help or hinder the researcher's efforts. All of these could have influenced participation and, therefore, sample composition. These are additional reasons these results should be evaluated with caution.

Use of a single researcher. The fact that all data for this study were collected by a single researcher also makes it difficult to gauge the accuracy of results derived from a structured clinical interview and, therefore, comparisons of it with self-report instruments. Because inter-rater reliability could not be computed, it is unclear whether there was a bias in the CAPS-DX assessments. If such a bias exists, then actual prevalence figures might be higher or lower than found. As sensitivity and specificity of the PKS and PDS ${ }^{\mathrm{TM}}$ results are also dependent on CAPS-DX, they may also be incorrect. However, the fact that nine of 14 current CAPS-DX diagnoses were confirmed by all three instruments and the remaining five by one other instrument suggests that there is little, if any, assessment bias. 
How limitations could be addressed. The facts that the sample is not representative of the entire federal inmate population, that inter-rater reliability could not be established, that participation rates could not be compared with other studies, and that participation rates differed significantly for blacks and whites, all suggest cautious evaluation of the results of this study. A larger sample, drawn from sites whose racial composition reflect that of the federal system as a whole, interviewed by numerous investigators of different races, sexes, and cultural backgrounds may increase confidence in future results.

\section{Future Directions}

A larger sample size. Due to the sample's lack of representativeness, it might be best to consider the identified rates of $30 \%$ lifetime PTSD and 14\% current PTSD as estimates. Undoubtedly, the results of the current study are robust enough to warrant further investigation. Such an investigation, involving more participants and sites that are reflective of the total federal inmate population, could provide a more definitive answer concerning overall prevalence, as well as prevalence rates manifested by different racial groups. It might also provide better information concerning individual characteristics and life experiences that may suggest the presence of PTSD.

Varied racial composition of sample sites. Even though the overall pool from which the sample is drawn needs to reflect the BOP population as a whole, subsets of that pool could reflect varying local minority and majority status. For example, one site might have a majority of blacks, another a majority of whites, and still another a majority of Hispanics. Analysis of the results obtained at these different sites could expand our understanding of whether or not symptoms of mental disorders vary according to local minority status.

A varied research team. In order to achieve a greater degree of certainty concerning results, the investigative team needs to be culturally diverse, and an attempt should be made to match researchers with research participants. These research teams need to include African-American, Caucasian, and Hispanic members. It would also be important to include female investigators, as a recent study by Pollner (1998) suggests that female interviewers may create an atmosphere that is more conducive to self- 
disclosure. With a large-enough sample, enough research cells can be created to determine whether or not cultural matching has an impact on results.

The importance of inultiple stressors. The current study strongly suggests that the greater the number of traumatic stressors experienced by an inmate, the more PTSD symptomatology he will experience. In re-visiting the question of stressors, the emphasis needs to be placed on the total number of events experienced by inmates that satisfy Criterion A. Such an investigation might provide support for the idea that inmates are more likely to experience PTSD as the result of multiple traumatic stressors than as the result of a single, discrete stressor. The stressor study could be carried out separately from a more definitive prevalence study.

The presence of complex PTSD symptomatology. It would also be interesting to determine whether or not van der Kolk's (1996a) description of DESNOS is more applicable to a prison population than the DSM-IV's (1994) diagnostic criteria for PTSD. As inmates have experienced so many different types of stressors, and as these stressors are often prolonged in nature, the population of inmates may be an excellent one in which to examine the DESNOS diagnosis. It is also possible that Teplin, Abram, and McClelland's (1996) identified lifetime PTSD rate of 33\% for female inmates includes a number of complex PTSD cases. Therefore, female inmates should be included in this type of study. If this is done, then researchers can also determine if and/or how the symptom patterns of the two groups are similar.

\section{Summary}

The current study identified a lifetime PTSD prevalence rate of $30 \%$ and a current rate of $14 \%$ among males incarcerated in a federal prison. Although there were no prevalence rate differences between men in minimum and medium security, whites manifested a higher rate of PTSD (41\%) than did blacks (22\%), and much greater PTSD symptomatology than blacks when in the minority. Although none of the men with current PTSD and only one with lifetime PTSD was identified by corrections psychologists as having the disorder, five of 14 men with current PTSD were identified as having an anxiety or mood disorder. Even though IQ and SES did not turn out to be predictors of PTSD in this study, the number of potential stressors experienced by an 
inmate, the number of siblings he had, and a prior diagnosis of a mental disorder were identified as significant predictors of PTSD. Of the three, the number of stressors experienced was the most important. The study also made it clear that incarceration itself can be a precipitant of PTSD. However, most inmates who identified it as an immediate precipitant were, in fact, responding to multiple stressors. Along these lines, it appears as though the majority of PTSD cases in this population may be of a complex nature. The study also identified the PDSTM as an effective PTSD screening instrument, that could help corrections psychologists identify current PTSD in a cost-effective manner.

Subsequent treatment of those so identified could result in improved management of the inmate population and a reduction in recidivism.

The study's major limitation is the sample's lack of congruence with the research site itself and the federal prison system, as a whole. This was the result of the research site's not being reflective of the federal system and of the low participation rate on the part of blacks. Future studies could increase confidence in the prevalence rates obtained by increasing the sample size, by making sure the sample is reflective of the federal inmate population as a whole, and by making the investigative team culturally and sexually diverse. The last change would also solve the problem of a lack of inter-rater reliability in the current study. Finally, research could be conducted concerning the impact of multiple stressors on male and female inmates. Due to the fact that most inmates have experienced multiple stressors, research with them might provide new insight into the appropriateness of the DESNOS diagnosis.

However, the study's main finding of a lifetime prevalence rate of $30 \%$ is robust enough to suggest that despite methodological weaknesses there is a difference between the rate of PTSD in the general population and the rate among federal prison inmates. 


\section{References}

Albrecht, N. N., Talbert, F. S., Albrecht, J. W., Boudewyns, P. A., Hyer, L. A., Touze, J., \& Lemmon, C. R. (1994). A comparison of MMPI and MMPI-2 in PTSD assessment. Journal of Clinical Psychology, 50, 578-585.

Allen, S. N. (i $9 \% 4$ ). Psychological assessment of post-traumatic stress disorder: Psychometrics, currents trends, and future directions. Psychiatric Clinics of North America, 17, 327-349.

American Psychiatric Association. (1980). Diagnostic and statistical manual of mental disorders (3rd ed.). Washington, DC: Author.

American Psychiatric Association. (1987). Diagnostic and statistical manual of mental disorders (3rd ed., rev.). Washington, DC: Author.

American Psychiatric Association. (1994). Diagnostic and statistical manual of mental disorders (4th ed.). Washington, DC: Author.

Anastasi, A. (1988). Psychological testing (6th ed.). New York: Macmillan.

Andersen, H. S., Christensen, A. K., \& Petersen, G. O. (1991). Post-traumatic stress reactions amongst rescue workers after a major rail accident. Anxiety Research, 4 , 245-251.

Ben-Porath, Y. S., \& Butcher, J. N. (1989). Psychometric stability of rewritten MMPI items. Journal of Personality Assessment, 53, 645-653.

Blake, D. D., Weathers, F. W., Nagy, L. M., Kaloupek, D. G., Gusman, F. D., Charney, D. S., \& Keane, T. M. (1995). The development of a clinician-administered PTSD scale. Journal of Traumatic Stress, 8, 75-90.

Blake, D. D., Weathers, F. W., Nagy, L. M., Kaloupek, D. G., Klauminzer, G., Charney, D. S., \& Keane, T. M. (1990). A clinician rating scale for assessing current and lifetime PTSD: The CAPS-1. The Behavior Therapist, 13, 187-188.

Blanchard, E. B., Hickling, E. J., Taylor, A. E., Loos, W. R., \& Gerardi, R. J. (1994). Psychological morbidity associated with motor vehicle accidents. Behaviour Research and Therapy, 32, 283-290.

Breslau, N., Davis, G. C., \& Andreski, P. (1995). Risk factors for PTSD-related traumatic events: A prospective analysis. American Journal of Psychiatry, 152, 529-535. 
Breslau, N., Davis, G. C., Andreski, P., \& Peterson, E. (1991). Traumatic events and posttraumatic stress disorder in an urban population of young adults. Archives of General Psychiatry, 48, 216-222.

Breslau, N., Kessler, R. C., Chilcoat, H. D., Schultz, L. R., Davis, G. C., \& Andreski, P. (1998). Trauma and posttraumatic stress disorder in the community. Archives of General Psychiatry, 55, 626-632.

Briere, J. (1997). Psychological assessment of adult posttraumatic states. Washington, DC: American Psychological Association.

Burstein, A. (1989). Posttraumatic stress disorder in victims of motor vehicle accidents. Hospital and Community Psychiatry, 40, 295-297.

Caldwell, M. F. (1992). Incidence of PTSD among staff victims of patient violence. Hospital and Community Psychiatry, 43, 838-839.

Collins, J. J., \& Bailey, S. L. (1990). Traumatic stress disorder and violent behavior. Journal of Traumatic Stress, 3, 203-220.

Cote, G., \& Hodgins, S. (1990). Co-occurring mental disorders among criminal offenders. Bulletin of the American Academy of Psychiatry and the Law, 18, 271- 281.

Cottler, L. B., Compton, W. M., Mager, D., Spitznagel, E. L., \& Janca, A. (1992).

Cottler, L. B., Compton, W. M., Mager, D., Spitznagel, E. L., \& Janca, A. (1992). Posttraumatic stress disorder among substance users from the general population. American Journal of Psychiatry, 149, 664-670.

Daniel, A. E., Robins, A. J., Reid, J. C., \& Wilfley, D. E. (1988). Lifetime and six-month prevalence of psychiatric disorders among sentenced female offenders. Bulletin of the American Academy of Psychiatry and the Law, 16, 333-342.

Davidson, J. R. T., \& Fairbank, J. A. (1993). The epidemiology of postraumatic stress disorder. In J. R. T. Davidson \& E. B. Foa (Eds.), Posttraumatic stress disorder: DSM IV and beyond (pp. 147 - 169). Washington, DC: American Psychiatric Press.

Davidson, J. R. T., Hughes, D., Blazer, D. G., \& George, L. K. (1991). Post-traumatic stress disorder in the community: An epidemiological study. Psychological Medicine, 21, 713-721. 
Davis, L. E. (1979). Racial composition of groups. Social Work, 24, 208-213.

Davis, G. C., \& Breslau, N. (1994). Post-traumatic stress disorder in victims of civilian trauma and criminal violence. Psychiatric Clinics of North America, 17, 289-299.

Dunn, G. E., Paolo, A. M., Ryan, J. J., \& Van Fleet, J. (1993). Dissociative symptoms in a substance abuse population. American Journal of Psychiatry, 150, 1043-1047.

Dvoskin, J. A., \& Steadman, H. J. (1989). Chronically mentally ill inmates: The wrong concept for the right services. International Journal of Law and Psychiatry, 12. 203-210.

Fairbank, J. A., McCaffrey, R. J., \& Keane, T. M. (1985). Psychometric detection of fabricated symptoms of post-traumatic stress disorder. American Journal of Psychiatry. 142, 501-503.

Foa, E. B. (1995). Posttraumatic Stress Diagnostic Scale ${ }^{\mathrm{TM}}$ : Manual. Minneapolis, MN: National Computer Systems, Inc.

Foa, E. B., Cashman, L., Jaycox, L., \& Perry, K. (1997). The validation of a self-report measure of posttraumatic stress disorder: The Posttraumatic Diagnostic Scale. Psychological Assessment, 9, 445-451.

Foa, E. B., Riggs, D. S., \& Gershuny, B. S. (1995). Arousal, numbing, and intrusion: Symptom structure of PTSD following assault. American Journal of Psychiatry, 152. 116-120.

Foa, E. B., Rothbaum, B. O., \& Steketee, G. S. (1993). Treatment of rape victims. Journal of Interpersonal Violence, 8, 256-276.

Follette, V. M., Polusny, M. M., \& Milbeck, K. (1994). Mental health and law enforcement professionals: Trauma history, psychological symptoms, and impact of providing services to child sexual abuse survivors. Professional Psychology: Research and Practice, 25, 275-282.

Friedman, M. J., Schnurr, P. P., \& McDonagh-Coyle, A. (1994). Post-traumatic stress disorder in the military veteran. Psychiatric Clinics of North America, 17, $265-$ 277. 
Fullerton, C. S., McCarroll, J. E., Ursano, R. J., \& Wright, K. M. (1992). Psychological responses of rescue workers: Fire fighters and trauma. American Journal of Orthopsychiatry, 62, 371-378.

Graham, J. R. (1993). MMPI-2: Assessing personality and psychopathology (2nd ed.). New York: Oxford University Press.

Green, B. L. (1994). Psychosocial research in traumatic stress: An update. Journal of Traumatic Stress, 7, 341-362.

Green, B. L., Epstein, S. A., Krupnick, J. L., \& Rowland, J. H. (1997). Trauma and medical illness: Assessing trauma-related disorders in medical settings. In J. P. Wilson \& T. M. Keane (Eds.), Assessing psychological trauma and PTSD (pp. 160-191). New York: Guilford.

Green, B. L., \& Lindy, J. D. (1994). Post-traumatic stress disorder in victims of disaster. Psychiatric Clinics of North America, 17, 301-309.

Green, B. L., Lindy, J. D., Grace, M. C., Gieser, G. C., Leonard, A. C., Korol, M., \& Winget, C. (1990). Buffalo Creek survivors in the second decade: Stability of stress symptoms. American Journal of Orthopsychiatry, 60, 43-54.

Halpern, D. (1993). Minorities and mental health. Social Science and Medicine, 36, 597607.

Harvey, M. R., \& Herman, J. J. (1992). The trauma of sexual victimization. PTSD Research Quarterly, 3, 1-7.

Helzer, J. E., Robins, L. N., \& McEvoy, L. (1987). Post-traumatic stress disorder in the general population: Findings of the epidemiologic catchment area survey. The

New England Journal of Medicine, 317, 1630-1634.

Helzer, J. E., Spitznagel, E. L., \& McEvoy, L. (1987). The predictive validity of lay diagnostic interview schedule diagnoses in the general population. Archives of General Psychiatry, 44, 1069-1077. 
Herman, D. S., Weathers, F. W., Litz, B. T., \& Keane, T. M. (1996). Psychometric properties of the embedded and stand-alone versions of the MMPI-2 Keane PTSD scale. Psychological Assessment, 3, 437-442.

Hodge, J. E. (1992). Addiction to violence: A new model of psychopathology. Criminal Behaviour and Mental Health, 2, 212-223.

Hodgins, S. (1993). The criminality of mentally disordered persons. In S. Hodgins (Ed.), Mental disorder and crime (pp. 3-21). Newbury Park, CA: Sage.

Hodgins, S. (1995). Assessing mental disorder in the criminal justice system: Feasibility vs. clinical accuracy. International Journal of Law and Psychiatry, 18. 15-28.

Hodgins, S., \& Cote, G. (1990). Prevalence of mental disorders among penitentiary inmates in Quebec. Canada's Mental Health, 38(1), 1-4.

Hodgins, S., \& Cote, G. (1993). The criminality of mentally disordered offenders. Criminal Justice and Behavior, 20, 115-129.

Hollingshead, A. B. (1975). Four factor index of social position. Unpublished manuscript. New Haven, CT: Yale University.

Howell, D. C. (1992). Statistical methods for psychology (3rd ed.). Belmont, CA: Duxbury Press.

Hyer, L., Summers, M. N., Braswell, L., \& Boyd, S. (1995). Posttraumatic stress disorder: Silent problem among older combat veterans. Psychotherapy, 32, 348364.

Kaufman, A. S. (1990). Assessing adolescent and adult intelligence. Boston: Allyn and Bacon.

Kaufman, A. S., Ishikuma, T. \& Kaufman-Packer, J. L. (1991). Amazingly short forms of the WAIS-R. Journal of Psychoeducational Assessment, 9, 4-15.

Keane, T. M., Malloy, P. F., \& Fairbank, J. A. (1984). Empirical development of an MMPI subscale for the assessment of combat-related posttraumatic stress disorder. Journal of Consulting and Clinical Psychology, 52, 888-891. 
Keane, T. M., \& Wolfe, J. (1990). Comorbidity in post-traumatic stress disorder: An analysis of community and clinical studies. Journal of Applied Social Psychology, 20, 1776-1788.

Keppel, G. (1991). Design and analysis: A researcher's handbook (3rd ed.). Engelwood Cliffs, NJ: Prentice Hall.

Kessler, R. C., Sonnega, A., Bromet, E., Hughes, M., \& Nelson, C. B. (1995).

Posttraumatic stress disorder in the national comorbidity survey. Archives of General Psychiatry, 52, 1048-1060.

Kilpatrick, D. G., Saunders, B. E., Veronen, L. J., Best, C. L., \& Von, J. M. (1987).

Criminal victimization: Lifetime prevalence, reporting to police, and psychological impact. Crime and Delinquency, 33, 479-489.

Kirk, R. E. (1995). Experimental design: Procedures for the behavioral sciences. New York: Brooks/Cole Publishing Company.

Kolb, L. C. (1989a). Heterogeneity of PTSD [Letter to the editor]. American Journal of Psychiatry, 146, 811-812.

Kolb, L. C. (1989b). Chronic post-traumatic stress disorder: Implications of recent epidemiological and neuropsychological studies. Psychological Medicine, 19. 821-824.

Koretz, M. B., \& Peck, A. H. (1990). Validation and cross-validation of the PTSD subscale of the MMPI with civilian trauma victims. Journal of Clinical

Psychology, 46, 296-300.

Kulka, R. A., Schlenger, W. E., Fairbank, J. A., Hough, R. L., Jordan, Marmar, C. R., \& Weiss, D. S. (1990). Trauma and the Vietnam war generation: Report of findings from the National Vietnam Veterans Readjustment Study. New York: Brunner/Mazel.

Kulka, R. A., Schlenger, W. E., Fairbank, J. A., Jordan, B. K., Hough, R. L., Marmar, C. R., \& Weiss, D. S. (1991). Assessment of posttraumatic stress disorder in the community: Prospects and pitfalls from recent studies of Vietnam Veterans. Psychological Assessment: A Journal of Consulting and Clinical Psychology, 3. 547-560. 
Kutz, I., Garb, R., \& David, D. (1988). Post-traumatic stress disorder following myocardial infarction. General Hospital Psychiatry, 10 , 169-176.

Lindqvist, P., \& Allebeck, P. (1990). Schizophrenia and crime: A longitudinal follow-up of 644 schizophrenics in Stockholm. British Journal of Psychiatry, 157, 345-350.

Link, B. G., Andrews, H., \& Cullen, F. T. (1992). The violent and illegal behavior of mental patients reconsidered. American Sociological Review, 57, 275-292.

Litz, B. T., Penk, W. E., Walsh, S., Hyer, L., Blake, D. B., Marx, B., Keane, T. M., \& Bitman, D. (1991). Similarities and differences between MMPI and MMPI-2 applications to the assessment of postraumatic stress disorder. Journal of Personality Assessment, 57, 238-253.

Lyons, J. A., \& Keane, T. M. (1992). Keane PTSD scale: MMPI and MMPI-2 update. Journal of Traumatic Stress. 5, 111-117.

Lyons, J. A., \& Scotti, J. R. (1994). Comparability of two administration formats of the Keane Posttraumatic Stress Disorder scale. Psychological Assessment, 6, 209211.

Madakasira, S., \& O'Brien, K. F. (1987). Acute posttraumatic stress disorder in victims of a natural disaster. Journal of Nervous and Mental Disease, 175, 286-290.

Manson, S. M. (1997). Cross-cultural and multiethnic assessment of trauma. In J. P. Wilson, \& T. M. Keane (Eds.), Assessing psychological trauma and PTSD (pp. 239-266). New York: Guilford.

Martin, C. A., McKean, H. E., \& Veltkamp, L. J. (1986). Post-traumatic stress disorder in police and working with victims. Journal of Police Science and Administration, 14. 98-101.

McCafferty, F. L., McCafferty, E., \& McCafferty, M. A., (1992). Stress and suicide in police officers: Paradigm of occupational stress. Southern Medical Journal, 85, 233-243.

McCaffrey, R. J., Hickling, E. J., \& Marrazo, M. J. (1989). Civilian-related posttraumatic-stress disorder: Assessment-related issues. Journal of Clinical Psychology, 45, 72-76. 
McCranie, E. W., Hyer, L. A., Boudewyns, P. A., \& Woods, M. G. (1992). Negative parenting behavior, combat exposure, and PTSD symptom severity. Joumal of Nervous and Mental Disease, 180, 431-438.

McFarlane, A. C. (1988). The phenomenology of posttraumatic stress disorders following a natural disaster. Journal of Nervous and Mental Disease, 176, 22-29.

McFarlane, A. C., \& de Girolamo, G. (1996). The nature of traumatic stressors and the epidemiology of posttraumatic reactions. In B. van der Kolk, A. C. McFarlane, \& L. Weisaeth (Eds.), Traumatic stress: The effects of overwhelming experience on mind, body, and society (pp. 129-154). New York: Guilford.

McFarlane, A. C., \& Papay, P. (1992). Multiple diagnoses in posttraumatic stress disorder in the victims of a natural disaster. Journal of Nervous and Mental Disease, 180 , 498-504.

McFarlane, A. C., \& Yehuda, R. (1996). Resilience, vulnerability, and the course of posttraumatic reactions. In B. van der Kolk, A. C. McFarlane, \& L. Weisaeth (Eds.), Traumatic stress: The effects of overwhelming experience on mind, body. and society (pp. 155-181). New York: Guilford.

McNally, R. J., \& Shin, L. M. (1995). Association of intelligence with severity of posttraumatic stress disorder symptoms in Vietnam combat veterans. American Journal of Psychiatry, 152, 936-938.

Meichenbaum, D. (1994). A clinical handbook/practical therapist manual for assessing and treating adults with post-traumatic stress disorder (PTSD). Waterloo, Canada: Institute Press.

Nader, K., Pynoos, R., Fairbanks, L., \& Frederick, C. (1990). Children's PTSD reactions one year after a sniper attack at their school. American Journal of Psychiatry, 147. 1526-1530.

Newman, E., Kaloupek, D. G., \& Keane, T. M. (1996). Assessment of posttraumatic stress disorder in clinical and research settings. In B. van der Kolk, A. C. McFarlane, \& L. Weisaeth (Eds.), Traumatic stress: The effects of overwhelming experience on mind, body, and society (pp. 242-275). New York: Guilford. 
Norris, F. H. (1992). Epidemiology of trauma: Frequency and impact of different potentially traumatic events on different demographic groups. Journal of Consulting and Clinical Psychology, 60, 409-418.

North, C. S., \& Smith, E. M. (1992). Posttraumatic stress disorder among homeless men and women. Hospital and Community Psychiatry, 43, 1010-1016.

Norusis, M. J. (1995). SPSS 6.1: Guide to data analysis. Englewood Cliffs, NJ: Prentice Hall.

Ogloff, J. R. P., Roesch, R., \& Hart, S. D. (1993). Screening, assessment, and identification of services for mentally ill offenders. In H. J. Steadman \& J. J. Cocozza (Eds.) Mental illness in America's prisons (pp. 61-90). Seattle, WA: National Coalition for the Mentally $\mathrm{Il}$ in the Criminal Justice System.

Pollner, M. (1998). The effects of interviewer gender in mental health interviews. Journal of Nervous and Mental Disease, 186, 369-373.

Post, R. M. (1992). Transduction of psychosocial stress into the neurobiology of recurrent affective disorder. American Journal of Psychiatry, 149, 999-1010.

Resnick, H., Yehuda, R., Pitman, R. K., \& Foy, D. W. (1995). Effect of previous trauma on acute plasma cortisol level following rape. American Journal of Psychiatry, 152. 1675-1677.

Robins, L. N., Helzer, J. E., Weissman, M. M., Orvaschel, H., Gruenberg, E., Burke, J. D., Jr., \& Regier, D. A. (1984). Lifetime prevalence of specific psychiatric disorders in three sites. Archives of General Psychiatry, 41, 949-958.

Roesch, R., Ogloff, J. R. P., \& Eaves, D. (1995). Mental health research in the criminal justice system: The need for common approaches and international perspectives. International Journal of Law and Psychiatry, 18, 1-14.

Ross, C. A., Anderson, G., Heber, S., \& Norton, G. R. (1990). Dissociation and abuse among multiple-personality patients, prostitutes, and exotic dancers. Hospital and Community Psychiatry, 41, 328-330.

Rubin, P. N., \& McCampbell, S. W. (1995). The Americans with Disabilities Act and criminal justice: Mental disabilities and corrections. Washington, DC: US Department of Justice. 
Saxe, G. N., van der Kolk, B. A., Berkowitz, R., Chinman, G., Hall, K., Lieberg, G., \& Schwartz, J. (1993). Dissociative disorders in psychiatric inpatients. American Journal of Psychiatry, 150, 1037-1042.

Scotti, J. R. (in press). Clinical assessment of posttraumatic stress disorder. In Handbook of psychiatric measures and outcomes. Washington, DC: American Psychiatric Press.

Shalev, A. Y. (1996). Stress versus traumatic stress: From acute homeostatic reactions to chronic psychopathology. In B. van der Kolk, A. C. McFarlane, \& L. Weisaeth (Eds.), Traumatic stress: The effects of overwhelming experience on mind, body, and society (pp. 77-101). New York: Guilford.

Shalev, A. Y., Peri, T., Canetti, L., \& Schreiber, S. (1996). Predictors of PTSD in injured trauma survivors: A prospective study. American Journal of Psychiatry, 153, 219225.

Shore, J. H., Vollmer, W. M., \& Tatum, E. L. (1989). Community patterns of postraumatic stress disorder. Journal of Nervous and Mental Disease, 177, 681685.

Sloan. P. (1988). Post-traumatic stress disorder in survivors of an airplane crash-landing: A clinical and exploratory research intervention. Journal of Traumatic Stress, 1 , 211-229.

Solomon, Z., Waysman, M., Levy, G., Fried, B., Mikulincer, M., Benbenishty, R., Florian, V., \& Bleich, A. (1992). From front line to home front: A study of secondary traumatization. Family Process, 31, 289-302.

Steadman, H. J., Fabisiak, S., Dvoskin, J., \& Holohean, E. J. (1987). A survey of mental disability among state prison inmates. Hospital and Community Psychiatry, 38 , 1086-1090.

Stein, Z., \& Susser, M. (1981). Methods in epidemiology. American Academy of Child Psychiatry, 20, 444-461.

Steinglass, P., \& Gerrity, E. (1990). Natural disasters and post-traumatic stress disorder: Short-term versus long-term recovery in two disaster-affected communities. Journal of Applied Social Psychology, 20, 1746-1765. 
Sue, S., Zane, N., \& Young, K. (1994). Research on psychotherapy with culturally diverse populations. In A. E. Bergin, \& S. L. Garfield (Eds.), Handbook of psychotherapy and behavior change (4th Ed.). New York: Wiley.

Teplin, L. A. (1984). Criminalizing mental disorder: The comparative arrest rate of the mentally ill. American Psychologist, 39, 794-803.

Teplin, L. A. (1990a). Detecting disorder: The treatment of mental illness among jail detainees. Journal of Consulting and Clinical Psychology, 58, 233-236.

Teplin, L. A. (1990b). The prevalence of severe mental disorder among male urban jail detainees: Comparison with the epidemiologic catchment area program. American Journal of Public Health, 80, 663-669.

Teplin, L. A., Abram, K. M., \& McClelland, G. M. (1996). Prevalence of psychiatric disorders among incarcerated women: I. Pretrial jail detainees. Archives of General Psychiatry, 53, 505-512.

Thoits, P. (1983). Dimensions of life events that influence psychological distress: An evaluation and synthesis of the literature. In H. B. Kaplan (Ed.), Psychological stress: Trends in Theory and Research. New York: Academic Press.

True, W. R., Rice, J., Eisen, S. A., Heath, A. C., Goldberg, J., Lyons, M. J., \& Nowak, J. (1993). A twin study of genetic and environmental contributions to liability for posttraumatic stress symptoms. Archives of General Psychiatry, 50, 257-264.

US Department of Justice. (1994a). State of the bureau. Washington, DC: Author.

US Department of Justice. (1994b). National corrections reporting program, 1992.

NCJ-145862. Washington, DC: Author.

US Department of Justice. (1995a). State of the bureau. Washington, DC: Author.

US Department of Justice. (1995b). Prisoners in 1994. NCJ-151654. Washington, DC: Author.

van der Kolk, B. A. (1995). Counting the cost: The lasting impact of childhood trauma. Nevada City, CA: Cavalcade Productions.

van der Kolk, B. A. (1996a). The body keeps the score: Approaches to the psychobiology of posttraumatic stress disorder. In B. A. van der Kolk, A. C. McFarlane, \& L. 
Weisaeth (Eds.), Traumatic stress: The effects of overwhelming experience on mind, body, and society (pp. 214-241). New York: Guilford.

van der Kolk, B. A. (1996b). The complexity of adaptation to trauma: Self-regulation, stimulus discrimination, and characterological development. In B. A. van der Kolk, A. C. McFarlane, \& L. Weisaeth (Eds.), Traumatic stress: The effects of overwhelming experience on mind, body, and society (pp. 182-213). New York: Guilford.

van der Kolk, B. A., \& McFarlane, A. C. (1996). The black hole of trauma. In B. A. van der Kolk, A. C. McFarlane, \& L. Weisaeth (Eds.), Traumatic stress: The effects of overwhelming experience on mind, body, and society (pp. 3-23). New York: Guilford.

van der Kolk, B. A., McFarlane, A. C., \& van der Hart, O. (1996). A general approach to treatment of posttraumatic stress disorder. In B. A. van der Kolk, A. C. McFarlane, \& L. Weisaeth (Eds.), Traumatic stress: The effects of overwhelming experience on mind, body, and society (pp. 417-440). New York: Guilford. van der Kolk, B. A., Pelcovitz, D., Roth, S., Mandel, F. S., McFarlane, A. C., \& Herman, J. A. (1996). Dissociation, somatization, and affect dysregulation: The complexity of adaptation to trauma. American Journal of Psychiatry, 153, 83-93.

Vrana, S., \& Lauterbach, D. (1994). Prevalence of traumatic events and post-traumatic psychological symptoms in a nonclinical sample of college students. Journal of Traumatic Stress, 7, 289-302.

Weathers, F. W., \& Litz, B. T. (1994). Psychometric properties of the ClinicianAdministered PTSD Scale, CAPS-1. PTSD Research Quarterly, 5(2), 2-6. Wechsler, D. (1981). WAIS-R manual. New York: Harcourt-Brace-Jovanovich. Weiss, D. S. (1993). Structured clinical interview techniques. In J. P. Wilson \& B. Raphael (Eds.), International handbook of posttraumatic stress syndromes (pp. 179-187). New York: Plenum.

Weiss, D. S., Marmar, C. R., Schlenger, W. E., \& Fairbank, J. A. (1992). The prevalence of lifetime and partial post-traumatic stress disorder in Vietnam theater veterans. Journal of Traumatic Stress, 5, 365-376. 
Yehuda, R., \& McFarlane, A. C. (1995). Conflict between current knowledge about posttraumatic stress disorder and its original conceptual base. American Journal of Psychiatry, 152, 1705-1713.

Zaidi, L. Y., \& Foy, D. W. (1994). Childhood abuse experiences and combat-related PTSD. Journal of Traumatic Stress, 7, 33-42. 
Appendix A: DIAGNOSTIC CRITERIA

\title{
Diagnostic Criteria for 309.81 Posttraumatic Stress Disorder
}

\begin{abstract}
A. The person has been exposed to a traumatic event in which both of the following were present:
\end{abstract}

(1) the person experienced, witnessed, or was confronted with an event or events that involved actual or threatened death or serious injury, or a threat to the physical integrity or self or others

(2) the person's response involved intense fear, helplessness, or horror. Note: In children, this may be expressed instead by disorganized or agitated behavior.

B. The traumatic event is persistently reexperienced in one (or more) of the following ways:

(1) recurrent and intrusive distressing recollections of the event, including images, thoughts, or perceptions. Note: In young children, repetitive play may occur in which themes or aspects of the trauma are expressed

(2) recurrent distressing dreams of the event. Note: In children, there may be frightening dreams without recognizable content

(3) acting or feeling as if the traumatic event were recurring (includes a sense of reliving the experience, illusions, hallucinations, and dissociative flashback episodes, including those that occur on awakening or when intoxicated. Note: In young children, trauma-specific reenactment may occur

(4) intense psychological distress at exposure to internal or external cues that symbolize or resemble an aspect of the traumatic event

(5) physiological reactivity on exposure to internal or external cues that symbolize or resemble an aspect of the traumatic event

C. Persistent avoidance of stimuli associated with the trauma and numbing of general responsiveness (not present before the trauma), as indicated by three (or more) of the following:

(1) efforts to avoid thoughts, feelings, or conversations associated with the trauma

(2) efforts to avoid activities, places, or people that arouse recollections of the trauma 
(3) inability to recall an important aspect of the trauma

(4) marked diminished interest or participation in significant activities

(5) feeling of detachment or estrangement from others

(6) restricted range of affect (e.g. unable to have loving feelings)

(7) sense of a foreshortened future (e.g., does not expect to have a career, marriage, children, or a normal life span)

D. Persistent symptoms of increased arousal (not present before the trauma), as indicated by two (or more) of the following:

(1) difficulty falling or staying asleep

(2) irritability or outbursts of anger

(3) difficulty concentrating

(4) hypervigilance

(5) exaggerated startle response

E. Duration of the disturbance (symptoms in Criteria B. C. and D) is more than 1 month.

F. The disturbance causes clinically significant distress or impairment in social, occupational, or other important areas of functioning.

Specify if:

Acute: if duration of symptoms is less than 3 months

Chronic: if duration of symptoms is 3 months or more Specify if:

With delayed onset: if onset of symptoms is at least 6 months after the stressor Source: DSM-IV (American Psychiatric Association, 1994) 
Proposed Criteria: Disorders of Extreme Stress Not Otherwise Specified (DESNOS)

A. Alterations in regulating affective arousal

(1) chronic affect dysregulation

(2) difficulty modulating anger

(3) self-destructive and suicidal behavior

(4) difficulty modulating sexual involvement

(5) impulsive and risk-taking behaviors

B. Alterations in attention and consciousness

(1) amnesia

(2) dissociation

C. Somatization

D. Chronic characterological changes

(1) alterations in self-perception: chronic guilt and shame; feelings of self-blame, of ineffectiveness, and of being permanently damaged

(2) alterations in perception of perpetrator: adopting distorted beliefs and idealizing the perpetrator

(3) alterations in relations with others:

(a) an inability to trust or maintain relationships with others

(b) a tendency to be revictimized

(c) a tendency to victimize others

E. Alterations in systems of meaning

(1) despair and hopelessness

(2) loss of previously sustaining beliefs

Source: van der Kolk, B. A., McFarlane, A. C., \& Weisaeth, L. (Eds.) (1996). Traumatic stress: The effects of overwhelming experience on mind, body, and society. New York: Guilford Press. 


\section{Appendix B: RESEARCH QUESTIONNAIRE}

The Research Questionnaire is reproduced here. The Keane Scale of the MMPI-2, the Posttraumatic Stress Diagnostic Scale ${ }^{\mathrm{TM}}$, and the WAIS-R subtests utilized in this research are all protected materials. Therefore, none of them are reproduced in this appendix. Information on obtaining the Life Events Checklist and the CAPS-DX will be provided by the author on request. 


\section{CONFI DENTIAL}

Research Questionnaire

Please answer the following questions to the best of your ability.

Date of Birth: \# of Years Incarcerated:

Race: ( ) Asian ( ) Black ( ) Hispanic ( ) Native American ( ) White ( )Other

Native Language:

Religion:

Do you practice your religion?

( ) Yes ( ) No

Father's

Father's

Occupation: Education:

Mother's

Occupation:

Education:

Parents marital status when you were growing up:

How many children were there in your family? What number were you?

Are you a high school graduate? ( ) Yes ( ) No If no, what grade did you complete

Did you attend college?

( ) Yes ( ) No If yes, how many years

What is your marital status?

( ) Single

( ) Married

( ) Divorced

( ) Widower

How many times have you been married?

How many children do you have?

What is your primary occupation?

Were you in the military?

( ) Yes ( ) No If yes, were you in combat?

( ) Yes ( ) No

Have you ever received treatment for any mental disorder?

( ) Yes ( ) No

If yes, what was the disorder?

Have you ever been told you had posttraumatic stress disorder (PTSD)?

( ) Yes ( ) No

If yes, who told you you had it?

Have you been addicted to or dependent on drugs?

( ) Yes () No

Alcohol? ( ) Yes ( ) No

Have you ever been charged with a violent crime?

( ) Yes ( ) No

If yes, what was the crime? 


\section{ABSTRACT \\ THE PREVALENCE OF \\ POSTTRAUMATIC STRESS DISORDER \\ AMONG FEDERAL PRISON INMATES}

\section{By Robert K. Guthrie}

Epidemiological studies concerning the prevalence of posttraumatic stress disorder (PTSD) have identified lifetime rates for males ranging from $0.5 \%$ to $6 \%$. By contrast, studies of prison inmates have identified lifetime prevalence rates for PTSD ranging from $2.3 \%$ to $13 \%$. The central hypothesis of this study stated that prevalence rates for incarcerated males are higher than previously identified. In the study, 100 federal inmates were assessed for PTSD using the Clinician-Administered PTSD Scale-DX (CAPS-DX), the Posttraumatic Stress Diagnostic Scale ${ }^{\mathrm{TM}}$ (PDS ${ }^{\mathrm{TM}}$ ), and the Keane Scale from the MMPI-2. Using the CAPS-DX as the official diagnostic instrument, a lifetime prevalence rate of $30 \%$ and a current prevalence rate of $14 \%$ were identified. Both rates were significantly higher than previously identified rates. Corrections psychologists were unaware of the presence of all but one PTSD case identified in the study. The PDS ${ }^{\mathrm{TM}}$ was identified as an effective screening instrument for use with federal inmates, and a previously determined cut-off score of $\mathbf{2 4}$ on the Keane Scale was confirmed as useful with this population. All three instruments, the CAPS-DX, PDSTM, and Keane Scale agreed on nine of 14 diagnoses for current PTSD. The current research confirmed previous research indicating that the number of potentially traumatic events experienced by an individual is associated with increased levels of PTSD symptomatology. Although it had been hypothesized that African-Americans and Hispanics would have higher rates of PTSD than Caucasians, just the opposite was found. Incarceration itself was identified as a traumatic stressor that can precipitate PTSD symptomatology. However, most of the identified PTSD cases were not the result of a discrete stressor, but of multiple stressors. The study's efficacy was limited by the sample's lack of representativeness of both the site and the federal inmate population as a whole. This resulted froin the unrepresentative racial composition of the research site and the diminished participation of AfricanAmerican inmates. This diminished participation may have resulted, in part, from the racial dissimilarity of the investigator. A study involving a greater number of participants, 
using researchers of varying racial backgrounds, may help confirm and expand upon the results of the current study. 


\section{Curriculum Vitae: Robert K. Guthrie}

\section{Current Placement}

Completing an American Psychological Association-approved, pre-doctoral internship at the Veterans Administration Medical Center in Salem, Virginia. Internship extends from Augusi 3i, 1998 to August 27, 1999.

\section{Current Education}

Pursuing a Ph.D. in Counseling Psychology at West Virginia University (WVU).

Estimated date of completion is August, 1999.

\section{Awards}

Arlen and Louise Stone Swiger Doctoral Fellowship, WVU, (1995-1998).

West Virginia Rural Health Initiative (RHI) Fellowship, 1996.

Outstanding RHI Community Service Project, 1996.

Professional Affiliations

American Psychological Association

International Society for Traumatic Stress Studies

\section{Professional Experience}

Psychology Intern, Federal Correctional Institution, Cumberiand, MD, August, 1996April, 1997

WV Rural Health Initiative Fellow, Practice of Dr. Thomas B. Stein, Moorefield and Petersburg, WV, Summer, 1996.

Psychology Intern, The Thomas B. Finan Center, Cumberland, MD, September, 1994April, 1995.

\section{Previous Education}

MA, Counseling, WVU, 1995.

Graduate economics studies, Universität Regensburg, Regensburg, Germany, 1971-1974.

German language certificate, Defense Language Institute, Washington, DC, 1969.

BA, History, Yale University, 1968.

\section{Military Service}

US Army, Military Intelligence, 1968-1971. 


\section{Other Work Experience}

International Marketing Consultant and Freelance Writer, 1993-95.

General Manager, HVB International Ltd., 1989-93.

Executive Director, National Foundation for Orthopaedics and Rehabilitation, 1988.

Director of Public Affairs, The Phillips Collection, 1983-87.

Director of Development, American Symphony Orchestra League, 1981-82.

Director of Development, National Symphony Orchestra, 1978-81.

Director of Development, American Heart Association of Northern Virginia and Washington, DC, 1976-78.

Federal Election Commission, Auditor/Investigator, 1975-76.

Economics and English instructor, Universität Regensburg, 1973-74. 


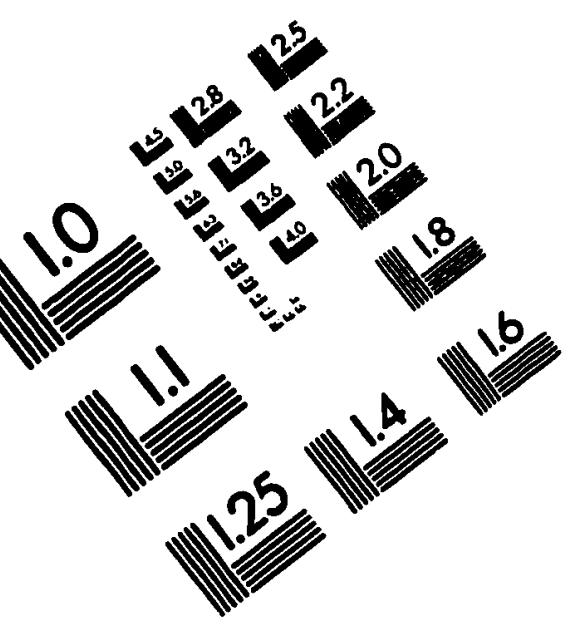
IMAGE EVALUATION
TEST TARGET (QA-3)
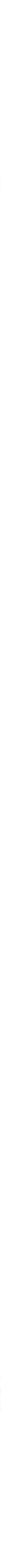